\title{
Development of a genetically encoded model for the sensing of glutathione redox potential in human embryonic stem cell-derived cardiomyocytes and fibroblasts
}

\section{Doctoral Thesis}

In partial fulfilment of the requirements for the degree

\author{
"Doctor rerum naturalium (Dr. rer. nat.)" \\ in the Molecular Medicine Study Program \\ at the Georg-August University Göttingen
}

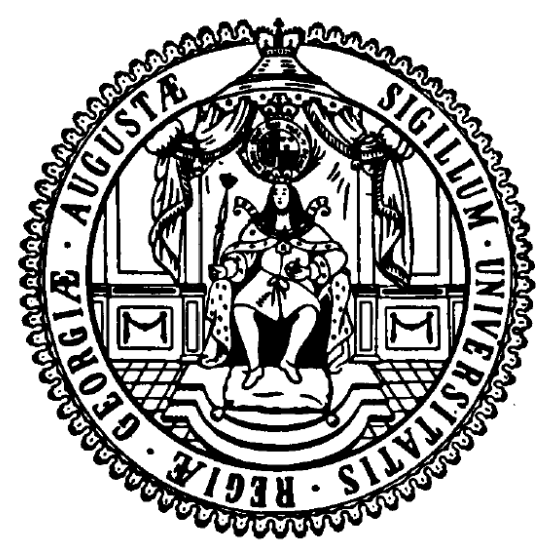

Submitted by

Eriona Heta

(born in Shkoder, Albania)

Göttingen 2017 


\section{Members of the Examination Board:}

Prof. Dr. med. Wolfram-Hubertus Zimmermann (Supervisor)

Email: w.zimmermann@med.uni-goettingen.de

Phone: $\quad$ +49 (0) 551395787

Postal Address: Institute of Pharmacology and Toxicology University Medical Center Goettingen Georg-August University Göttingen Robert-Koch-Str. 40 37075 Göttingen Germany

Prof. Dr. Viacheslav O. Nikolaev (Second member of thesis committee)

Email: $\quad$ v.nikolaev@uke.de

Phone: $\quad+49(0) 40741051391$

Postal Address: Institute of Experimental Cardiovascular Research University Medical Center Hamburg-Eppendorf (UKE) Martinistr.52 20246 Hamburg

Prof. Dr. mult. Thomas Meyer (Third member of thesis committee)

Email: thomas.meyer@medizin.uni-goettingen.de

Phone: $\quad+49(0)$ 0551-39-4881

Postal Address: Department of Molecular Psychocardiology

Waldweg 33

D- 37075 Göttingen

Germany

Prof. Dr. Manuel Mayr (Forth member of thesis committee)

Email: $\quad$ manuel.mayr@kcl.ac.uk

Phone: $\quad$ +44 (0) 2078485132

Postal Address: The James Black Centre King's College, University of London 125 Coldharbour Lane London SE5 9NU

United Kingdom

Date of Disputation: April $3^{\text {rd }}, 2017$ 


\section{AFFIDAVIT}

Here I declare that my doctoral thesis entitled:

"Development of a genetically encoded model for the sensing of glutathione redox potential in human embryonic stem cell-derived cardiomyocytes and fibroblasts"

has been written independently with no other sources and aids than quoted.

Eriona Heta

Göttingen, February 2017 
"I am among those who think that science has great beauty. $\mathcal{A}$ scientist in his laboratory is not only a technician: $\mathcal{H}$ is also a child placed before natural phenomena Which impress him like a fairy tale." 


\section{List of publications:}

Weber, M., Heta, E., Moreira, R., Gesche, V.N., Schermer, T., Frese, J., Jockenhoevel, S., and Mela, P. (2014). Tissue-engineered fibrin-based heart valve with a tubular leaflet design. Tissue Eng Part C Methods 20, 265-275.

\section{List of poster presentations:}

Eriona Heta, Poh Loong Soong, Angelica Roa, Manuel Mayr, Wolfram-Hubertus Zimmermann (2015). Assessment of reactive oxygen species in human embryonic stem cell derived cardiomyocytes and fibroblasts. BHF Center of Excellence annual symposium, London, UK.

Eriona Heta, Poh Loong Soong, Viacheslav Nikolaev, Manuel Mayr, WolframHubertus Zimmermann (2015). Reporting ROS in human cardiomyocytes and fibroblasts with the genetically encoded redox sensor Grx1-roGFP2.DGK Basic Science meeting 2015, Berlin, Germany.

Eriona Heta, Poh Loong Soong, Viacheslav Nikolaev, Dörthe Katschinski, Manuel Mayr, Wolfram-Hubertus Zimmermann (2016). Reporting redox states of glutathione in human cardiomyocytes and fibroblasts with the genetically encoded redox sensor Grx1-roGFP2.DGK Basic Science meeting 2016, Berlin, Germany. 


\section{$\underline{\text { Table of Contents }}$}

Acknowledgements ......................................................................... i

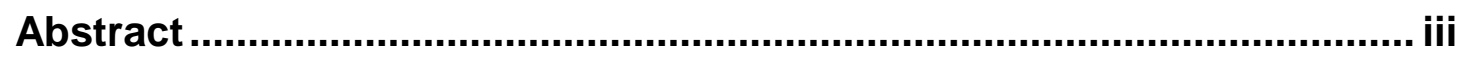

List of Figures .......................................................................................... iv

List of Tables ........................................................................................... vii

Abbreviations.................................................................................... viii

1. Introduction ................................................................................. 1

1.1 Cellular composition of the heart................................................. 1

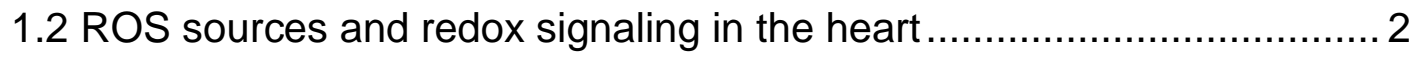

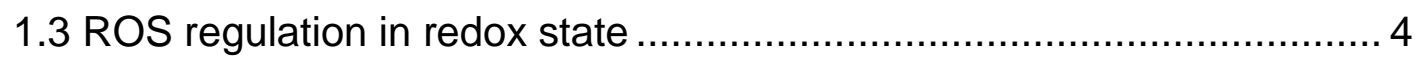

1.4 Measurements of ROS and redox states .................................. 8

1.4.1 Genetically modified fluorescent redox sensors ......................... 9

1.4.2 Mechanism of redox sensing with Grx1-roGFP2 …................ 10

1.5 Drug-ROS sensing and therapeutic opportunities ......................... 11

1.6 Engineered heart muscle as a 3D model to study redox changes ....... 14

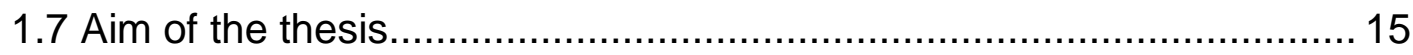

2. Materials and Methods ............................................................. 17

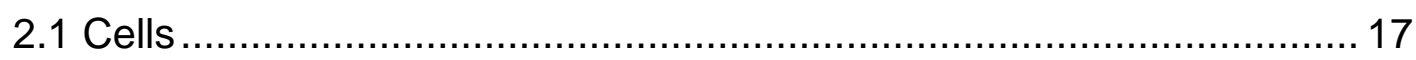

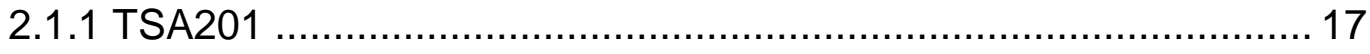

2.1.1.1 Cell Culture and maintenance of cell lines ......................... 17 
2.1.1.2 Freezing and thawing of TSA201 cells 17

2.1.2 Human foreskin fibroblasts 18

2.1.2.1 Culturing and passaging of HFFs 18

2.1.3 Human embryonic stem cells. 18

2.1.3.1 Monolayer differentiation of HES2-CM 18

2.1.3.2 Single cell dissociation of HES2-CM 19

2.1.3.3 Freezing and thawing of HES2-CM. 19

2.1.4 Flow cytometry 20

2.1.4.1 Live cell analysis 20

2.1.4.2 Fixed cell suspensions analysis 20

2.2 Cloning of pGIPZ- Grx1-roGFP2 21

2.2.1 Polymerase Chain Reaction (PCR) 23

2.2.2 Agarose gel electrophoresis 25

2.2.3 Plasmid DNA extraction from agarose gels . 25

2.2.4 Ligation. 25

2.2.5 Transformation 26

2.2.6 Miniplasmid DNA preparation 27

2.2.7 Restriction enzyme digestion. 28

2.2.8 Maxiplasmid DNA preparation 29

2.2.9 DNA sequencing. 30 
2.3 Transduction of Grx1-roGFP2 in HFFs and HES2-CM 30

2.3.1 Lentivirus production 30

2.3.2 Lentivirus purification 31

2.3.3 Lentivirus transduction of HFFs and HES2-CMs 32

2.4 Redox measurements of transgenic HFFs and HES2-CMs 33

2.4.1 Calculation of $E_{\text {roGFP2 }}$ redox potentials 35

2.5 Redox response to reference compounds 36

2.6 Oxidized peroxiredoxin in fibroblasts and cardiomyocyte 36

2.6.1 Protein isolation 36

2.6.2 Protein quantification via Bradford Assay 37

2.6.3 SDS-PAGE and immunoblotting analysis 37

2.7 Generation and analysis of Human Engineered Heart Muscle 39

2.7.1 Construction and casting of EHMs 39

2.7.2 Visualisation of redox changes in transgenic EHMs

2.7.3 Isometric force measurements 42

2.7.4 EHM dissociation 42

2.7.5 Immunofluorescent staining and low cytometry 43

2.8 Statistics 43

3. Results.

3.1 Cloning of pGIPZ- Grx1-roGFP2 44 
3.1.1 Integration of Xbal and Notl cutting sites on CMV-Grx1-roGFP2 .. 44

3.1.2 Generation of the lentivirus plasmid pGIPZ-Grx1-roGFP2 45

3.2 Grx1-roGFP2 expression in TSA cells and lentivirus production. 47

3.2.1 Lentivirus transduction of HFFs and HES2-CM 48

3.3 Monolayer glutathione redox measurements 50

3.3.1 Oxidative challenge with $\mathrm{H}_{2} \mathrm{O}_{2}$ 51

3.3.2 Response of HFF and HES2-CM to DA 53

3.3.3 Response of HFF and HES2-CM to DTT 55

3.3.4 Calculation of the glutathione redox potential 57

3.3.5 Glutathione redox changes upon cumulative redox challenge ......58

3.4 Grx1-roGFP2 responses to angiotensin II and inotropes 59

3.5 Detection of peroxiredoxin oxidation 64

3.6 Redox sensing in engineered heart muscle 65

3.6.1 Glutathione redox measurements in EHM. 65

3.6.2 Contraction force measurement 67

4. Discussion 69

4.1 Tools for redox quantification in living cells 69

4.2 Cell type specific glutathione redox responses 71

4.3 Cell type specific redox responses to angiotensin II and drugs. 73

4.4 Redox sensing in a heart muscle tissue context 74 


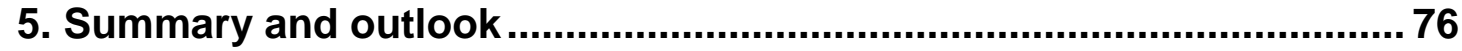

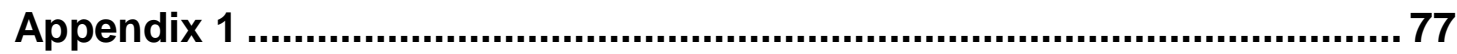

Appendix 2 ...................................................................................... 91

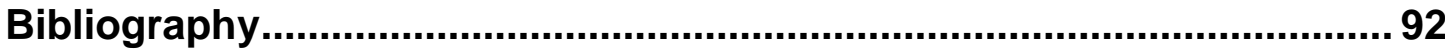




\section{Acknowledgements}

I would like to start with a big thank to everyone, who contributed in some way to my study and encouraged me during these years of PhD.

First of all, I would like to express my special thanks of gratitude to my supervisor Prof. Dr. Wolfram-Hubertus Zimmermann. It has been a great honor and pleasure to work under his supervision. I very much appreciate him for giving me the chance to be his representative student in IRTG 1816. I especially express my sincere thanks for his constant support, advice, freedom for experimental ideas and for his guidance throughout my doctoral studies.

Furthermore, I would like to express my genuine thanks to Prof. Dr. Viacheslav Nikolaev for his continuous assistance, advice, suggestions and motivation during these years. Special thanks go to Prof. Dr. Thomas Meyer for his support, suggestions and endless positive energy he provided at every discussion. I would like to thank Prof. Dr. Manuel Mayr for his appreciative ideas and suggestions during these years.

Many thanks go to Prof. Dr. Dörthe Katschinski for offering me her laboratory facilities for my study and for her insightful suggestions and advices during these years. I am very glad to have known so many supportive and friendly group leaders that always encourage their students.

My very special thanks go for Dr. Poh Loong Soong for all the support, knowledge and encouragement that he provided during these years. He has been a great friend and teacher. I thank him a lot for teaching me how to question thoughts and overcome insecurities, and how to become a good and independent scientist. My sincere thanks go to my kindest friend and colleague ever, Dr. Elif Levent. Her care, support and suggestions have always been appreciated in every situation. My genuine thanks go for my great colleagues and friends Dr. Sumon Sur, Dr. Satish Galla and Dr. Farah Raad for the admired encouragement and support during every circumstance. My thanks go to all the other members of the Institute of Pharmacology 
and Toxicology for the cheerful and friendly atmosphere that they provide. It has been a great pleasure to be part of this group.

I am very lucky to be surrounded by amazing people that give me lots of unconditional love and support in every situation. Many thanks to Dr. Genada Sinani and to many other close friends and relatives that I could not mention here. Thank you all for your kindness and for always being there for me.

Finally, my endless thanks go to my wonderful family who I dedicate my thesis. My parents, my brother, my sister-in-law and my lovely nephew (Ergin) and niece (AliaAdora), who have always been a fountain of love, care, inspiration, strength and encouragement through my entire life. This thesis would not be possible without their support. 


\section{Abstract}

Redox alterations due to increased ROS production in heart cells have been implicated in several cardiovascular diseases, such as ischemia, hypertrophy, and myocardial infarction, but may also play a crucial role for the wanted and unwanted effects of pharmacological stimulation. Genetically encoded biosensors allow for the visualization of redox changes at real-time in a quantitative manner, but have not yet been applied to human heart models. In this study, the hypothesis was tested that the cytosolic glutathione (GSH) redox sensor Grx1-roGFP2 can be applied to record the GSH redox state in human cardiomyocytes and fibroblasts. The cytosolic glutathione redox sensor Grx1-roGFP2 was stably introduced into human embryonic stem cell-derived cardiomyocytes (HES2-CM) and human foreskin fibroblasts (HFF) via lentiviral transduction. The kinetics of the Grx1-roGFP2 sensor were determined in a cell type specific manner in HES2-CM and HFF under increasing concentrations of $\mathrm{H}_{2} \mathrm{O}_{2}(0.1-1,000 \mu \mathrm{mol} / \mathrm{L})$, diamide $(1-1,000 \mu \mathrm{mol} / \mathrm{L})$, and DTT $(0.1-1 \mathrm{mmol} / \mathrm{L})$. This identified a more reduced $\mathrm{E}_{\mathrm{GSH}}$ in HES2-CMs (-289 $\left.\pm 1 \mathrm{mV} ; \mathrm{n}=56\right)$ compared to HFFs $(-269 \pm 2 \mathrm{mV} ; \mathrm{n}=18)$. Subsequently, GSH redox state alterations were investigated upon ROS stimulation with angiotensin II and pharmacological stimulation with cardioactive reference compounds (levosimendan, omecamtiv mecarbil). To investigate cell specific GSH redox changes in a tissue context, engineered heart muscles (EHM) were generated by mixing either (i) Grx1-roGFP2HFFs and naive HES2-CMs or (ii) Grx1-roGFP2 HES2-CMs and naive HFFs in a collagen hydrogel. Force generating EHM were formed within 20 days and were subjected to an acute oxidative $\left(\mathrm{H}_{2} \mathrm{O}_{2}\right)$ and reductive (DTT) challenge. GSH oxidation and reduction could be recorded optically and associated with a reduced and increased contractile performance in EHM. Taken together, GSH redox state can be recorded optogenetically in living human cardiomyocytes and fibroblasts in classical monolayer and $\mathrm{EHM}$ culture. Differences in $\mathrm{E}_{\mathrm{GSH}}$ suggest difference in redox related signalling in cardiomyocytes and fibroblasts which may have to be considered when interpreting redox responses to drugs or other stimuli at the whole heart level. 


\section{List of Figures}

Figure 1: Sources of ROS and the consequences of high and low ROS production. . 2

Figure 2: ROS pathways associated with cardiac hypertrophy and remodeling 4

Figure 3: ROS effects on glutathione and thioredoxin 6

Figure 4: Peroxiredoxin redox regulation 8

Figure 5: Schematic view of Grx1-roGFP2 sensor in oxidized and reduced conditions

Figure 6: Redox signaling pathway via Angll stimulation 14

Figure 7: Overview of the strategy, methods, and aims 16

Figure 8: Schematic overview of the cloning strategy 22

Figure 9: The assembled unit for lentivirus purification 32

Figure 10: Schematics of the fluorescence capture system used to measure redox changes in monolayer and tissue 34

Figure 11: EHM mold and stretcher construct design 39

Figure 12: EHM condensation and mechanical (dynamic) loading 41

Figure 13: Schematic view of the subcloning strategy....... 45

Figure 14: Verification of pGIPZ-CMV-Grx1-roGFP2 cloning 46

Figure 15: TSA cells after 72 hrs of transfection.

Figure 16: HFFs after transduction 48

Figure 17: Transduction efficiency in HFFs 49 
Figure 18: Transduction efficiency in HES2-CMs 50

Figure 19: Cell-specific analysis of the glutathione redox sensor in HFF and HES2$\mathrm{CM}$

Figure 20: Live imaging of glutathione oxidation in $\mathrm{HFF}$ under $\mathrm{H}_{2} \mathrm{O}_{2}$ challenge ........ 52

Figure 21: Live imaging of glutathione oxidation in HES2-CM under $\mathrm{H}_{2} \mathrm{O}_{2}$ challenge 52

Figure 22: Differences in glutathione oxidation kinetics in HFF and HES2-CM. 53

Figure 23: Live imaging of glutathione oxidation in HFF under DA challenge. 54

Figure 24: Live imaging of glutathione oxidation in HES2-CM under DA challenge . 54

Figure 25: Differences in biosensor oxidation kinetics in HFF and HES2-CM 55

Figure 26: Live imaging of glutathione oxidation in HFF under DTT challenge. 56

Figure 27: Live imaging of glutathione oxidation in HES2-CMs under DTT challenge. 56

Figure 28: Differences in glutathione reduction kinetics in HFF and HES2-CM. 57

Figure 29: Assessment of the dynamic range of the Grx1-roGFP2 sensor 58

Figure 30: Visualization of glutathione redox states under angiotensin II stimulation in HFF. 59

Figure 31: Visualization of glutathione redox states under angiotensin II stimulation in HES2-CM. 60

Figure 32: Visualization of glutathione redox states under levosimendan treatment in HFF. 61

Figure 33: Visualization of glutathione redox states under levosimendan treatment in HES2-CM. 62 
Figure 34: Visualization of glutathione redox states under omecamtiv mecarbil treatment in HFF. 63

Figure 35: Visualization of glutathione redox states under omecamtiv mecarbil 63

Figure 36: Confirmation of peroxiredoxin oxidation in HFF and HES2-CM upon $\mathrm{H}_{2} \mathrm{O}_{2}$ exposure 64

Figure 37: Expression and monitoring of the glutathione redox sensor in EHM. 65

Figure 38: Cell type specific visualization of redox alteration in engineered human myocardium 66

Figure 39: Assessment of potential Grx1-roGFP2 toxicity in EHM 67

Figure 40: Functional consequences of oxidation and reduction in EHM contractility. 68 


\section{List of Tables}

Table 1: Forward and reverse primers used for PCR amplification of CMV-Grx1roGFP2 23

Table 2: Components and parameters needed for PCR 24

Table 3: PCR protocol 24

Table 4: Ligation of insert and vector. 26

Table 5: Restriction enzyme digestion. 28

Table 6: DNA sequencing reaction mixture. 30

Table 7: Transfection reaction mixture for lentivirus production. 31

Table 8: List of primary/secondary antibodies and fluorescent conjugates. 38

Table 9: EHM master mix components 40

Table 10: $\mathrm{E}_{\mathrm{GSH}}$ of the glutathione sensor in HFF and HES2-CM 58

Table 11: Summary of Grx1-roGFP2 sensor activites in HES2-CM and HFF. 72

Table 12: Cell lines 91 


\section{Abbreviations}

aa

AA

ACE

Ang II

ANOVA

Amp

ARB

ATP

$A Z T$

$\mathrm{BH} 4$

bp

BSA

CaMKII

cDNA

CF

CM

$\mathrm{CO}_{2}$

CMV

c-Src

CTNI

Cx

CYS $_{P}$

CYS $_{R}$

DA

DMEM

DMF

DMSO

DNA

DNase

dNTP

Dox
Amino acid

Ascorbic Acid

Angiotensin Converting Enzyme

Angiotensin II

Analysis of variance

Ampicillin

Angiotensin Receptor Blockers

Adenine triphosphate

Azidothymidine

Tetrahydrobiopterin

Base pair

Bovine Serum Albumin

$\mathrm{Ca} /$ calmodulin-dependent kinase II

complimentary DNA

Cardiac fibroblast

Cardiomyocyte

Carbon Dioxide

Cytomegalovirus

Proto-oncogene tyrosine-protein kinase Src

Cardiac troponin I

Connexin

Peroxidatic cysteine

Resolving cysteine

Diamide

Dulbecco's Modified Eagle's medium

Dimethyl fumarate

Dimethyl sulfoxide

Deoxyribonucleic acid

Deoxyribonuclease

Deoxyribonucleotide triphosphate

Doxorubicin 

DTT
Dithiothreitol
ECC
Excitation-contraction coupling
ECM
Extracellular Matrix
EDTA
Ethylenediaminetetraacetic acid
EGFP
Enhanced green fluorescence protein
$\mathrm{E}_{\mathrm{GSH}}$
Reduction potential of glutathione
EHM
Engineered Heart Muscle
ER
Endoplasmic Reticulum
ESC
Embryonic stem cell
ESIPT
Excited-state intramolecular proton transfer
ET-1
Endothelin-1
$\mathrm{EtBr}$
Ethidium Bromide
ETC
Electron transport chain
$\mathrm{EtOH}$
Ethanol
$\mathrm{F}$
Forward primer
FACS
Fluorescence-activating cell sorting
FB
Fibroblast
FBS
Fetal Bovine Serum
FGF-2
Fibroblast Growth Factor-2
FITC-A
Fluorescein isothiocyanatefluorescence signal area
FN
Fibronectin
FRET
Fluorescence resonance energy transfer
FSC-A
Forward Scatter Area
g
Gravitational force
GAG
Glycosaminoglycan galactopyranoside
GAPDH
Glyceraldehyde-3-Phosphate Dehydrogenase
GFP
Green fluorescent protein
Grx1
Glutaredoxin 1
GSH
Reduced Glutathione
GSSG
Oxidized glutathione
$\mathrm{H}_{2} \mathrm{O}$
Water
$\mathrm{H}_{2} \mathrm{O}_{2}$
Hydrogen peroxide
HEPES
4-(2-hydroxyethyl)-1-piperazineethanesulfonic 
HES2-CM

HFF

HPQ

IGF1

$\lg G$

ILF2

IMDM

IPTG

L

LEVO

LIF

M

MAO

MAPK

MI

MMP

MPO

$\mathrm{NaCl}$

$\mathrm{NADH}$

$\mathrm{NaOH}$

NEAA

NEM

NeoR

NF-kB

NHE

NOS

NOX

Nrf2

OME

ONOO

OxD

$\mathrm{P} / \mathrm{S}$

PBS
Human Embryonic Stem Cell line 2 derived

Human foreskin fibroblasts

2-(2'-hydroxyphenyl)-4(3H)-quinazolinone

Insulin-like growth factor 1

Immunoglobulin $\mathrm{G}$

Interleukin Enhancer Binding Factor 2

Iscove's modified Dulbecco's medium

Isopropyl $\beta$-D-1-thiogalactopyranoside

Litre

Levosimendan

Leukemia inhibitory factor

Molar

Monoamine oxidases

Mitogen-activated protein kinase

Myocardial infarction

Matrix metalloproteinase

Myeloperoxidase enzyme

Sodium Chloride

Nicotinamide adenine dinucleotide

Sodium Hydroxide

Non-Essential Amino Acids

$\mathrm{N}$-Ethylmaleimide

Neomycin-Resistance

Nuclear factor-kB

$\mathrm{Na}^{+}-\mathrm{H}^{+}$exchanger

NO synthase

NADPH oxidase

Nuclear factor(erythroid derived)-like 2

Omecamtivmecarbil or (CK-1827452)

Peroxynitrite

Degree of oxidation

Penicillin/Streptomycin

Phosphate Buffered Saline 


\begin{tabular}{|c|c|}
\hline PCR & Polymerase Chain Reaction \\
\hline Phox & Phagocytic oxydase \\
\hline PKC & Protein kinase $\mathrm{C}$ \\
\hline PMN & Polymorphonuclear leukocyte \\
\hline Puro & Puromycin \\
\hline PVDF & Polyvinylidene Difluoride \\
\hline $\mathrm{R}$ & Reverse primer \\
\hline RAS & Renin-angiotensin system \\
\hline REDOX & Reduction-oxidation reaction \\
\hline roGFP2 & Reduction/oxidation sensitive GFP \\
\hline ROS & Reactive oxygen species \\
\hline RPMI & Roswell Park Memorial Institute \\
\hline RT & Room Temperature \\
\hline RYR & Ryanodine Receptor \\
\hline S & Nucleophilic sulphur \\
\hline SDS & Sodium Dodecyl Sulfate \\
\hline PAGE & Polyacrylamide Gel Electrophoresis \\
\hline SEM & Standard error of the mean \\
\hline SERCA & Sarcoplasmic reticulum $\mathrm{Ca}^{2+}$ ATPase pump \\
\hline SR & Sarcoplasmic reticulum \\
\hline SSC & Side-scattered light \\
\hline SV40 & Simian virus 40 \\
\hline Taq & Thermusaquaticus \\
\hline TBST & Tris-Buffered Saline and Tween 20 \\
\hline TGF- $\beta$ & Transforming Growth Factor beta \\
\hline TPFM & Two-photon fluorescence microscopy \\
\hline Trx & Thioredoxin \\
\hline$U$ & Unit \\
\hline V & Volts \\
\hline VEGF A & Vascular endothelial growth factor $A$ \\
\hline WT & Wild type \\
\hline$X G A L$ & 5-bromo-4-chloro-3-indolyl- $\beta$-D- \\
\hline XO & Xanthine oxidase \\
\hline
\end{tabular}


Abbreviations

YFP

$\alpha-\mathrm{MHC}$
Yellow fluorescence protein

Myosin heavy chain, Alpha isoform 


\section{Introduction}

\subsection{Cellular composition of the heart}

The human heart is a beating organ that consists mainly of cardiomyocytes, fibroblasts, endothelial cells, smooth muscle cells, and immune cells (Souders et al. 2009). Cardiomyocytes (CM) comprise almost $30 \%$ of the total cell pool while the remainder $70 \%$ are non-myocytes (Tirziu et al. 2010) with the cardiac fibroblasts (CF) being the most abundant cell type (Souders et al. 2009, Tian and Morrisey 2012). To understand the functional mechanics of the heart, it is essential to understand the biology of interactions of these cells.

Cardiomyocytes are contracting, striated and rod-shaped cells, which determine the performance of the heart. This energy dependent process depends critically on the provision of ATP produced in mitochondria. Actin and myosin filaments comprise the molecular machines that help the cell to contract. Furthermore, cardiomyocytes interact with their myocardial environment not only mechanically, but also by the secretion of cytokines and growth factors (Howard and Baudino 2014).

Cardiac fibroblasts, similarly to cardiomyocytes, are of mesodermal origin and considered the main contributor to extracellular matrix (ECM) of the heart (Souders et al. 2009). In addition, they are capable of sensing and responding to biochemical stress by secreting cytokines and growth factors (MacKenna et al. 2000). The secretion of several factors such as Ang II, IGF-1, transforming growth factor-B1 (TGF-ß), endothelin-1 (ET-1) and FGF2 (Bouzegrhane and Thibault 2002, Bujak and Frangogiannis 2007, Manabe et al. 2002) can influence cardiomyocyte contractility, metabolism, and oxygenation (Tirziu et al. 2010, Tomasek et al. 2002). In the recent years, the role of cardiac fibroblasts for cardiac physiology and pathology has been increasingly considered (MacKenna et al. 2000, Souders et al. 2009, Tiburcy and Zimmermann 2014). 


\subsection{ROS sources and redox signaling in the heart}

The beating heart requires ATP which for the most part (95\%) is provided by oxidative phosphorylation in mitochondria and for a minor part by the glycolysis and the Krebs cycle; $70-90 \%$ is produced by oxidation of fatty acids, while $10-30 \%$ is produced by oxidation of glucose, lactate, ketone bodies, and amino acids (Doenst et al. 2013). A byproduct of oxidative phosphorylation is the generation of ROS. ROS are highly reactive free radical molecules containing oxygen $\left(\mathrm{O}_{2^{-}},-\mathrm{OH}, \mathrm{H}_{2} \mathrm{O}_{2}\right)$, which are implicated in a myriad of cellular pathways, defined as redox signaling (Burgoyne et al. 2012). In addition to the ROS production by the mitochondrial electron transport chain (ETC), there are several dedicated enzymes, such as the nicotinamide adenine dinucleotide phosphate (NADPH) oxidases (NOX), NO synthases (NOS), xanthine oxidase (XO), and monoamine oxidases (MAO) that contribute to overall cellular ROS load (Burgoyne et al. 2012, Santos et al. 2016). A delicate interplay of ROS and antioxidants control the level of cellular oxidation and reduction and thus a number of biological processes (Figure 1).

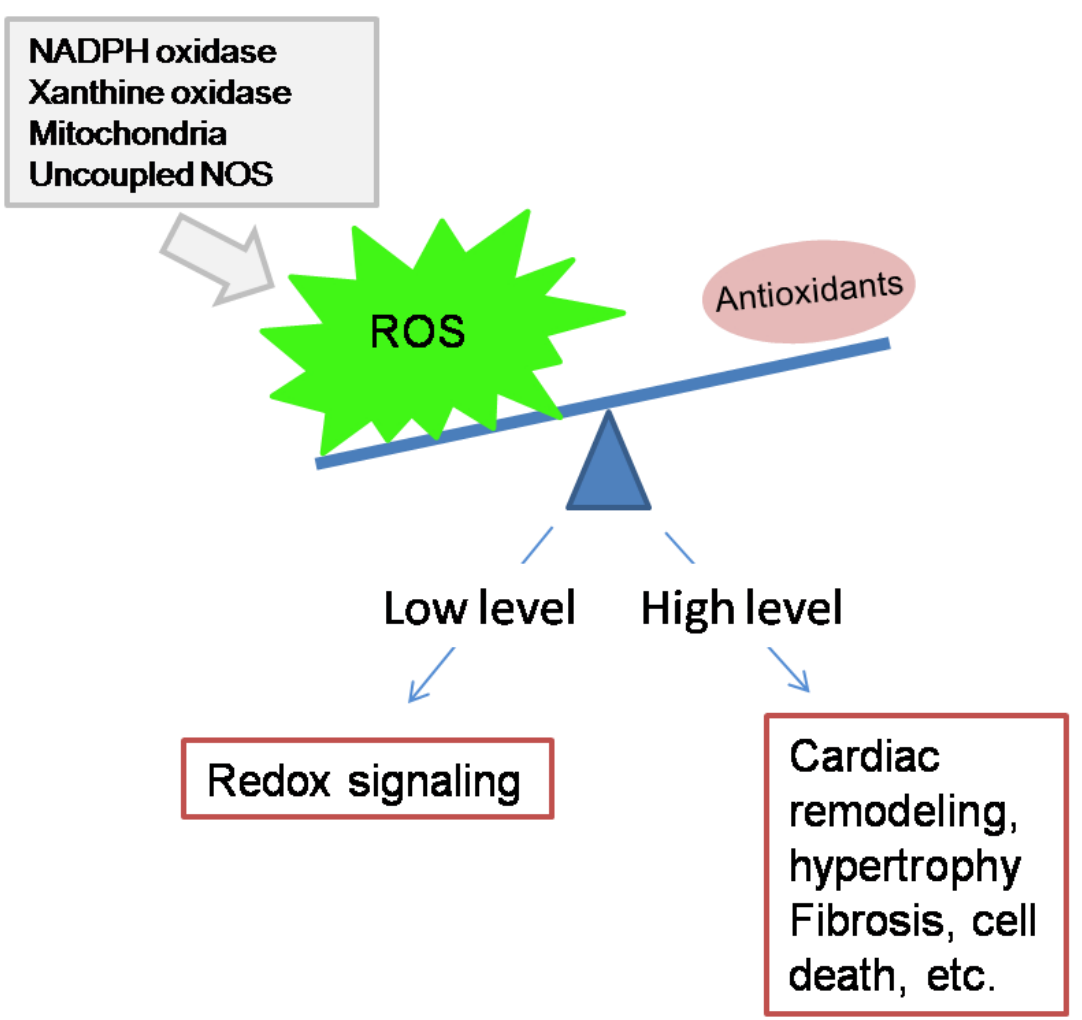

Figure 1: Sources of ROS and the consequences of high and low ROS production. Image adapted from (Tsutsui et al. 2011). 
Mitochondrial ROS is produced mainly by complexes I and III of the ETC (Doenst et al. 2013). MAOs generate $\mathrm{H}_{2} \mathrm{O}_{2}$ during catabolic reactions and $\mathrm{XOs}$ generate $\mathrm{H}_{2} \mathrm{O}_{2}$ or $\mathrm{O}_{2}{ }^{-}$by using oxygen as an electron acceptor. NOS enzymes require tetrahydrobiopterin $(\mathrm{BH} 4)$ as a co-factor for $\mathrm{NO}$ production. The lack of $\mathrm{BH} 4$ leads to enhanced $\mathrm{O}_{2}^{-}$production which reacts with $\mathrm{NO}$ to $\mathrm{ONOO}^{-}$; peroxynitrite that leads to a further reduction in $\mathrm{BH} 4$ and can induce cell death by apoptosis and necrosis (Burgoyne et al. 2012, Chen et al. 2010). Finally, NOX enzymes are considered key players in cardiac ROS production. In human, this family of enzymes consists of seven isoforms (NOX 1-5 and dual oxidase 1-2), which require distinct subunits for their catalytic reactions (Lassegue et al. 2012, Santos et al. 2016). NOX 2 and NOX 4 are respectively considered the main $\mathrm{O}_{2}{ }^{-}$and $\mathrm{H}_{2} \mathrm{O}_{2}$ generating isoforms in the heart (Burgoyne et al. 2012, Hafstad et al. 2013, Lassegue et al. 2012, Sirker et al. 2011). NOX 2 is reported to be activated by angiotensin II (Angll), endothelin-1, growth factors, cytokines or mechanical forces. In contrast, NOX 4 is constitutively active; stimuli, such as hypoxia, endoplasmic reticulum (ER) stress, TGF- $\beta$ and ischemia have however shown to increase NOX 4 protein abundance (Burgoyne et al. 2012).

Studies show that physiological amounts of ROS are necessary for cell survival, proliferation and function (Figure 1). Several signaling pathways are directly influenced by ROS (Figure 2) and its excess production and accumulation can damage a number of cellular/sub-cellular components such as membranes, proteins and DNA (Charles and Eaton 2008, Elahi et al. 2009, Handy and Loscalzo 2016, Sabri et al. 2003, Sarsour et al. 2009, Sawyer et al. 2002, von Harsdorf et al. 1999). Examples of ROS mediated regulation of cellular processes include the $\mathrm{Na}^{+}-\mathrm{H}^{+}$ exchanger (NHE) and mitogen-activated protein kinases (MAPK) (Sabri et al. 1998) as well as tyrosine kinase Src pathways, protein kinase C, GTP-binding RAS proteins (Amin et al. 2001, Nakamura et al. 1998, Takimoto and Kass 2007). Specifically in cardiomyocytes, high ROS contributes to cell dysfunction and cardiac remodeling (Cesselli et al. 2001) by stimulation of apoptosis signaling kinase-1 (Kwon et al. 2003), activation of nuclear factor KB (NFKB) (Takimoto and Kass 2007), and reduction of $\mathrm{Ca}^{2+}$ uptake by SR $\mathrm{Ca}^{2+}$ ATPase (SERCA) (Xu et al. 1997). In addition, ROS inhibits the L-type $\mathrm{Ca}^{2+}$ current (Fearon et al. 1999) and affects the release of sarcoplasmic reticulum (SR) $\mathrm{Ca}^{2+}$ as a result of ROS modification of the cysteine 
sulphydryl groups of the ryanodine receptor (RyR) (Kawakami and Okabe 1998, Zima and Blatter 2006). Effects of ROS in cardiac fibroblasts are reported to involve: (1) activation of matrix metalloproteinases (MMP), which leads to fibrosis and cardiac remodeling (Siwik et al. 2001, Sorescu and Griendling 2002, Spinale 2002) and (2) induced CF proliferation (Cheng et al. 2003, Takimoto and Kass 2007). Accordingly, elevation of ROS is implicated in myocardial infarction, fibrosis, atherosclerosis, cardiac hypertrophy, ischemia-reperfusion, and heart failure (Bolli 1998, Giordano 2005, Tsutsui et al. 2011).

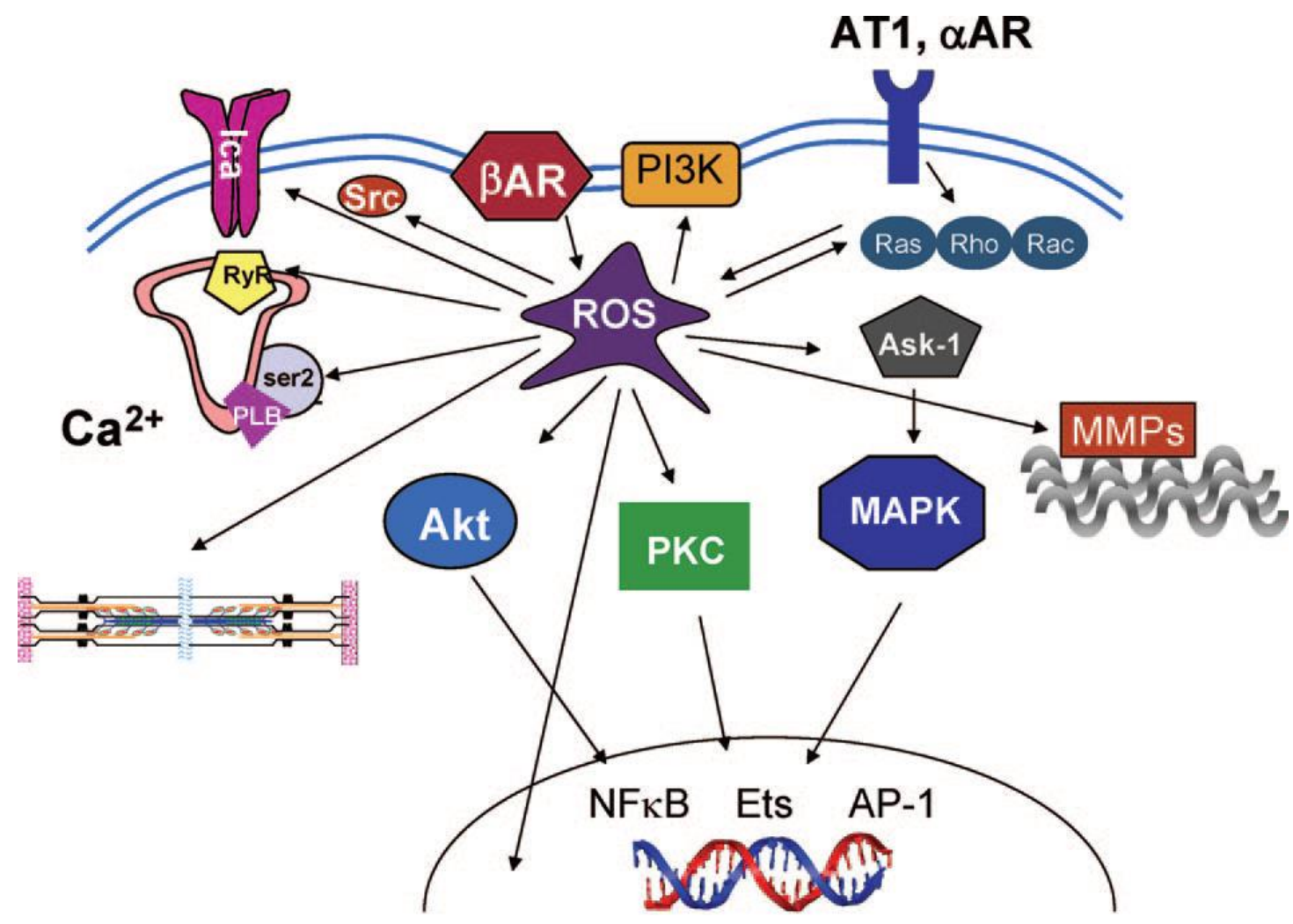

Figure 2: ROS pathways associated with cardiac hypertrophy and remodeling. Image taken from (Takimoto and Kass 2007).

\subsection{ROS regulation in redox state}

The term 'redox state' is linked with the redox equilibrium of redox couples inside the cell, such as reduced and oxidized glutathione (GSH/GSSG), thioredoxin (Trx/TrxSS) and other cysteine (Cys/CySS) containing proteins. The most abundant cellular antioxidant enzymes include glutathione, thioredoxin, and peroxiredoxin, which play 
an important role in cellular redox balancing and regulation (Hafstad et al. 2013, Kang et al. 2005). Nicotinamide adenine dinucleotide phosphate (NADPH) is the main source of reducing equivalents of the GSH and Trx proteins (Schafer and Buettner 2001). GSH/GSSG is considered the main cellular redox system and found in high amounts in most cell compartments (cytosol, mitochondria, ER and nucleus) (Mari et al. 2010, Schafer and Buettner 2001). In the cytosol, glutathione abundance has been reported to be within the range of 2-10 mmol/L (Hwang et al. 1992, Mari et al. 2010). In the ER enhanced oxidation is reflected by a higher abundance of GSSG. Mitochondria contain about 15\% of the total glutathione pool (Mari et al. 2009, Ribas et al. 2014). The cellular and subcellular glutathione redox potential $\left(E_{G S H}\right)$ can be calculated using the Nernst equation, considering an equilibrium of $\mathrm{E}_{\mathrm{GSH}}$ and $\mathrm{E}_{\mathrm{roGFP} 2}$ (Meyer and Dick 2010, Morgan et al. 2011).

Basal levels and states of cellular ROS are altered by free radical scavengers such as superoxide dismutase (SOD), glutathione peroxidase and catalase. The balance of the constantly oscillating reduction and oxidation processes are described as the "redox state" of the cell (McCord 1988, Werns and Lucchesi 1989).

In comparison to other ROS, $\mathrm{H}_{2} \mathrm{O}_{2}$ is the most stable ROS species and the mitochondria are the main compartments for $\mathrm{H}_{2} \mathrm{O}_{2}$ production. $\mathrm{H}_{2} \mathrm{O}_{2}$ is essential in intracellular redox signaling, signal transduction and plays a role in oxidative modifications of the sulfhydryl (thiol) group (-SH) of cysteines at the active site of enzymatically active proteins. Mammalian cells developed self-protective mechanisms to regulate and to protect themselves from the damaging $\mathrm{H}_{2} \mathrm{O}_{2}$ levels. This self-protecting mechanism could be an enzymatic or non-enzymatic system (Bienert et al. 2006, Malinouski et al. 2011, Veal et al. 2007). Antioxidant proteins such as glutathione, thioredoxin and peroxiredoxin play key roles in scavenging ROS (Giles 2006, Tew and Townsend 2011, Zhang and Martin 2014).

When ROS is present in the cellular system, due to enzymatic activities or exogenous stimulation (Figure 3), SOD mediates the conversion of superoxide to $\mathrm{H}_{2} \mathrm{O}_{2}$. In the presence of $\mathrm{H}_{2} \mathrm{O}_{2}$, the stimulation of the glutathione system is facilitated by the activation of glutathione peroxidase (GPx) (Liu et al. 2004) that mediates 
oxidation of GSH to GSSG while detoxifying $\mathrm{H}_{2} \mathrm{O}_{2}$ into $\mathrm{H}_{2} \mathrm{O}$ (McCord et al. 1971, Weydert and Cullen 2010). The reverse reduction process is mediated by glutathione reductase (GR) that receives electrons from NADPH (Watson et al. 2003) and helps in the conversion of GSSG to GSH. Moreover, the GSH/GSSG ratio is itself affected by the redox state of the thioltransferase glutaredoxin system (Grx; Figure 3) (Yoshioka 2015).

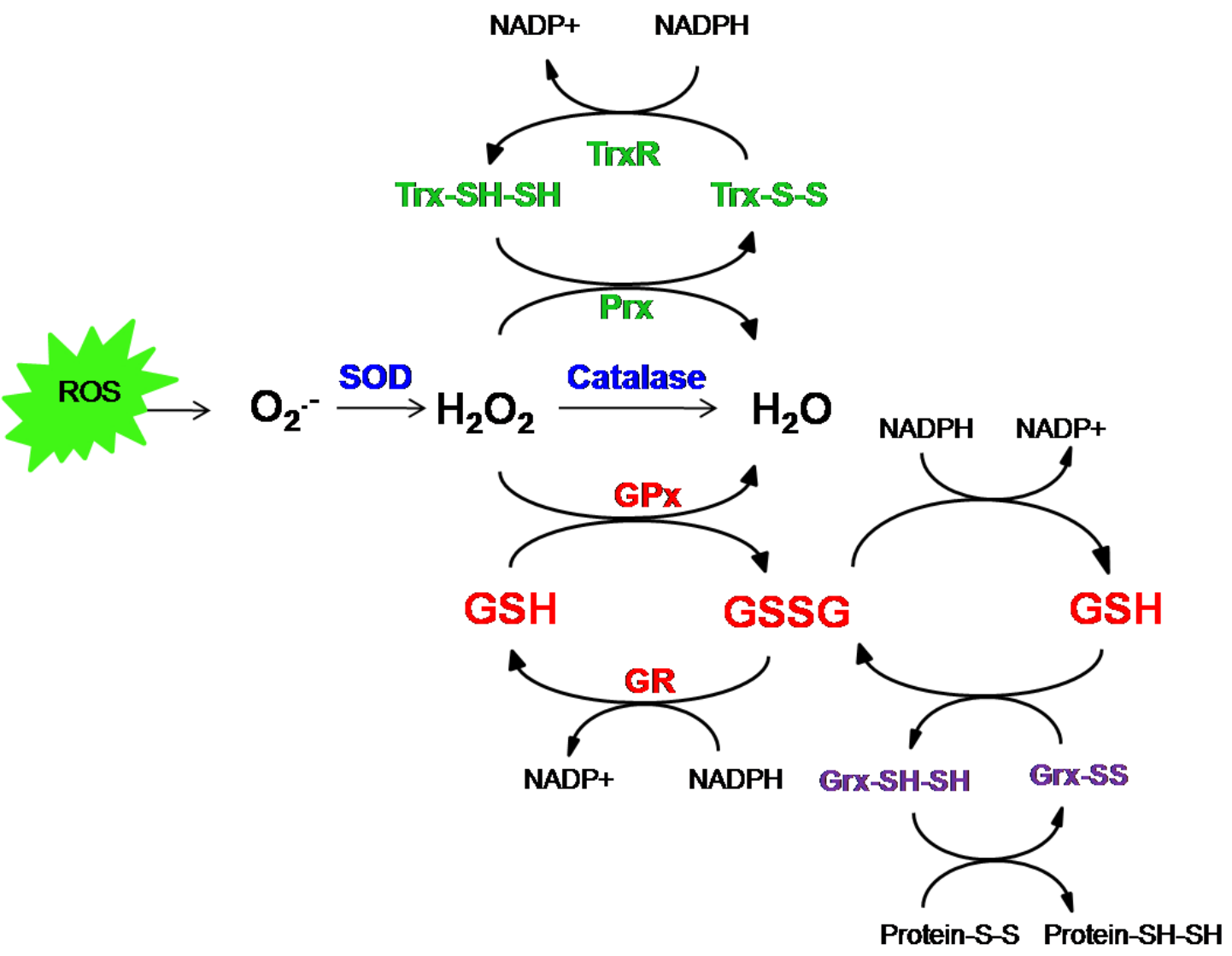

Figure 3: ROS effects on glutathione and thioredoxin. Oxidation leads to disulfide formation (-SS-) while reduction resolves the disulfides to thiol (-SH) residues in classical redox couples. Reduced and oxidized glutathione (GSH and GSSG) and thioredoxin (Trx-SH and Trx-SS) protein and its dependence on ROS, SOD and Catalase are depicted. GSH/GSSG state is further influenced by the redox state of the thioltransferase glutaredoxin (Grx) (Zhang and Martin 2014). 
Thioredoxin, specifically Trx1, is known for the specific cardioprotective function in the heart (Yoshioka 2015). The thioredoxin is protonated by oxidoreductases such as thioredoxin reductase (TrxR) and oxidized in the presence of peroxiredoxin (Prx) which at the same time is reduced (Figure 3) (Powis et al. 2000, Powis and Montfort 2001, Wood et al. 2003, Yoshioka 2015, Zhang and Martin 2014). Prx oxidation is typically analysed to determine the general state of oxidation in cells and tissue. The redox regulation of Prx is further detailed in Figure 4. Peroxiredoxins are important antioxidant enzymes and assist in maintaining low intracellular levels of $\mathrm{H}_{2} \mathrm{O}_{2}$. The 2Cys (Cys 51 and 172) peroxiredoxin enzymes are found in 4 different types (Prx 1,2,3 and 4) (Riquier et al. 2014). These proteins contain Cys with some nucleophilic sulphur (S) on their side chains that are very reactive to oxidation (Griendling et al. 2016, Nagahara et al. 2009).Formation of sulfenic $(-\mathrm{SOH})$, sulfinic $\left(-\mathrm{SO}_{2} \mathrm{H}\right)$ and sulfonic $\left(-\mathrm{SO}_{3} \mathrm{H}\right)$ derivatives (Figure 4$)$ due to oxidation lead to post-translational modifications of several proteins (Munns et al. 2005). 


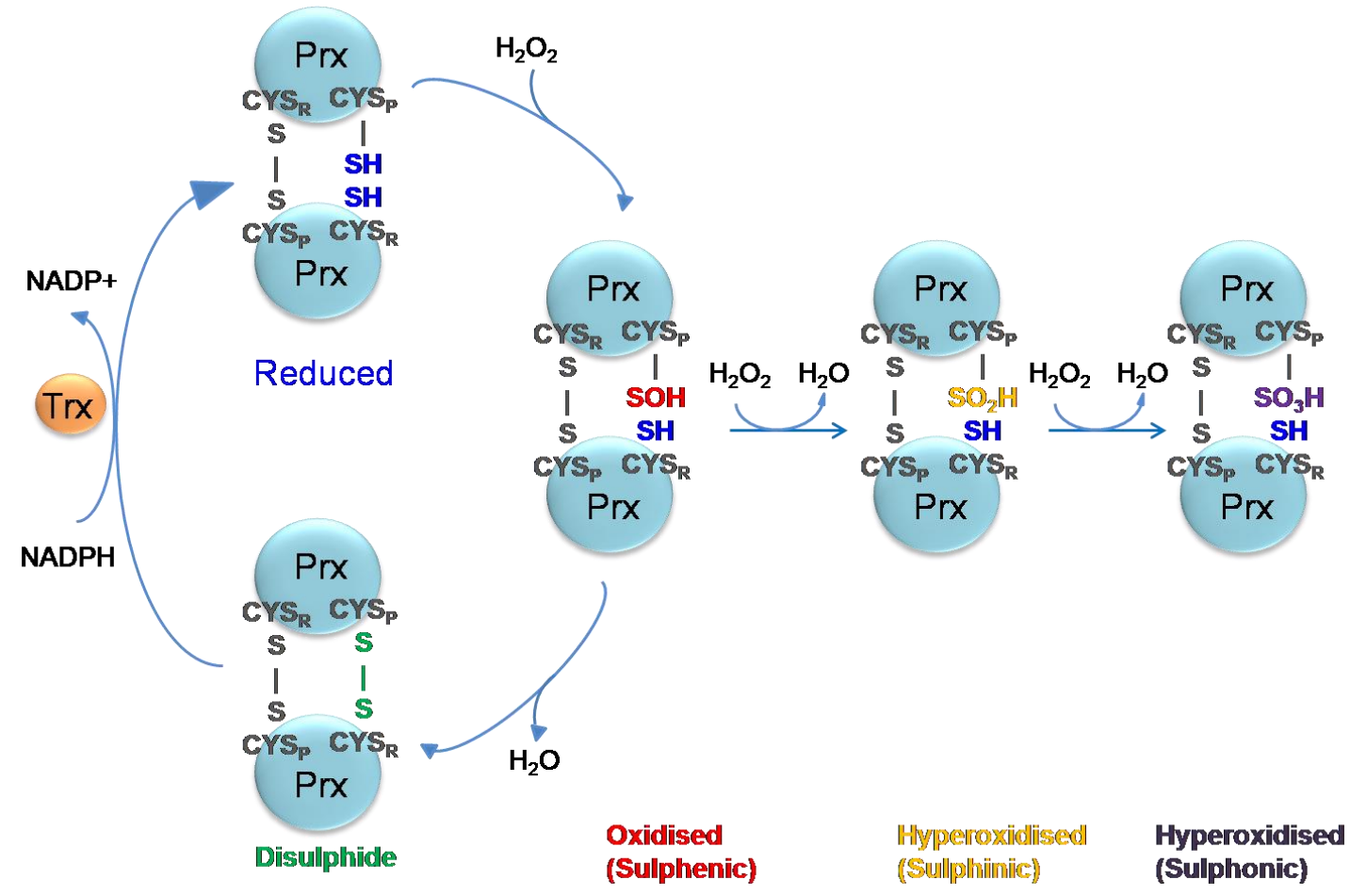

Figure 4: Peroxiredoxin redox regulation. Reduction of disulphides on peroxidatic $\left(C Y S_{P}\right)$ and resolving cyteines $\left(C Y S_{R}\right)$ of peroxiredoxin (Prx) dimers is mediated by thioredoxin (Trx). CYS is oxidized to sulphenic $(-\mathrm{SOH})$ residues and hyperoxidized to sulphinic $\left(-\mathrm{SO}_{2} \mathrm{H}\right)$ and sulphonic $\left(-\mathrm{SO}_{3} \mathrm{H}\right)$ residues, upon high $\mathrm{H}_{2} \mathrm{O}_{2}$ exposure. Image was adapted from (Hoyle and O'Neill 2015).

\subsection{Measurements of ROS and redox states}

Different strategies used to measure ROS and redox states in cells have been reviewed recently by Griendling et al (Griendling et al. 2016). Biochemical assays (e.g., dichlorodihydrofluorescein diacetate, amplex red, and dihydrorhodamine) have been routinely used to measure cellular redox changes. However, several limitations have been associated with such methods, including cell toxicity, oxidation artifacts, lack of dynamic measurements, and limited specificity (Forkink et al. 2010, Meyer and Dick 2010). This led to the development of optogenetic tools to study redox changes. 


\subsubsection{Genetically modified fluorescent redox sensors}

In recent years, genetically modified methods were developed to help monitor intracellular redox state changes in cells. Hydrogen peroxide sensor (Hyper) is a realtime, ratiometric sensor that is sensitive to $\mathrm{H}_{2} \mathrm{O}_{2}$. It is characterized by 2 excitation peaks at 410 and $500 \mathrm{~nm}$ and an emission at $519 \mathrm{~nm}$; its signal changes are reversible (Belousov et al. 2006) with a fast reaction constant (Bilan et al. 2013). As for most fluorescent-protein sensors, $\mathrm{pH}$ effects on signal intensity need to be considered (Lukyanov and Belousov 2014). Improvements of sensors are generally by enhancing redox-coupling, dynamic range and localization of sensors to subcellular compartments (Albrecht et al. 2011, Ezerina et al. 2014).

The first genetic redox sensing modifications were performed by adding cysteine residues at the $\mathrm{N} 149 \mathrm{C}$ and $\mathrm{S} 202 \mathrm{C}$ positions within the green fluorescent protein (GFP). Similar mutations were subsequently demonstrated to be effective in a redoxactive yellow fluorescence protein (rxYFP) (Ostergaard et al. 2001). When this modified sensor was subjected to redox processes, a spectral change occurred at $404 \mathrm{~nm}$ and $512 \mathrm{~nm}$ due to a shift in $\mathrm{H} 148$ and Y203 positions resulting from the formation of the disulfide bond between C149-C202 that faces the chromophore (Maulucci et al. 2008). This process allowed the visualization of the signal in real time and the changes in spectral levels could be used to report absolute concentrations of reduced and oxidized glutathione (GSH and GSSG, respectively) residues in cells (Ostergaard et al. 2004). However, to overcome the slow reaction of protein equilibrium due to differing glutathione states, the protein was fused with a recombinant glutaredoxin enzyme (Bjornberg et al. 2006). This model was further improved with another redox sensitive probe roGFP2 (Meyer and Dick 2010). In their study, Meyer and Dick introduced cysteine residues in S147 and Q204 positions of wild-type GFP (wtGFP) and enhanced GFP (EGFP). These new cysteine residues are located on B-strands 7 and 10, close to positions 148 and 204 facing the chromophore (Figure 5B). This allows the formation of intramolecular disulfide bridge between the two residues (Meyer and Dick 2010). The redox probe was named as roGFP1 (derived from wtGFP) when introducing C48S mutation, and roGFP2 (derived from EGFP) when adding another mutation S65T (Dooley et al. 2004, Hanson et al. 2004). In comparison to rxYFP, roGFPs are more sensitive to changes 
of $\mathrm{GSH} / \mathrm{GSSG}$ oxidation in a reduced environment due to their lower midpoint potential (-291 mV for roGFP1 and -280mV for roGFP2 (Dooley et al. 2004). Furthermore, they are ratiometric and have an increased signal to noise ratio or dynamic range, which is determined by the proportion of the maximum to minimum response of the excitation ratios (Hanson et al. 2004). Ratiometric analysis of roGFP probes are useful tools to visualize the oxidation and reduction equilibrium of glutathione/glutathione disulfide (GSH/GSSG) fluorescence ratio in real time at 400 $\mathrm{nm}$ and 490 nm (Dooley et al. 2004, Hanson et al. 2004, Meyer and Dick 2010). In this study, we used the Grx1-roGFP2 redox sensitive probe to measure the cytosolic glutathione potential.

\subsubsection{Mechanism of redox sensing with Grx1-roGFP2}

There are several advantages of utilizing roGFP2 relative to roGFP1, including enhanced brightness and dynamic range. The anionic form of the roGFP2 chromophore $(490 \mathrm{~nm})$ dominates over the protonated form $(405 \mathrm{~nm})$ at basal state, thus during oxidation due to the decrease in the anionic form and an increase in protonic form of the chromophore, a better signal could be obtained (Hanson et al. 2004). In roGFP2, the mutation S65T adds further resistance to artifacts such as photoswitching (Meyer and Dick 2010). roGFP2 was fused to human glutaredoxin redox enzyme Grx1 to accelerate the thiol-disulfide exchange between roGFP molecules and glutathione (Gutscher et al. 2008). Grx1-roGFP2 was reportedly very sensitive to traces of GSSG in the reduced environment of the cytosol, as a result of the redox potential $\left(E_{G S H}\right.$ ) ranging between $-240 \mathrm{mV}$ and $-320 \mathrm{mV}$ (Lukyanov and Belousov 2014). The reversibility of the Grx1-roGFP2 biosensor signal includes three consecutive steps (Figure 5A). Oxidized glutathione (GSSG) reacts with the nucleophilic cysteine (C23) of Grx1, to form a Grx1-glutathione disulfide intermediate. The next step is to S-glutathionylate the roGFP2, by reacting with one of the thiols of roGFP2. S-glutathionylated roGFP2 per se is unstable; stability is achieved by internal disulfide-bridge (C147-C204) formation (Meyer and Dick 2010). Application of oxidants $\left(\mathrm{H}_{2} \mathrm{O}_{2}\right)$ leads to conformational changes on the roGFP2 structure, due to disulfide bridge formation between $B$-strand 7 and 10. Antioxidant challenge of the sensor helps the roGFP2 protein to form thiol groups of the adjacent Cy147 and Cys 
204 (Figure 5B). The fluorescent intensity changes of the signal at 405 and $488 \mathrm{~nm}$ upon oxidation and reduction are shown below (Figure 5C).

A) GS-sG

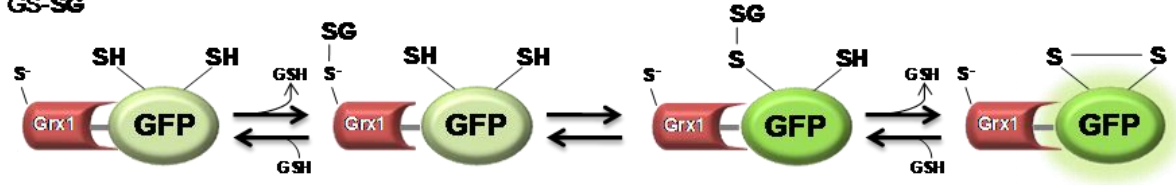

B)

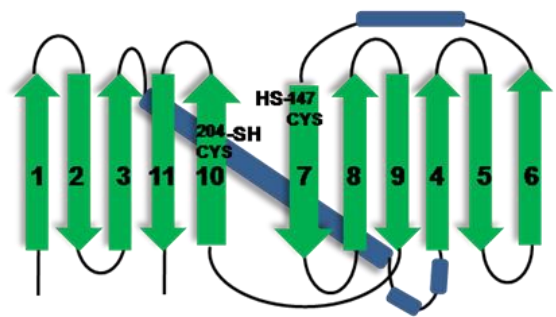

reduced roGFP2

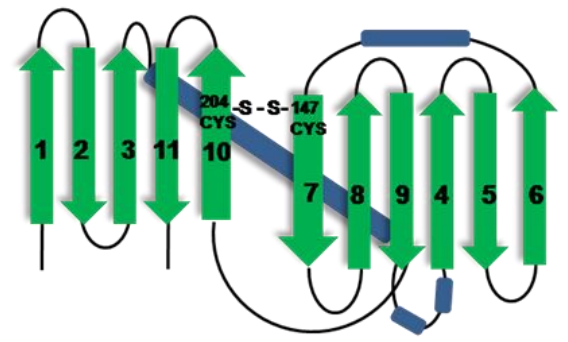

oxidized roGFP2

C)

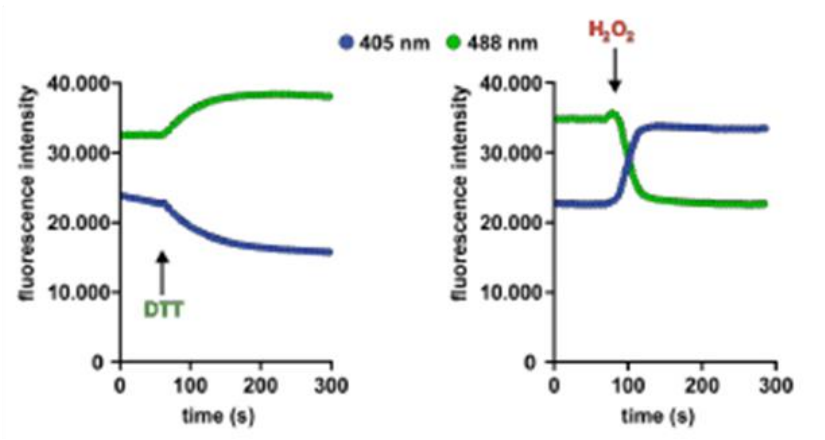

Figure 5: Schematic view of Grx1-roGFP2 sensor in oxidized and reduced conditions. (A) Reversible three step mechanism of the biosensor. (B) The B-sheet organization of reduced and oxidized roGFP2, with Cys 204 and Cys 147 on Bstrands 7 and 10. (C) Fluorescence intensities at 405 and $488 \mathrm{~nm}$ of the oxidized and reduced probe. Image adapted from (Aller et al. 2013, Meyer and Dick 2010, Swain et al. 2016).

\subsection{Drug-ROS sensing and therapeutic opportunities}

In the sections above, ROS sources and the pathophysiological role of ROS production are introduced. Another aspect to be considered is the toxicity and oxidative stress by drug-induced ROS production. 
Several classes of drugs have been investigated for cardiac toxicity and the underlying mechanism to produce ROS. For example, the anticancer drug doxorubicin (Dox) can induce mitochondrial dysfunction and lipid peroxidation due to damage of amino acids and DNA caused by ROS (Raschi et al. 2010, Sawyer et al. 2010). Azidothymidine (AZT) induces ROS and NOS production (Kline et al. 2009, Kohler et al. 2009).

Enhancers of cardiac contractility such as the calcium-sensitizer levosimendan (LEVO) and the myosin activator omecamtiv mecarbil (OME) are applied clinically in acute heart failure. Enhanced contractility typically results in enhanced ATP turnover, which may be compensated by enhanced mitochondrial ATP production as well as ROS generation. Interestingly, OME in contrast to LEVO, despite both being strong positive inotropes, seems to not increase ATP consumption. The specific mechanisms of action of LEVO include: (1) positive inotropic effect by enhanced calcium sensitivity of troponin $C$ and (2) pre- and afterload reduction as a consequence of vasodilation due to the opening of ATP-sensitive potassium channels in smooth muscle cells (Parissis et al. 2007, Pollesello et al. 2016). In addition, a cardioprotective effect has been postulated for LEVO which appears to be linked mechanistically to the activation of the mitochondrial ATP-sensitive $\mathrm{K}^{+}$-channel (Farmakis et al. 2016, Parissis et al. 2008). In non-myocytes (human neutrophils) LEVO inhibits ROS production (Hasslacher et al. 2011). Furthermore, LEVO prevents the generation of ROS by myeloperoxidase enzyme (MPO), which is released by polymorphonuclear leukocytes (PMN) in heart failure (HF) patients (Hasslacher et al. 2011). In human atrial biopsies, antioxidant effects of LEVO were reported to prevent cell death of cardiomyocytes and $\mathrm{H}_{2} \mathrm{O}_{2}$-induced contractile dysfunction (Parissis et al. 2008, Sahin et al. 2007). Collectively, these data suggest an effect of LEVO on ROS, but do not specify whether ROS alterations occur in cardiomyocytes and fibroblasts. OME, also known as CK-1827452, enhances myosin-actin cross-bridge formation without an increase in total ATP or oxygen consumption (Nagy et al. 2014, Teerlink 2009).

In contrast to OME and LEVO, Angiotensin II stimulation directly affects ROS production $\left(\mathrm{H}_{2} \mathrm{O}_{2}\right.$ and $\left.\mathrm{O}_{2}{ }^{-}\right)$by the activation of NADPH oxidases. Initially, the 
signaling starts with the activation of G-protein-coupled receptor 1 (AT1R), which activates redox dependent targets (Rac, c-Src, protein kinase C; PKC) to stimulate $\mathrm{O}_{2}^{-}$production by NOX2 in the heart. When reacting with $\mathrm{NO}$ radicals, $\mathrm{O}_{2}^{-}$produces peroxynitrite, whereas dismutation with superoxide dismutase (SOD) results in $\mathrm{H}_{2} \mathrm{O}_{2}$ production. Angll stimulation increases in cytoplasmic calcium $\left(\mathrm{Ca}^{2+}\right)$, thus activating NOX5 to produce $\mathrm{H}_{2} \mathrm{O}_{2}$. Both $\mathrm{H}_{2} \mathrm{O}_{2}$ and $\mathrm{ONOO}^{-}$then stimulate mitochondrial ROS that will activate NADPH oxidases in the cytoplasm (Figure 6) (Dikalov and Nazarewicz 2013).

Previous studies have used a number of antioxidants to reduce ROS, but the lack of specificity and disturbance of the redox homeostasis contributed to the suboptimal efficacy of the so far reported approaches (Zhang et al. 2012). Another approach was to develop specific compounds that could target and inhibit ROS production enzymes and proteins modified by ROS (Dao et al. 2015). Dao et al. further highlight the strengths of each of these strategies with promising directions such as inducing Nrf2 transcription by dimethyl fumarate (DMF) activators like BG12, an approach that has been reported to be effective in multiple sclerosis (Bomprezzi 2015, Kappos et al. 2008). In general it can be concluded that targeting the mitochondrial pathway to modulate redox states in cells appears promising in diseases with ROS dysregulation (Brown et al. 2016). Also because of its translational potential it is important to develop experimental models allowing for a clear correlation of ROS activity and function; biosensors for a quantitative assessment of redox potentials appear instrumental also for the development of cardioactive drugs or to identify potentially limiting side effects (Santos et al. 2016). Accordingly, the strategy of this study is to develop and validate a method for optical assessment of redox alterations in human heart muscle. 


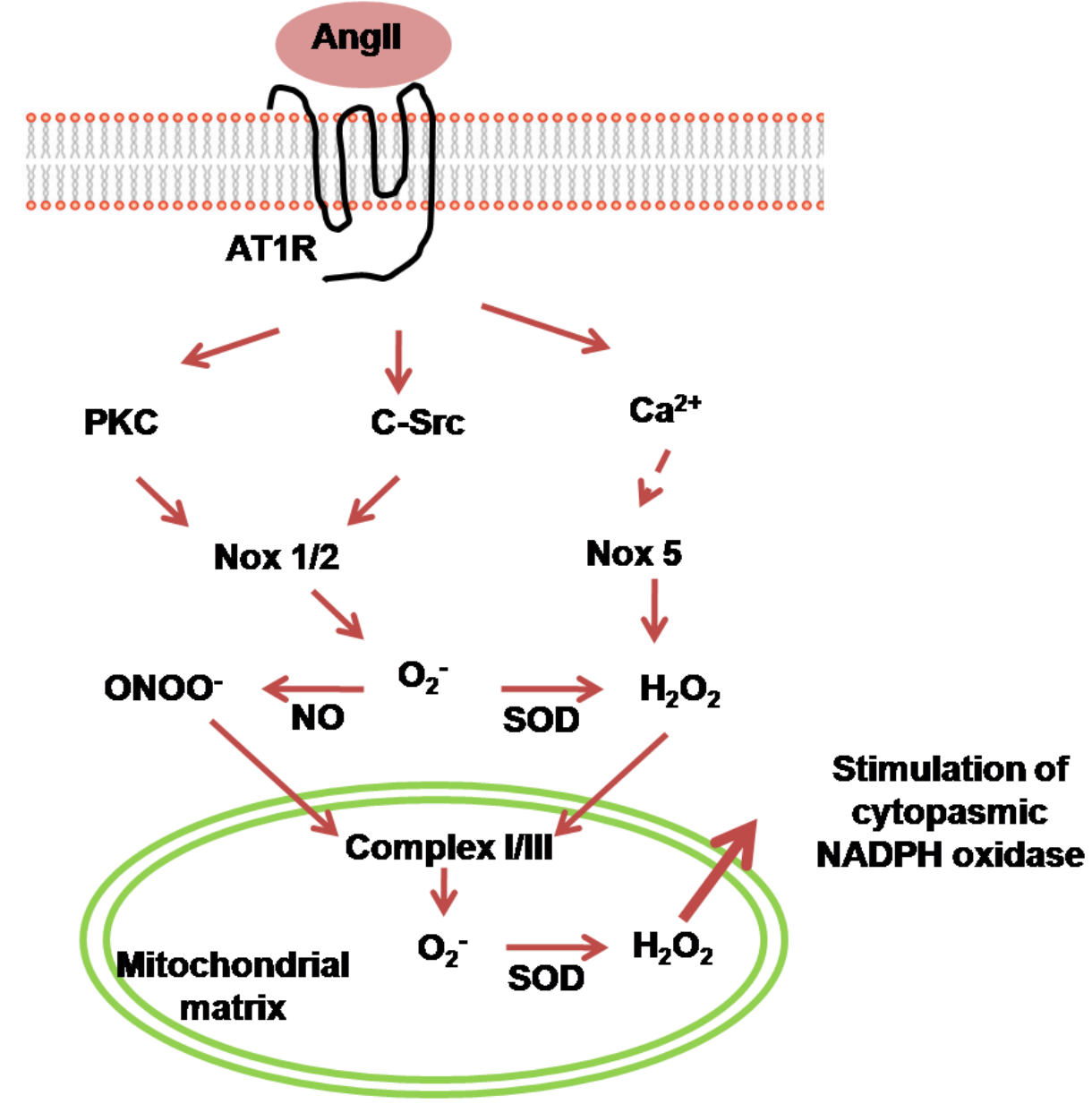

Figure 6: Redox signaling pathway via Angll stimulation. Activation of GPCR and AT1R follows the activation of several molecules that stimulate NOX specific enzymes to produce ROS. Image adapted from (Dikalov and Nazarewicz 2013).

\subsection{Engineered heart muscle as a 3D model to study redox changes}

Engineered Heart Muscles (EHM) is a promising tool for in vitro studies in cardiovascular research (Tiburcy et al. 2017). In fact, several groups have proposed different engineered heart tissue models for applications in drug development (Hirt et al. 2014). The engineering of force-generating cardiac tissues were first reported in embryonic chick (Eschenhagen et al. 1997) and neonatal rat (Zimmermann et al. 2000) models. Most recently, ring-shaped human EHM have been advanced to display several structural (for example anisotropic sarcomere assembly) and functional (for example positive force frequency relationship) properties of postnatal myocardium (Tiburcy et al. 2017). Several drugs have been tested in tissue 
engineered myocardium, including angiotensin II and anthracyclines; angiotensin II enhanced EHM stiffness without reducing contractile performance; doxorubicin demonstrated the anticipated concentration dependent cardiomyocyte toxicity (unpublished data). Whether these effects are related to ROS production has not been studied so far. The use of redox reporters would facilitate these analyses.

\subsection{Aim of the thesis}

This study aims to (Figure 7):

1) Establish stable Grx1-roGFP2 expression in human cardiomyocytes and fibroblasts to enable optogenetic recordings of GSH redox state in a cell type specific manner.

2) Determine whether GSH redox state alterations under pharmacological stimulation can be monitored in human cardiomyocytes and fibroblasts.

3) Establish cell-type specific GSH redox state imaging in a tissue (EHM) context. 


\section{Cloning of biosensor}

5

$3^{4}$

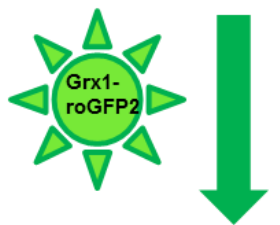

Lentiviral

transduction

$\underline{\text { Aim I }}$

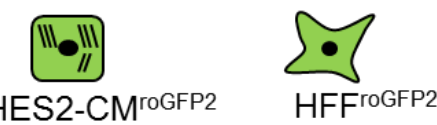

Aim II
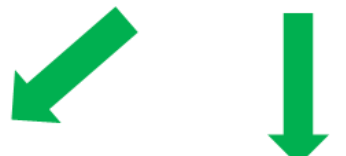

Aim III

Redox state measurements in monolayer cells

Redox state measurement in 3 dimensional EHM
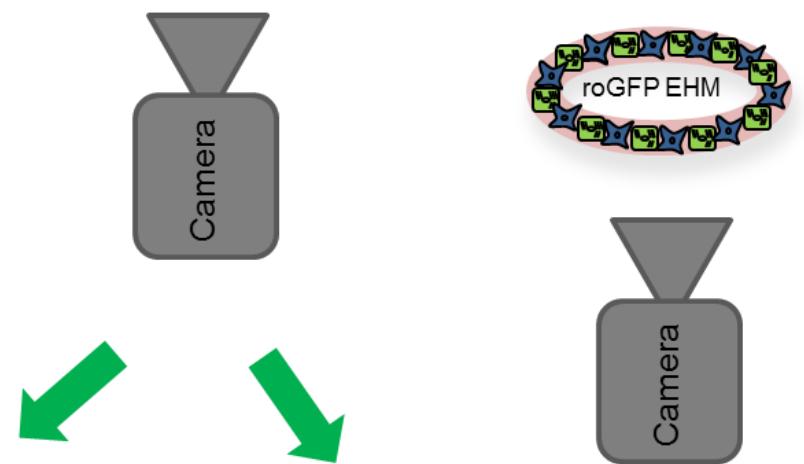

Calculations of redox potential

Visualizing drug effects on redox changes

Functional redox state measurement in EHM

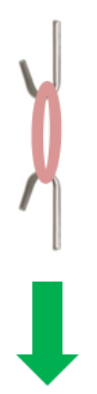

Measurement of force of contraction (FOC)

Visualizing redox changes in EHM

Figure 7: Overview of the strategy, methods, and aims used in this study. 


\section{Materials and Methods}

\subsection{Cells}

An overview of the cell lines used in this study can be found in Appendix 2. The use of human embryonic stem cells was approved according to the German Stem Cell Act by the Robert-Koch-Institute (permit \#12; reference number: 1710-79-1-4-16).

\subsubsection{TSA201}

Commercially available TSA201 cells were derived from human embryonic kidney and immortalized by transduction of the SV40 large T-antigen (Giovarelli et al. 1995). In this study, TSA cells were used to test the expression of GFP from pGIPZ-Grx1roGFP2 plasmid and as host cells of the packaging plasmids for the production of transgenic lentivirus.

\subsubsection{Cell Culture and maintenance of cell lines}

Passaging of TSA201 cells was performed at $70 \%$ confluency. The cells were rinsed with 1x PBS and incubated for 3-5 mins in TryPLE (Invitrogen, 12604) to aid cell detachment. TryPLE was inactivated by the addition of $2 x$ volume of TSA culture medium (Appendix 1) and the complete cell suspension was centrifuged at $300 \times \mathrm{g}$ for 5 mins. The cell pellet was triturated in TSA culture medium and counted using the CASY Model TT cell counter (Roche). The desired number of TSA201 cells was seeded in cell culture dishes or frozen for further usage.

\subsubsection{Freezing and thawing of TSA201 cells}

The cells were suspended $\left(3-8 \times 10^{6}\right.$ cells $\left./ \mathrm{ml}\right)$ and triturated gently in freezing medium (see Appendix 1). The cells were transferred into cryovials (1ml/vial) and placed inside a freezing box at $-80^{\circ} \mathrm{C}$ to allow for controlled freezing. The cells were finally stored at -80 or $-150{ }^{\circ} \mathrm{C}$ until further use. To thaw the cells, the cryovials were placed in $37^{\circ} \mathrm{C}$ water bath for $2-3$ mins, subsequently suspended in $9 \mathrm{ml}$ of fresh and 
warm TSA medium, and centrifuged at $300 \times \mathrm{g}$ for 5 mins to remove the DMSO. The cell pellet was further triturated in fresh TSA medium and then used for experiments.

\subsubsection{Human foreskin fibroblasts}

Human foreskin fibroblasts (HFF) were purchased from American Type Culture Collection (SCRC-1041; ATCC) and cultured in human fibroblast medium (Appendix 1). Passages 25-30 were used for all the following experiments.

\subsubsection{Culturing and passaging of HFFs}

HFFs were passaged regularly when reaching a confluency of $80-90 \%$. The cells were washed with pre-warmed (at $37^{\circ} \mathrm{C}$ ) PBS and then treated for 3-5 mins with prewarmed TryPLE (Invitrogen). To inactivate TryPLE, 2x volume of HFF medium was added to the detached cell suspension, which was triturated to produce a single cell suspension. To pellet the cells, the cell suspension was centrifuged at $300 \times \mathrm{g}$ for 5 mins. The cells were then suspended in HFF medium, counted using the CASY Model TT cell counter (Roche), and seeded on non-coated culture flasks at the required density or frozen (see Section 2.1.1.2) for storage purposes.

\subsubsection{Human embryonic stem cells}

The human embryonic stem cell line (HES2) with a genetic modification of the ROSA26 locus to express a tandem dimer red fluorescence protein (tdRFP) was kindly provided by Prof. G. Keller (Toronto) (Irion et al. 2007). These cells were cultured on $\gamma$-irradiated HFFs feeder layers and then cultured in HESC medium (see Appendix 1). HES2 cells were passaged for expansion and single cell adaptation by using EDTA digestion solution ( $0.5 \mathrm{~mol} / \mathrm{L}, \mathrm{pH} 8$, Applichem). After adaptation, these cells were differentiated into cardiomyocytes.

\subsubsection{Monolayer differentiation of HES2-CM}

Directed differentiation of HES2 was by a staged protocol (Tiburcy et al. 2017). Initially, HES cells were plated at a density of $5-10 \times 10^{5}$ cells $/ \mathrm{cm}^{2}$ on feeder free 
Matrigel $^{\mathrm{TM}}$ (growth factor reduced; 1:60 diluted in 1x PBS; BD Biosciences)-coated flasks and cultured in HES cell-conditioned medium (Appendix 1) for 24 hours. HES cells were then rinsed with RPMI medium (Appendix 1) and kept in mesoderminduction medium (Appendix 1) for 3 days. HES cells were then rinsed with RPMI medium and cultured in mesoderm specification medium (Appendix 1) for 10 days. Highly purified cardiomyocytes were subsequently obtained with metabolic selection (Tohyama et al. 2013) in HES selection medium $\left(0.28 \mathrm{ml} / \mathrm{cm}^{2}\right.$; Appendix 1).

\subsubsection{Single cell dissociation of HES2-CM}

The enriched cardiomyocytes obtained from the differentiation procedure (section 2.1.3.1) were washed 2 times with $1 \mathrm{x}$ PBS and treated with Accutase digestion solution (Appendix 1) for 10 mins at RT, until the cells were detached from the flasks into single cells. The digestion solution was inactivated with 2-3 times the volume of serum-free $\mathrm{CM}$ medium and $5 \mu \mathrm{mol} / \mathrm{L}$ of Rock inhibitor (Appendix 1 ) was added into the cell solution. The harvested cells were centrifuged at $200 \times \mathrm{g}$ for 10 mins and further triturated in fresh serum-free medium. The cells were either re-plated onto Matrigel $^{\mathrm{TM}}$-coated flasks or used for further experiments. The purity of cardiomyocytes was determined by antibody labelling of sarcomeric $\alpha$-actinin and assessed by Flow cytometry analysis software (Section 2.1.4; see Table 8 for antibody dilution and immunostaining protocol).

\subsubsection{Freezing and thawing of HES2-CM}

Cells were re-suspended $\left(3-8 \times 10^{6}\right.$ cells $\left./ \mathrm{ml}\right)$ and triturated gently in freezing medium (Appendix 1). To thaw the frozen cardiomyocytes, the cryovial was warmed up 2-3 mins in a $37^{\circ} \mathrm{C}$ water bath and gently triturated via a $2 \mathrm{ml}$ pipette in $9 \mathrm{ml}$ fresh $\mathrm{CM}$ medium. The cell suspension was centrifuged at $200 \mathrm{xg}$ for 10 mins to remove DMSO. The cell pellet was re-suspended in fresh serum-free $\mathrm{CM}$ medium and plated onto Matrigel ${ }^{\mathrm{TM}}$-coated flasks to recover for at least 5 days. 


\subsubsection{Flow cytometry}

Flow cytometry analysis was performed on both live and cells fixed in $70 \%$ ice-cold ethanol or fixed in 4\% PFA for 15 mins at RT.

\subsubsection{Live cell analysis}

Live analysis was done to analyse the transduction efficiency in live cardiomyocytes and fibroblasts. In live cell analysis, the transduced and non-transduced cells were washed once with $1 \times$ PBS and then centrifuged at $300 \times \mathrm{g}$ for 5 mins. Cell pellets were washed with blocking buffer containing 5\% FBS (Appendix) and centrifuged at $300 \times \mathrm{g}$ for 5 mins. Meanwhile, Sytox solution was prepared (1:1,000 dilution) in blocking buffer with 5\% FBS. The cells were then incubated in Sytox solution for 15 mins at RT. After the incubation time, the cells were washed with 1x PBS and centrifuged at $300 \mathrm{xg}$ for 5 mins. After 2 repeats of washing and pelleting, the cells were strained through a $70 \mu \mathrm{m}$ cell strainer to remove cell clumps and subjected to flow cytometry analysis (LSR II Cytometer, BD Bioscience).

\subsubsection{Fixed cell suspensions analysis}

Cell suspensions previously fixed in ice-cold $70 \%$ ethanol or $4 \%$ Roti $^{\circledR}$ Histofix, were strained though a $70 \mu \mathrm{m}$ cell strainer and subjected to blocking buffer solution (Appendix 1) to permeabilize the cells for 10 mins at RT. The cells were incubated with specific primary antibodies for 45 mins at $4{ }^{\circ} \mathrm{C}$ (Table 8). At this step, to determine the purity of the differentiated cardiomyocyte population, CM-specific sarcomeric a-actinin antibody incubation was performed (Sigma-Aldrich, A7811, 1:4,000; Table 8). Cell populations used as a negative control were incubated with a primary antibody against Immunoglobulin $G$ ( $(g G)$ isotype control (R\&D systems). After the incubation, the cells were washed (2x) with blocking buffer solution and then incubated with secondary antibody and the nuclei staining dye Hoechst (Invitrogen, $\mathrm{H} 2570 ; 1: 1,000)$ in the dark for $1 \mathrm{hr}$ at $4{ }^{\circ} \mathrm{C}$. For $\mathrm{CM}$, goat anti-mouse Alexa 488 (Invitrogen, A-110001; 1:1,000) was used as a secondary antibody. The cells were washed again $(2 x)$ in blocking buffer solution and re-suspended in PBS. The analysis 
was performed using a LSR II Cytometer (BD Bioscience) and FACSDiva Software 6.0 (BD Bioscience).

\subsection{Cloning of pGIPZ- Grx1-roGFP2}

The original plasmid pLPCX-Grx1-roGFP2 containing the biosensor coding sequence "Grx1-roGFP2" was a kind gift from Prof. Tobias Dick. The Grx1-roGFP2 coding sequence was excised and inserted into the pGIPZ lentiviral backbone (Open Biosystems). The cloning strategy is briefly described in Figure 8.

(A) PCR amplification of "CMV-Grx1-roGFP2" by integrating Xbal and Notlrestriction enzyme cutting sites. To amplify the sequence of interest "Grx1-roGFP2", forward and reverse primers with flanking restriction enzyme cutting sites on 5' and 3' ends were designed (Table 1). The amplified PCR product (5'-Xbal restriction site - CMV-Grx1-roGPF2 - Notl restriction site-3') was integrated into the multiple cloning site (MCS) of a TOPO vector. 

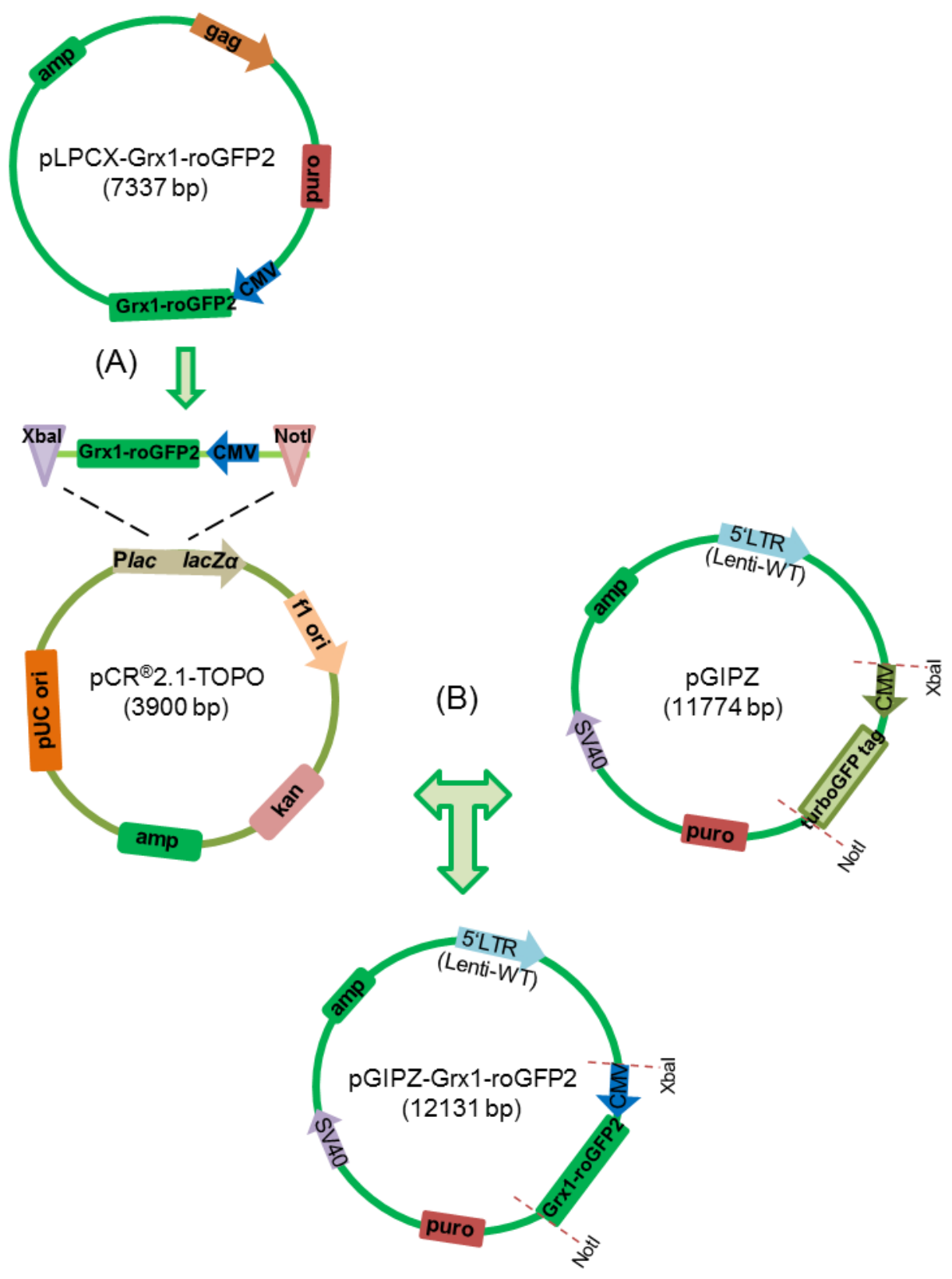

Figure 8: Schematic overview of the cloning strategy. (A) The CMV-Grx1roGFP2 sequence of the original plasmid provided by Prof. Dick was PCR amplified and decorated with flanking Xbal and Notl restriction sites. The PCR product was inserted into a TOPO vector. (B) The CMV-turboGFP sequence in PGIPZ was replaced with CMV-Grx1-roGFP2 from TOPO vector.

(B) Replacement of the CMV-turboGFPtag from the pGIPZ backbone by CMV-Grx1roGFP2 sequence with Xbal and Notl restriction cutting sites at 5 ' and 3 ' ends respectively. Restriction enzymes Xbal and Notl were used to remove CMV- 
turboGFP sequence from the pGIPZ backbone. The CMV-Grx1-roGFP2 sequence was inserted via corresponding $\mathrm{Xbal}$ and Notl restriction sites yielding the plasmid pGIPZ-Grx1-roGFP2.

\begin{tabular}{|l|l|l|}
\hline \multicolumn{2}{|l|}{ Forward primers (sequence $\mathbf{5}^{\prime}$-' $\mathbf{3}^{\prime}$ ) } \\
\hline \multicolumn{2}{|c|}{ XBalkozakCMV } & Base pairs (bp) \\
\hline F1 & $\begin{array}{l}\text { Gctctagagcagatagtaatcaattacgggg } \\
\text { tc }\end{array}$ & 33 \\
\hline F2 & $\begin{array}{l}\text { Gctctagagcagggccaccatagtaatcaat } \\
\text { tacgg }\end{array}$ & 36 \\
\hline F3 & gctctagagcagatagtaatcaattacgg & 29 \\
\hline
\end{tabular}

Reverse primer (sequence 5'-3')

\begin{tabular}{|l|l|l|}
\hline \multicolumn{2}{|l|}{ Not IGrx1-roGP2 } & Base pairs (bp) \\
\hline$R$ & $\begin{array}{l}\text { Ataagaatcgccggcgtaaactatttacttgta } \\
\text { cagctcgtc }\end{array}$ & 42 \\
\hline
\end{tabular}

Table 1: Forward and reverse primers used for PCR amplification of CMV-Grx1roGFP2. Primers $\mathrm{F} 1$ and $\mathrm{R}$ were chosen for PCR amplification.

Each of the cloning steps includes technologies such as Polymerase Chain Reaction (PCR), RE digestion, agarose gel electrophoresis and extraction, ligation, transformation, plasmid DNA preparation, and DNA sequencing. The details of each of the techniques are explained in the following sections.

\subsubsection{Polymerase Chain Reaction (PCR)}

PCR amplifications of the biosensor coding sequence were done by using a $T$ gradient PCR thermocycler (Biometra). The forward primers (F1-3) shown in Table 1 were all used in combination with the reverse primer R. Parameters and the amounts of each of the components are listed in Table 2 below. 


\begin{tabular}{|l|c|c|c|c|}
\hline & A & B & C $^{*}$ & D \\
\hline Primer combination & F1 \& R & F2 \& R & F2 \& R & F3 \& R \\
\hline $\mathrm{dH}_{2} \mathrm{O}(\mu \mathrm{l})$ & 37.7 & 37.7 & 37.7 & 37.7 \\
\hline Ex Taq10x Buffer $(\mu \mathrm{l})$ & 5 & 5 & 5 & 5 \\
\hline dNTPmix $(2.5 \mathrm{mmol} ; \mu \mathrm{l})$ & 4 & 4 & 4 & 4 \\
\hline Template $(\sim 200 \mathrm{ng} ; \mu \mathrm{l})$ & 1 & 1 & 1 & 1 \\
\hline Primer F $(10 \mathrm{mmol} ; \mu \mathrm{l})$ & 1 & 1 & 1 & 1 \\
\hline Primer $\mathrm{R}(10 \mathrm{mmol} ; \mu \mathrm{l})$ & 1 & 1 & 1 & 1 \\
\hline ExTaq Enzyme $(5 \mathrm{U} / \mu \mathrm{l} ; \mu \mathrm{l})$ & 0.3 & 0.3 & 0.3 & 0.3 \\
\hline TOTAL $(\mu \mathrm{l})$ & 50 & 50 & 50 & 50 \\
\hline
\end{tabular}

Table 2: Components and parameters needed for PCR. ${ }^{*}$ The difference to $B$ is the annealing temperature $\left(55^{\circ} \mathrm{C}\right)$.

The time and temperature protocol for each of the primer combinations is shown in Table 3 below.

\begin{tabular}{|c|c|c|c|c|c|c|}
\hline Program & \multicolumn{4}{|c|}{ Temperature $\left({ }^{\circ} \mathrm{C}\right)$} & Time (sec) & Cycles \\
\hline Initial denaturation & \multicolumn{4}{|c|}{98} & 30 & 1 \\
\hline Denaturation & \multicolumn{4}{|c|}{98} & 10 & \multirow{4}{*}{30} \\
\hline \multirow{2}{*}{ Annealing } & A & B & C & D & \multirow{2}{*}{30} & \\
\hline & 66 & 66 & 55 & 61 & & \\
\hline Elongation & \multicolumn{4}{|c|}{72} & 30 & \\
\hline Final elongation & \multicolumn{4}{|c|}{72} & 120 & 1 \\
\hline End of program & \multicolumn{4}{|c|}{4} & $\infty$ & 1 \\
\hline
\end{tabular}

Table 3: PCR protocol. Except of annealing temperature, all the other steps were identical for each primer combination.

All PCR products were finally mixed with 6x DNA loading buffer (New England Biolabs, NEB) and separated by $1 \%$ agarose gel electrophoresis (Appendix 1 ). 


\subsubsection{Agarose gel electrophoresis}

To prepare a $1 \%$ agarose gel, $3 \mathrm{~g}$ of agarose was dissolved into $300 \mathrm{ml}$ of $1 \mathrm{x}$ TAE running buffer (Appendix 1). The cooling of the gel solution was followed by the addition of $0.2 \mu \mathrm{g} / \mathrm{ml}$ of ethidium bromide (EtBr; Sigma Aldrich, \#1239-45-8). The solution was poured into a gel tray and left to polymerise for 30 mins. Next, PCR amplified samples were loaded together with a $1 \mathrm{~kb}$ DNA ladder (Plus; Fermentas). Electrophoresis was at $100 \mathrm{~V}$ for 45 mins in $1 \times$ TAE running buffer (Appendix 1). Visualization of the DNA was under UV in the Gel Doc XR (BioRad) with subsequent analysis using the Quantity One Software (BioRad).

\subsubsection{Plasmid DNA extraction from agarose gels}

Extraction of DNA bands from agarose gels was performed as per manufacturer's protocol (QIAquick gel extraction kit, QIAGEN). First, the desired DNA fragments were excised with a clean scalpel and put into $1.5 \mathrm{ml}$ polypropylene (PP) tubes (Eppendorf). Each of the gel sections containing the DNA bands was weighed and $300 \mu \mathrm{l}$ of Buffer QG was added per $100 \mathrm{mg}$ of gel. Subsequently, the samples were incubated at $50{ }^{\circ} \mathrm{C}$ for 10 mins with vortex intervals every 2-3 minutes. Complete dissolving of the gel was accompanied with the addition of $100 \mu \mathrm{l}$ of isopropanol per $100 \mathrm{mg}$ of gel. The solution was placed into QIAquick $2 \mathrm{ml}$ spin columns and centrifuged at $13,000 \times \mathrm{g}$ for $1 \mathrm{~min}$. The flow-through was discarded and the QIAquick columns were washed with $0.75 \mathrm{ml}$ Buffer PE. The washing step was followed by spinning at $13,000 \times \mathrm{g}$ for $1 \mathrm{~min}$ and the QIAquick columns were placed into clean $1.5 \mathrm{ml}$ PP tubes. $30 \mu \mathrm{l}$ Buffer EB or pre-warmed $\left(50{ }^{\circ} \mathrm{C}\right) \mathrm{H}_{2} \mathrm{O}$ was added into the columns and left to stand for $1 \mathrm{~min}$. The columns were centrifuged again at $13,000 \times \mathrm{g}$ for $1 \mathrm{~min}$ and the concentration of the eluted DNA was measured by using a spectrophotometer (Nanodrop, ND-1000, Thermo Scientific).

\subsubsection{Ligation}

The DNA fragments eluted from the PCR of each primer combination (Table 4) were subjected to ligation reaction with either a TOPO vector (Figure $8 \mathrm{~A}$ ) or the $\mathrm{pGIPZ}$ 
backbone (Figure 8B). For an optimal ligation reaction, the size and concentration of the vector and the insert was calculated with the formula below:

$$
\operatorname{Insert}(n g)=\operatorname{Vector}(n g) \times \frac{\operatorname{Insert}(b p)}{\operatorname{Vector}(b p)} \times \text { ratio }
$$

Subcloning into TOPO vector was performed according to the manufacture's protocol. Ligation was performed by incubating the insert and the vector with T4 ligase (NEB) at $16{ }^{\circ} \mathrm{C}$ overnight. The components of the ligation reactions for Figure $8 \mathrm{~A}$ and $\mathrm{B}$ are shown in Table 4.

\begin{tabular}{|l|c|}
\hline PCR A (CMV-Grx1-roGFP2) ligation to TOPO vector (Figure 8A) \\
\hline Insert (PCR product; $100 \mathrm{ng} / \mu \mathrm{l} ; \mu \mathrm{l})$ & 1 \\
\hline Salt solution $(\mu \mathrm{l})$ & 1 \\
\hline TOPO vector $(10 \mathrm{ng} / \mu \mathrm{l} ; \mu \mathrm{l})$ & 3 \\
\hline $\mathrm{dH}_{2} \mathrm{O}(\mu \mathrm{l})$ & 6 \\
\hline Total $(\mu \mathrm{l})$ & \\
\hline
\end{tabular}

PCR A (CMV-Grx1-roGFP2) ligation to pGIPZ (Figure 8B)

\begin{tabular}{|l|c|}
\hline Vector $(\mathrm{pGIPZ} ; 50 \mathrm{ng} / \mu \mathrm{l} ; \mu \mathrm{l})$ & 1.5 \\
\hline Insert (PCR A; CMV-Grx1-roGFP2; $\mu \mathrm{l})$ & 0.5 \\
\hline $10 \times \mathrm{T} 4$ ligase Buffer $(\mu \mathrm{l})$ & 2 \\
\hline T4 ligase $(\mu \mathrm{l})$ & 15 \\
\hline $\mathrm{dH}_{2} \mathrm{O}(\mu \mathrm{l})$ & 20 \\
\hline Total $(\mu \mathrm{l})$ & \\
\hline
\end{tabular}

Table 4: Ligation of insert and vector (step A-B as indicated in Figure 8).

\subsubsection{Transformation}

$5 \mu \mathrm{l}$ of the ligation mixture was added into $100 \mu \mathrm{l}$ of TOP10 competent cells and incubated on ice for 30 mins. This step was followed by heat shock of the cells for 60 sec at $42{ }^{\circ} \mathrm{C}$ with subsequent placement on ice for 2 mins. $450 \mu$ of SOC medium (Invitrogen) was added into the mixture followed by incubation while shaking at $1 \times \mathrm{g}$ for $1 \mathrm{hr}$ at $37^{\circ} \mathrm{C}$. $50-100 \mu \mathrm{l}$ of the transformation reaction solution was plated onto 
LB-agarose plates with $100 \mu \mathrm{g} / \mathrm{ml}$ ampicillin (Appendix 1) and allowed to air dry. The inverted plates were then incubated overnight at $37^{\circ} \mathrm{C}$.

For TOPO vector subcloning mixtures, except for the plating on LB-agarose plates, all previously mentioned steps were the same. 30 minutes before plating the ligation mixture, $40 \mu \mathrm{l}$ of $X$ gal (5-bromo-4-chloro-3-indolyl- $\beta$-D-galactopyranoside) and $40 \mu \mathrm{l}$ of IPTG (Isopropyl $\beta$-D-1-thiogalactopyranoside) were plated onto the LB-agarose plates with ampicillin resistance. Using the blue-white colony screening strategy, detection of positive colonies (white coloured colonies) from negative colonies (blue colonies) can be performed. After plating the ligation mixture, the plates were incubated upside down at $37^{\circ} \mathrm{C}$ in a dark environment.

\subsubsection{Miniplasmid DNA preparation}

The procedure for small scale plasmid DNA amplification was performed according to manufacturer's protocol with NucleoSpin ${ }^{\circledR}$ Plasmid columns (Macherey-Nagel, \#740499.250). First, $50 \mathrm{ml} \mathrm{PP}$ tubes (Falcon, BD) were filled with $4 \mathrm{ml}$ LB-medium (Lysogeny broth; Appendix 1) and $100 \mu \mathrm{g} / \mathrm{ml}$ ampicillin. The colonies were picked from the agar plates and transferred into the LB-medium and incubated overnight (8$12 \mathrm{hrs}$ ) at $37^{\circ} \mathrm{C}$ and shaking at $220 \mathrm{rpm}$. The next day, the cultures were centrifuged at $11,500 \times \mathrm{g}$ for $30 \mathrm{sec}$. After spinning, the pellet of bacteria was re-suspended into $250 \mu \mathrm{l}$ of Buffer A1 (Macherey-Nagel) and mixed with $250 \mu \mathrm{l}$ of lysis Buffer A2 (Macherey-Nagel). The mixture was inverted 6-8 times and then incubated at RT for 5 mins. After lysis, $300 \mu \mathrm{l}$ of neutralisation Buffer A3 (Macherey-Nagel) was added into the lysed bacteria and the mixture was again inverted 6-8 times. In the next step, the mixture was centrifuged at $11,500 \times \mathrm{g}$ for 5-10 mins. The supernatant obtained from the last centrifugation was transferred into the Nucleospin collection tubes. The supernatants were centrifuged at the same speed as before for 1 min. The flowthrough was discarded from the collection tubes. The silica membranes of the Nucleospin columns were washed first with $500 \mu \mathrm{l}$ of Buffer AW (Macherey-Nagel) and later with $600 \mu \mathrm{l}$ of Buffer A4 (Macherey-Nagel). Both steps were followed by centrifugation at $11,500 \times \mathrm{g}$ for $1 \mathrm{~min}$. The drying process of the silica membrane was done by another centrifugation for 2 mins. At the end, $30 \mu \mathrm{l}$ of pre-warmed (at $50^{\circ} \mathrm{C}$ ) $\mathrm{H}_{2} \mathrm{O}$ was added to the center of the silica membrane and left to stand at $\mathrm{RT}$ for $1 \mathrm{~min}$. 
After spinning at $11,500 \times \mathrm{g}$ for $1 \mathrm{~min}$, the eluted DNA was measured by a spectrophotometer (Nanodrop, ND-1000, Thermo Scientific) and subjected to RE digestion (2.2.7) or gel electrophoresis (section 2.2.2).

\subsubsection{Restriction enzyme digestion}

This technique was used to linearize the DNA plasmid to prepare for the ligation (CMV-Grx1-roGFP2 to pGIPZ, Figure 8B) and it can also be used for confirming the right clone/plasmid after a ligation step (subcloning of Grx1-roGFP2 into TOPO vector, Figure $8 \mathrm{~A}$ ). The reaction components and quantities required for the restriction enzyme digestion are shown in Table 5.

\begin{tabular}{|l|c|c|}
\hline \multicolumn{1}{|c|}{ Reagmids } & $\begin{array}{r}\text { Reaction I }(\mu \mathrm{l}) \\
\text { TOPO- CMV-Grx1-roGFP2 } \\
(2,000 \mathrm{ng})\end{array}$ & $\begin{array}{c}\text { Reaction II }(\mu \mathrm{l}) \\
\text { pGIPZ } \\
(4,000 \mathrm{ng})\end{array}$ \\
\cline { 2 - 3 } & 3.1 & 2.2 \\
\hline Xbal & 1 & 1 \\
\hline Notl & 1 & 1 \\
\hline Fast digest Buffer (green) & 2 & 13.8 \\
\hline $\mathrm{H}_{2} \mathrm{O}$ & 12.9 & 20 \\
\hline Total amount & 20 & \\
\hline
\end{tabular}

Table 5: Restriction enzyme digestion (figure 8B).

DNA plasmids were linearized upon incubation with fast digest restriction enzymes at $37^{\circ} \mathrm{C}$ for $1 \mathrm{hr}$. Following the incubation, the linearized DNA plasmids were subjected to gel electrophoresis and the fragments were eluted as described in Section 2.2.3. The next step was integration of "CMV-Grx1-roGFP2" into pGIPZ backbone via ligation (Section 2.2.4). 


\subsubsection{Maxiplasmid DNA preparation}

After confirming the eluted plasmid DNAs, the next step was to purify high yields of the plasmid DNA. This step was performed according to the NucleoBond ${ }^{\circledR}$ Xtra kit (Macherey-Nagel). At first, a starter culture $(4 \mathrm{ml})$ was prepared as described above (Section 2.2.6) with $100 \mu \mathrm{g} / \mathrm{ml}$ ampicillin. Incubation in a shaker (220 rpm) at $37^{\circ} \mathrm{C}$ was for $\sim 8 \mathrm{hrs}$. Once the bacteria reached exponential proliferation (log phase), $1 \mathrm{ml}$ from the starter culture was added into $250 \mathrm{ml}$ of LB-medium (containing $100 \mu \mathrm{g} / \mathrm{ml}$ ampicillin) in an Erlenmeyer flask and incubated in a shaker (220 rpm) for 12-16 hrs at $37^{\circ} \mathrm{C}$. The bacterial solution was centrifuged in an ultracentrifuge at $6,000 \times \mathrm{g}$ for 15 mins at $4{ }^{\circ} \mathrm{C}$. The supernatant was discarded and the pellet was resuspended in $12 \mathrm{ml}$ of Buffer RES + RNase A (Macherey-Nagel). This step was followed with the addition of $12 \mathrm{ml}$ of Buffer LYS, which helped in lysing the bacteria. The lysate was gently inverted 5 times and it was incubated at RT for 5 mins. Next, the NucleoBond ${ }^{\circledR}$ Xtra Column and the filter were prepared and equilibrated by $25 \mathrm{ml}$ of the Buffer EQU (Macherey-Nagel). After 5 mins of incubation, the bacterial lysate was neutralized with12 $\mathrm{ml}$ Buffer NEU (Macherey-Nagel) and gently inverted for a few times. The lysate was then poured through the equilibrated NucleoBond ${ }^{\circledR}$ Xtra Column filter and left to run until the liquid lysate was all filtered. Both the NucleoBond ${ }^{\circledR}$ Xtra Column and the filter were washed with $12 \mathrm{ml}$ of Buffer EQU and then the filter was discarded. This was followed by washing of the NucleoBond ${ }^{\circledR}$ Xtra Column with $25 \mathrm{ml}$ of Buffer WASH (Macherey-Nagel). DNA elution was done by adding $15 \mathrm{ml}$ of Buffer ELU (Macherey-Nagel) and it was collected in a $50 \mathrm{ml}$ falcon tube (BD). To precipitate the eluted DNA, $10.5 \mathrm{ml}$ of RT isopropanol was added and the elution was first mixed thoroughly by vortexing, followed by centrifugation at $8,000 \times \mathrm{g}$ for 30 mins at $4{ }^{\circ} \mathrm{C}$. The supernatant was discarded and DNA pellet was washed with $70 \%$ ethanol at RT. The plasmid solution was centrifuged at $8,000 \times \mathrm{g}$ for 15 mins at RT. The resulting DNA pellets were left to dry ( $\sim 30 \mathrm{mins})$ and then reconstituted in $100 \mu \mathrm{l}$ Elution Buffer (Macherey-Nagel). The yield of the DNA was determined by a spectrophotometer (Nanodrop, ND-1000, Thermo Scientific). 


\subsubsection{DNA sequencing}

To ensure that the cloning process was successful and that the cloned constructs were correct, plasmid DNA was prepared for sequencing (Table 6) and then sent to sequencing by SeqLab (Göttingen). The primers used for sequencing were: forward primer 5'-gctctagagcagatagtaatcaattacgg-3' and reverse primer 5'ataagaatcgccggcgtaaactatttacttgtacagctcgtc-3'.

\begin{tabular}{|l|c|c|c|c|}
\hline Clones (\#) & $\begin{array}{c}\text { Plasmid DNA } \\
(\mathbf{1}, \mathbf{2 0 0} \mathbf{n g} / \boldsymbol{\mu l} ; \boldsymbol{\mu l})\end{array}$ & Primers $(\boldsymbol{\mu l})$ & $\mathbf{d H}_{\mathbf{2}} \mathbf{O}(\boldsymbol{\mu l})$ & Total $(\boldsymbol{\mu l})$ \\
\hline 2 & 1.4 & 3 & 10.6 & 15 \\
\hline 3 & 1.2 & 3 & 10.8 & 15 \\
\hline 5 & 1.2 & 3 & 10.8 & 15 \\
\hline 7 & 1.2 & 3 & 10.8 & 15 \\
\hline 8 & 1.5 & 3 & 10.5 & 15 \\
\hline 9 & 1.2 & 3 & 10.8 & 15 \\
\hline 15 & 1.1 & 3 & 10.9 & 15 \\
\hline 21 & 1.3 & 3 & 10.7 & 15 \\
\hline
\end{tabular}

Table 6: DNA sequencing reaction mixture.

\subsection{Transduction of Grx1-roGFP2 in HFFs and HES2-CM}

Stable transduction of Grx1-roGFP2 was achieved by lentiviral vector using standard protocols.

\subsubsection{Lentivirus production}

TSA201 were seeded in $10 \mathrm{~cm}$ cell culture dishes (Nunc) in low serum medium $(0.5 \%$ FCS, 1\% P/S; see Appendix). Once 70\% confluency was reached, TSA201 cells were carefully washed once with PBS. Subsequently, $7 \mathrm{ml}$ of the low serum medium was added. Transfection of pGIPZ-Grx1-roGFP2 together with psPAX2 (Addgene plasmid \#12260) and pMD2.G (Addgene plasmid \#12259) for lentiviral packaging 
and VSV-G envelope expression (psPAX2 and pMD2.G were a kind gift by Prof. D. Trono), was done by using Polyfect reagent (Qiagen) according to manufacturer's protocol (Table 7).

\begin{tabular}{|l|c|}
\hline Components for transfection & Quantity \\
\hline pMD2.G & $2 \mu \mathrm{g}$ \\
\hline psPAX.2 & $3 \mu \mathrm{g}$ \\
\hline GOI (pGIPZ-Grx1-roGFP2) & $3 \mu \mathrm{g}$ \\
\hline DMEM (w/o serum, w/o pen/strep) & up to $300 \mu \mathrm{l}$ \\
\hline Polyfect & $80 \mu \mathrm{l}$ \\
\hline Low serum medium $(0.5 \%$ FCS, $1 \% \mathrm{P} / \mathrm{S})$ & up to $1 \mathrm{ml}$ \\
\hline
\end{tabular}

Table 7: Transfection reaction mixture for lentivirus production.

The mixture was mixed by vortexing and then incubated for 10 mins at RT. Thereafter, low serum medium up to $1 \mathrm{ml}$ was added and the complete mixture was layered onto TSA201 cells in a dropwise manner. After $72 \mathrm{hrs}$, the virus suspension was collected from the culture dishes into a $50 \mathrm{ml}$ PP (polypropylene) tube (BD, Bioscience) and filtered through $0.45 \mu \mathrm{m}$ filter (Millex ${ }^{\circledR}$ Syringe filter units, $30 \mathrm{~mm}$; Merck Millipore) to remove cell debris. The virus suspension could be directly used to transduce HFFs and HES2-CMs. Alternatively virus was purified, concentrated, and frozen at $-80^{\circ} \mathrm{C}$ until further use.

\subsubsection{Lentivirus purification}

Purification of lentivirus was done by using a commercial Vivapure ${ }^{\circledR}$ Lentiselect 40 kit (Sartorius; \#VS-LVPQ040). The virus suspension obtained after $72 \mathrm{hrs}$ of transfection (section 2.3.1) was passed slowly through a membrane absorber and collected as demonstrated in Figure 9. 


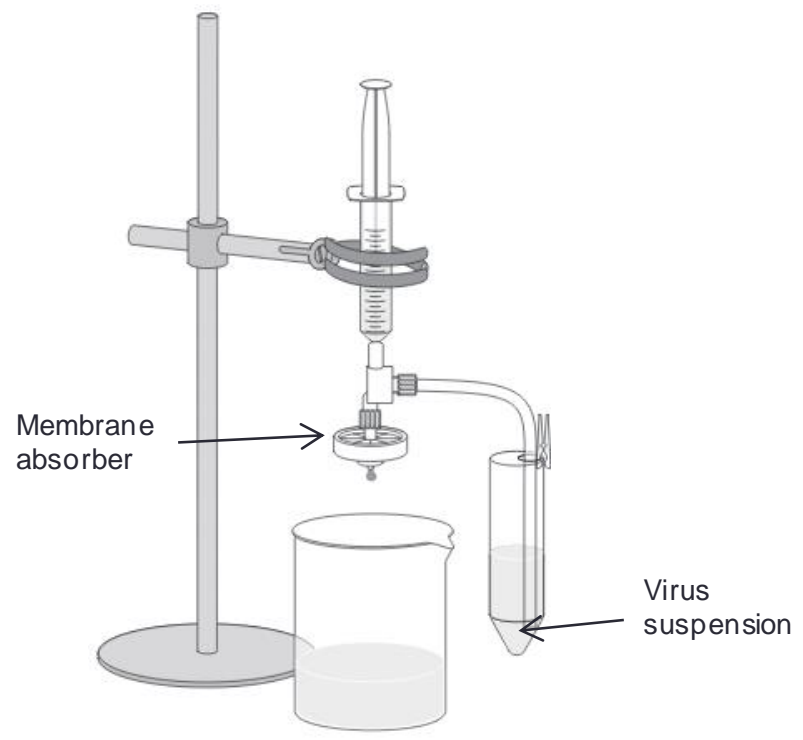

Figure 9: The assembled unit for lentivirus purification. The syringe is slowly filled with virus from the virus suspension and then passes carefully through the membrane absorber. Image adapted from (Vivapure ${ }^{\circledR}$ Lentiselect, Sartorius).

After the virus suspension was filtered, the membrane absorber was washed and then eluted with $4 \mathrm{ml}$ elution buffer according to the manufacturer's instructions. The eluted viral solution was spun at $3,000 \times \mathrm{g}$ for 12 mins. The pellet was washed $1 \mathrm{x}$ with PBS and again spun at 3,000 $\mathrm{xg}$ for 12 mins. The titer of the lentiviral particles was determined to be $>5 \times 10^{6} \mathrm{IFU} / \mathrm{ml}$ by using LentiXTM Go StixTM (Clontech). The concentrated virus was aliquoted and stored at $-80^{\circ} \mathrm{C}$.

\subsubsection{Lentivirus transduction of HFFs and HES2-CMs}

HFFs and HES2-CM at 70\% confluence were transduced with lentivirus particles. On the day of the transduction, fresh medium (for HFF human fibroblast medium; for HES2-CM-serum free CM medium; see Appendix 1) together with polybrene (0.8 $\mathrm{mg} / \mathrm{mL}$; see Appendix 1) was added onto the cells. On HFFs, fibroblast growth factor (FGF; 10ng/ml; Appendix1) was also added to the medium. Frozen aliquots of Grx1roGFP2 - lentivirus (100 $\mu$ l/each) were thawed on ice and added to the medium inside the flasks. The cells were incubated with the virus for $72 \mathrm{hrs}$ at $5 \% \mathrm{CO}_{2}$ and 37 ${ }^{\circ} \mathrm{C}$. After the transduction process, the medium of the HFFs was changed to fresh 
human fibroblast medium containing $1 \mu \mathrm{g} / \mathrm{ml}$ of puromycin (Invitrogen, \#A11138-03) to select for successfully transduced cells. We initiated the selection of HES2-CM cultures with $0.1 \mu \mathrm{g} / \mathrm{ml}$ of puromycin, however after $48 \mathrm{hrs}$ the cells were observed to be of poor quality and puromycin was subsequently withdrawn.

\subsection{Redox measurements of transgenic HFFs and HES2-CMs}

The transduced HFFs and HES2-CMs (section 2.4.4) were seeded in 24-well imaging plates (Zell Kontakt, \#3231-20). For HES2-CMs, the imaging plate was first coated with Matrigel ${ }^{\mathrm{TM}}$ (1:120 in PBS) and incubated at $37^{\circ} \mathrm{C}$ for 30 mins. The transgenic CMs were seeded as above (section 2.1.3.2). For HFFs, the seeding process in the imaging plate was performed as before (section 2.1.2.1).

The redox measurements on the transgenic cells were done by using an inverted fluorescence microscope IX83 (Olympus). The establishment of the setup was done using a polychrome light source (Till Photonics) under the control of Visiview Software as previously described (Swain et al. 2016). The microscope was equipped with a cellVivo (Pecon) chamber to control the temperature $\left(37^{\circ} \mathrm{C}\right)$. Prior to measurements, transgenic HFFs and HES2-CMs expressing the Grx1-roGFP2 sensor were washed and loaded with $300 \mu$ l of imaging buffer (with $1 \mathrm{mmol} / \mathrm{L} \mathrm{Ca}^{2+}$ for HES-CMs and $2 \mathrm{mmol} / \mathrm{L} \mathrm{Ca}^{2+}$ for HFFs; see Appendix 1). The cells were excited at $405 \mathrm{~nm}$ and $488 \mathrm{~nm}$ and emission was detected via a CCD camera at $510 \mathrm{~nm}$ (Figure 10). 


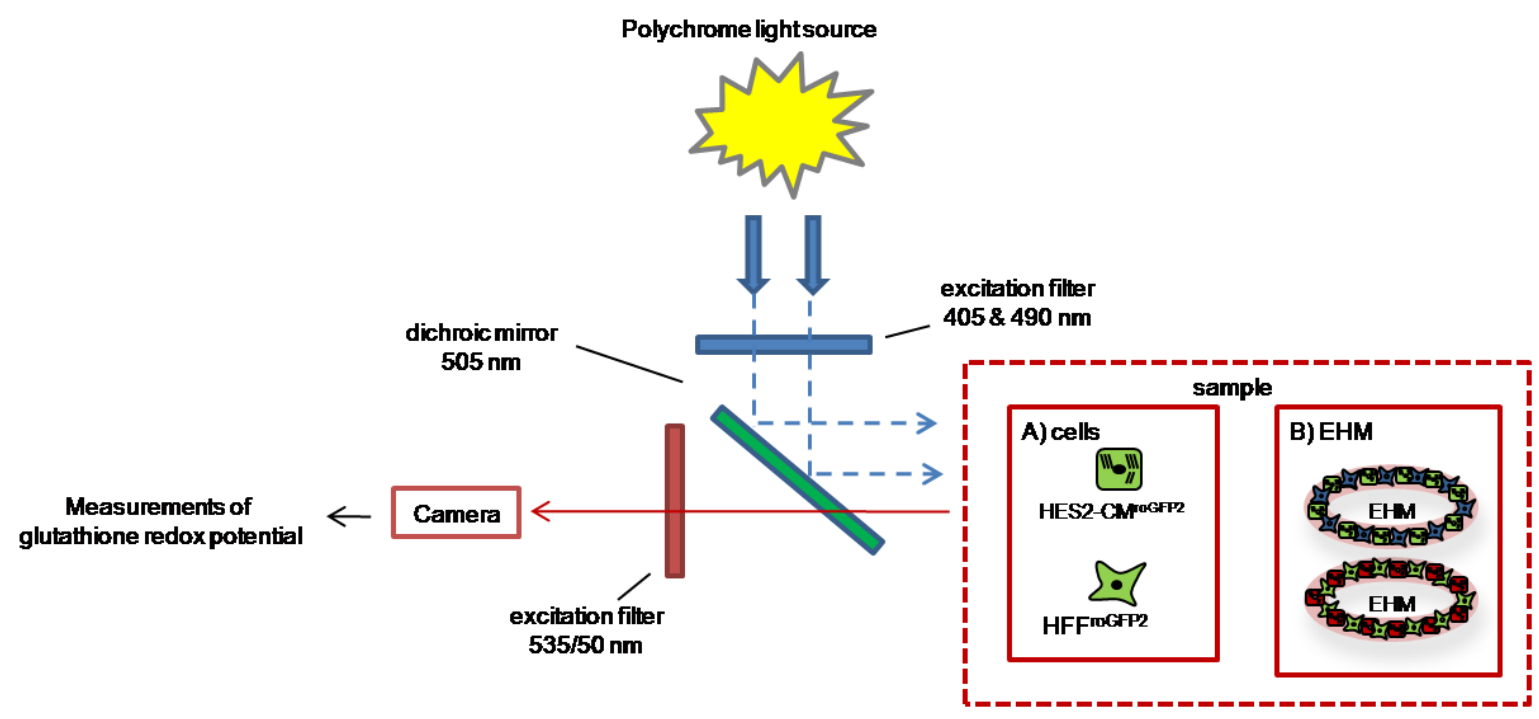

Figure 10: Schematics of the fluorescence capture system used to measure redox changes in monolayer (transgenic HES2-CM and HFFs) and tissue (EHMs). Image adapted from (Swain et al. 2016).

The pseudocolors for 405 and $488 \mathrm{~nm}$ signals were labelled blue and green, respectively. This could help to visualize the redox states of the cells during exposure to oxidants or antioxidant easily. Signal-to-noise ratio was calculated at $60 \mathrm{sec}$ and $180 \mathrm{sec}$ exposure and images were acquired every $5 \mathrm{sec}$ for HFFs and $10 \mathrm{sec}$ for HES2-CMs. Once a stable baseline 405/488 nm ratio was reached (at $60 \mathrm{sec}$ for HFFs and at $180 \mathrm{sec}$ for HES2-CMs), the cells were challenged with oxidants such as hydrogen peroxide $\left(\mathrm{H}_{2} \mathrm{O}_{2}: 0.1-1,000 \mu \mathrm{mol} / \mathrm{L}\right.$; Sigma-Aldrich, \#7722-84-1) or Diamide (DA: 1-1,000 $\mu \mathrm{mol} / \mathrm{L}$; Sigma-Aldrich,\#10465-78-8) and antioxidants such as dithiothreitol (DTT: 0.1-1 mmol/L; Sigma-Aldrich, \#3483-12-3). The maximum oxidation and maximum reduction responses were used to calculate the reduction potential $\mathrm{E}_{\mathrm{GSH}}$ and dynamic range of the roGFP2 sensor in both HFFs and HES2-CM. 


\subsubsection{Calculation of $\mathrm{E}_{\mathrm{rOGFP2}}$ redox potentials}

The calculation of redox potential $E_{\text {roGFP2 }}$ was performed by applying the Nernst Equation and assuming that $\mathrm{E}_{\mathrm{roGFP} 2}$ and $\mathrm{E}_{\mathrm{GSH}}$ are in equilibrium (Meyer and Dick 2010, Morgan et al. 2011):

$$
\text { (1) } E_{G S H}=E_{G S H}^{\circ}-\frac{\mathrm{RT}}{2 \mathrm{~F}} \ln \left(\frac{[G S H] x[G S H]}{[G S S G]}\right)=E_{\text {roGFP } 2}
$$

$R$ is the gas constant $(8.315 \mathrm{~J} / \mathrm{Kmol}), T$ is absolute temperature $(298.15 \mathrm{~K})$ and $F$ is the Faraday's constant $(96.485 \mathrm{C} / \mathrm{mol})$.

Before calculating the redox potential $\mathrm{E}_{\mathrm{GSH}}$, the degree of oxidation of the biosensor (OxD roGFP2) had to be defined (2). OxD roGFP2 was calculated based on the fluorescence intensities recorded at 405 and $488 \mathrm{~nm}$ excitation under reduced (DTT 0.1-1 $\mathrm{mmol} / \mathrm{L})$ and oxidized $\left(\mathrm{H}_{2} \mathrm{O}_{2}-0.1-1,000 \mu \mathrm{mol} / \mathrm{L} ; \mathrm{DA}-1-1,000 \mu \mathrm{mol} / \mathrm{L}\right)$ conditions:

$$
\text { (2) } O x D_{\text {roGFP2 }}=\frac{[\text { roGFP2 }] \mathrm{ox}}{([\text { roGFP2] } \mathrm{red}+[\text { roGFP2] ox })}
$$

The equation (2) can be transformed as below (3):

$$
\text { (3) } O x D_{\text {roGFP2 }}=\frac{\mathrm{I} 405 \times \mathrm{I} 488 \mathrm{red}-\mathrm{I} 405 \mathrm{red} \times \mathrm{I} 488}{\mathrm{I} 405 \times \mathrm{I} \text { I488red-I405 x I4880x + I405ox x I488-I405red x I488 }}
$$

$I$ is the fluorescence intensity at $510 \mathrm{~nm}$ under the indicated excitation wavelengths at maximally oxidized (ox) or reduced (red) conditions.

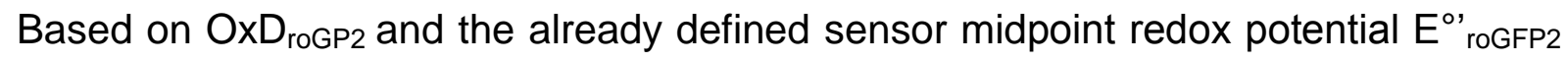
(-280 mV; (Dooley et al. 2004)) the redox potential EroGFP2 can be calculated (4):

$$
\text { (4) } E_{\text {roGFP2 }}=E_{\text {roGFP } 2}^{\circ}-\frac{\mathrm{RT}}{2 \mathrm{~F}} \ln \left(\frac{1-\text { OxDroGFP } 2}{\text { OxDroGFP2 }}\right)
$$


The dynamic range of the biosensor was determined by considering the maximal $\mathrm{H}_{2} \mathrm{O}_{2}$ and DTT responses. Microscope settings (excitation light intensities and exposure times) were all standardized before the measurements.

\subsection{Redox response to reference compounds}

Both transgenic HFFs and HES2-CM were treated with angiotensin II $(0.1-1,000$ nmol/L; Sigma-Aldrich, \#4474-91-3), levosimendan (LEVO - 0.1-10 $\mu \mathrm{mol} / \mathrm{L}$; SigmaAldrich, \#141505-33-1) and omecamtiv mecarbil (OME/CK-1827452 - 0.1-10 $\mu \mathrm{mol} / \mathrm{L}$; AdooQ Bioscience, \#A11206) to study potential effects on glutathione redox potential. After a stable baseline recording for $300 \mathrm{sec}, 300 \mu \mathrm{l}$ of each of the compounds at the specific concentrations were added onto HFFs and HES2-CMs (see Appendix 1). Fluorescence signals were recorded over $900 \mathrm{sec}$. The change in oxidation or reduction response were analysed using Visiview Software.

\subsection{Oxidized peroxiredoxin in fibroblasts and cardiomyocyte}

Oxidation at the protein level was studied after challenging HFF and HES2-CM with $\mathrm{H}_{2} \mathrm{O}_{2}(1-1,000 \mu \mathrm{mol} / \mathrm{L})$ for $1 \mathrm{hr}$. Protein isolation and quantification are explained below.

\subsubsection{Protein isolation}

HFF and HES2-CM were seeded ( $5 \times 10^{5} /$ well) on 6 -well plates and then incubated with $\mathrm{H}_{2} \mathrm{O}_{2}$ for $1 \mathrm{hr}$. Subsequently, the cell lysates were extracted by exposing the cells to CytoBuster ${ }^{\mathrm{TM}}$ Protein extraction reagent (Millipore, 71009; $200 \mu \mathrm{l} / \mathrm{well}$ ) which was mixed with Protease (Roche), Phosphatase inhibitor (PhosSTOP) cocktails (Roche) and Maleimide (Sigma-Aldrich, \#129585; $100 \mathrm{mmol} / \mathrm{L}$ final concentration) for 5 mins at $4{ }^{\circ} \mathrm{C}$. The cell lysates were scraped of the culture dish and collected into separate $2 \mathrm{ml}$ PP tubes. After centrifugation at $14,000 \times \mathrm{g}$ for 5 mins at $4{ }^{\circ} \mathrm{C}$ the supernatant was collected into new PP tubes to either be used directly for immunoblotting or to be stored at $-20^{\circ} \mathrm{C}$ for further applications and analysis. 


\subsubsection{Protein quantification via Bradford Assay}

Protein concentration of the extracted protein samples was determined by performing Bradford protein assay (Bradford 1976). A protein standard curve was analysed after loading defined amounts of $\operatorname{BSA}\left(0,1,2,4,8,16 \mu \mathrm{g}\right.$ in $\left.50 \mu \mathrm{l} \mathrm{H}_{2} \mathrm{O}\right) .2 \mu \mathrm{l}$ of each of the protein samples was diluted with $48 \mu \mathrm{H}_{2} \mathrm{O}$ and analysed in parallel. All samples were analysed in triplicates. $200 \mu \mathrm{l}$ of 1x Bradford reagent (Roti Quant, K015.3, ROTH; diluted in $\mathrm{H}_{2} \mathrm{O}$ ) was added and the samples incubated for 5 mins at RT. Sample absorbance was measured at $595 \mathrm{~nm}$ in a Flexstation ${ }^{\circledR} 3$ Multi-mode microplate reader (Molecular Devices). The concentration of protein samples were analysed and calculated according to the standard curve.

\subsubsection{SDS-PAGE and immunoblotting analysis}

Sodium dodecyl sulphate-polyacrylamide gel electrophoresis (SDS-PAGE) was performed for protein separation according to their molecular weight. $12 \%$ SDSpolyacrylamide gels (Appendix 1) were used. $20 \mu \mathrm{g}$ of each protein sample was mixed with 6x Laemmli Loading Buffer (Appendix 1) together with Maleimide (SigmaAldrich, \#129585; $100 \mathrm{mmol} / \mathrm{L}$ final concentration) to protect the oxidized states of the proteins. The protein samples were denatured at $95^{\circ} \mathrm{C}$ for 5 mins. After denaturation, 30-40 $\mu \mathrm{l}$ of each of the protein samples and 5-10 $\mu \mathrm{l}$ of the Precision Plus ${ }^{\mathrm{TM}}$ Protein Kaleidoscope ${ }^{\mathrm{TM}}$ protein ladder (BioRad) were loaded on the SDS-PAGE gel. The system was operated in $1 \mathrm{x}$ running buffer (Appendix 1) at $70 \mathrm{~V}$ and later the voltage was increased to $120 \mathrm{~V}$ to separate the protein samples.

The proteins were then transferred electrophoretically from the SDS-PAGE gels to polyvinylidene fluoride membranes (PVDF; Amersham ${ }^{\mathrm{TM}} \mathrm{Hybond}^{\mathrm{TM}} \mathrm{P}$ 0.45) in $1 \mathrm{x}$ transfer buffer (Appendix 1) at $4{ }^{\circ} \mathrm{C}$ for $1 \mathrm{hr}$ at $100 \mathrm{~V}$. The membranes were incubated in 10\% Roti-blocking buffer (Roth), which was diluted in distilled $\mathrm{H}_{2} \mathrm{O}$. During incubation, the membranes were placed on a mechanical rocking platform for $1 \mathrm{hr}$ at RT. The primary antibody (anti-peroxiredoxin) was diluted in TBST buffer (Appendix 1; Table 8) and added to the membranes overnight at $4^{\circ} \mathrm{C}$. The next day, the membranes were washed 3x with TBST buffer in 10 mins intervals. A secondary horse radish peroxidase ( $\mathrm{HRP}$ )-coupled antibody was added to the membranes and 
incubated for $1 \mathrm{hr}$ at $\mathrm{RT}$ on the rocking platform at the indicated dilution (Table 8). Detection of the protein-antibody complex was done by using the chemiluminescent reagent Super Signal ${ }^{\circledR}$ West Femto Maximum Sensitive Substrate (Thermo Scientific). A ChemDoc ${ }^{\mathrm{TM}}$ MP Imaging System (BioRad) and Image Lab ${ }^{\mathrm{TM}}$ Software v5.1 (BioRad) were used to visualise and analyse the signals.

\begin{tabular}{|c|c|c|c|c|c|}
\hline \multicolumn{4}{|c|}{ List of Antibodies } & \multicolumn{2}{|c|}{ Dilution / amount } \\
\hline & Name & Species & Manufacturer & WB & FACS \\
\hline \multirow{4}{*}{ 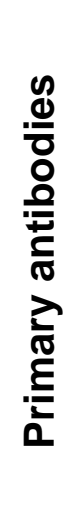 } & $\begin{array}{l}\text { anti-sarcomeric } \\
\alpha \text {-actinin }\end{array}$ & Mouse & $\begin{array}{c}\text { Sigma-Aldrich } \\
(\text { A7811) }\end{array}$ & - & $1: 4,000$ \\
\hline & $\begin{array}{c}\text { anti-Mouse IgG1 } \\
\text { isotype control }\end{array}$ & Mouse & $\begin{array}{c}\text { R\&D Systems } \\
\text { (MAB002) }\end{array}$ & - & $1: 160$ \\
\hline & $\begin{array}{c}\text { Anti- } \\
\text { peroxiredoxin- } \\
\mathrm{SO}_{3} \\
\end{array}$ & Rabbit & $\begin{array}{c}\text { Abcam } \\
\text { (Ab16830) }\end{array}$ & $1: 2,000$ & - \\
\hline & anti-GAPDH & Mouse & $\begin{array}{l}\text { Millipore } \\
\text { (MAB374) }\end{array}$ & $1: 1,000$ & - \\
\hline \multirow{3}{*}{ 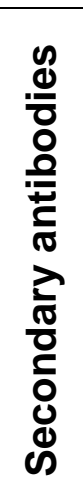 } & $\begin{array}{c}\text { anti-Mouse-Alexa } \\
633\end{array}$ & Goat & $\begin{array}{l}\text { Invitrogen } \\
\text { (A21052) }\end{array}$ & - & $1: 1,000$ \\
\hline & $\begin{array}{l}\text { anti-Mouse } \\
\text { IgG/HRP } \\
\text { conjugate }\end{array}$ & Goat & Dako (P0260) & $1: 10,000$ & - \\
\hline & $\begin{array}{l}\text { anti-Rabbit } \\
\text { IgG/HRP } \\
\text { conjugate }\end{array}$ & Goat & Dako (P0448) & $1: 5,000$ & - \\
\hline 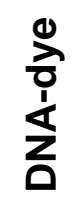 & Hoechst & - & $\begin{array}{c}\text { Invitrogen } \\
\text { (H3570) }\end{array}$ & - & $1 \mu \mathrm{g} / \mathrm{ml}$ \\
\hline
\end{tabular}

Table 8: List of primary/secondary antibodies and fluorescent conjugates used in this study together with the manufacturers (catalogue number) and dilution factors. 


\subsection{Generation and analysis of Human Engineered Heart Muscle}

Engineered heart muscle (EHM) is a hydrogel based (collagen) construct that consists of cellular and ECM components (Soong et al. 2012, Tiburcy et al. 2011, Zimmermann et al. 2002). The EHM used in this study were optimized to exhibit properties of postnatal myocardium (Tiburcy et al. 2017).

\subsubsection{Construction and casting of EHMs}

The EHM reconstitution mixture was cast into custom-made circular molds with central poles (Figure 11A; (Tiburcy et al. 2014)). The outer and inner diameters of an individual circular mold were 10 and $4 \mathrm{~mm}$ (Figure 11B).

A)

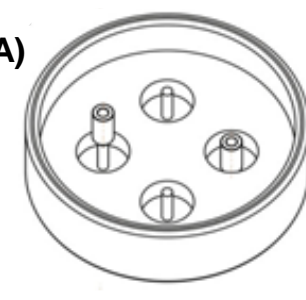

C)

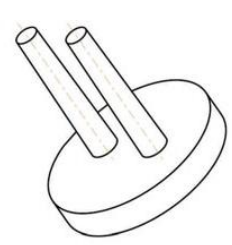

B)

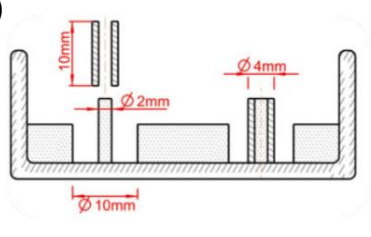

D)

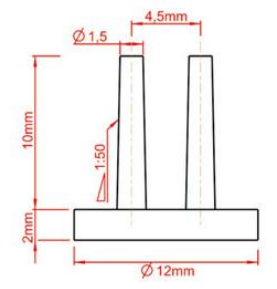

Figure 11: EHM mold and stretcher construct design. (A) Top view of a glass culture dish filled with silicon to form 4 circular molds around central Teflon or silicone cylinder. (B) Side view of the culture dish with two circular casting molds (outer/inner diameter: 10/4 mm). (C-D) Dynamic mechanical silicon stretcher design. Images adapted from (Tiburcy et al. 2014).

Casting of EHMs was done on ice and all master mix components (Table 9) were kept cold $\left(4^{\circ} \mathrm{C}\right)$, under sterile conditions. All steps were done on ice to prevent premature polymerizations. At first, cardiomyocytes and fibroblasts were prepared at a ratio of 70:30, respectively and triturated into EHM medium (Appendix 1). To prepare the master mix, collagen type I was mixed with 2x RPMI (Appendix 1). The 
$\mathrm{pH}$ was adjusted by drop wise addition of $0.1 \mathrm{~N} \mathrm{NaOH}$. A colour change from yellow to pink indicates a $\mathrm{pH}$ of $\sim 7.4$. The cell suspension was then added to the master mix and triturated to ensure homogeneous cell distribution within the EHM mixture. In the last step, $450 \mu \mathrm{l}$ of EHM mixture was added gently into each of the circular molds together with the EHM cell mixture and incubated at $37^{\circ} \mathrm{C}$ with $5 \% \mathrm{CO}_{2}$ for $1 \mathrm{hr}$ to facilitate polymerization.

\begin{tabular}{|l|l|l|l|}
\hline \multirow{2}{*}{ Master mix components } & \multicolumn{3}{|l|}{ Number of EHMs } \\
\cline { 2 - 4 } & $\mathbf{1} \mathbf{x}$ & $\mathbf{4} \mathbf{x}$ & $\mathbf{8} \mathbf{x}$ \\
\hline Bovine Collagen $(6.9 \mathrm{mg} / \mathrm{mL})$ & $68 \mu \mathrm{l}$ & $271 \mu \mathrm{l}$ & $542 \mu \mathrm{l}$ \\
\hline $2 \times \mathrm{RPMl}($ Appendix 1$)$ & $68 \mu \mathrm{l}$ & $271 \mu \mathrm{l}$ & $542 \mu \mathrm{l}$ \\
\hline $0.1 \mathrm{~N} \mathrm{NaOH}$ & $13 \mu \mathrm{l}$ & $53 \mu \mathrm{l}$ & $106 \mu \mathrm{l}$ \\
\hline Cell Suspension $\left(1.45 \times 10^{6} / \mathrm{EHM}\right)$ & $378 \mu \mathrm{l}$ & $1511 \mu \mathrm{l}$ & $3022 \mu \mathrm{l}$ \\
\hline Total volume & $\mathbf{5 2 7} \boldsymbol{\mu l}$ & $\mathbf{2 1 0 6} \boldsymbol{\mu l}$ & $\mathbf{4 2 1 2} \mu \mathrm{l}$ \\
\hline
\end{tabular}

Table 9: EHM master mix components.

After the initial polymerization period, serum-free maturation medium (SFMM) with TGF-B1 (5 ng/mL) was added. The medium was changed after $24 \mathrm{hrs}$. EHMs were left to condense for 3 days and then transferred onto flexible mechanical silicon stretchers (Figure 11C and 11D) in 24-well plates (Figure 12). EHMs culture was for 2 days with medium changes every other day. 


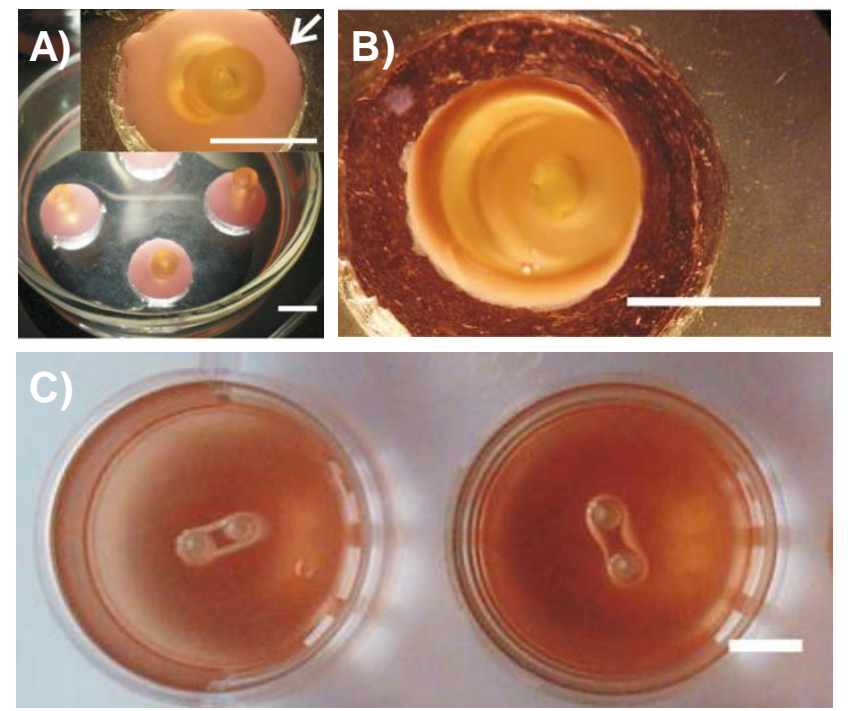

Figure 12: EHM condensation and mechanical (dynamic) loading.(A) EHM condensed for $1 \mathrm{hr}$ at $37^{\circ} \mathrm{C}$. (B) Full condensation of EHM 3 days after casting. (C) Mechanical loading of EHM on dynamic silicon stretchers in 24-well plates. (Bars: 5 mm). Images adapted from (Tiburcy et al. 2014).

\subsubsection{Visualisation of redox changes in transgenic EHMs}

To visualize redox changes, EHMs were cast into three groups: 1) wild type (wt) EHMs with both non-transgenic HES2-CM and HFF; 2) EHMs with transgenic roGFP2-HES2-CM and non-transgenic HFF; and 3) EHM with non-transgenic HFF and transgenic roGFP2-HES2-CM. After 20 days of cultivation, EHMs from each group were washed with pre-warmed $\left(37^{\circ} \mathrm{C}\right)$ Tyrode's solution (Appendix 1) and transferred onto silicon stretchers fixed on a glass coverslip (VWR, \#33386, $25 \mathrm{~mm}$ $\varnothing)$. The imaging setup was composed of a polychrome light source (Till Photonics) under the control of Visiview Software as previously described (Giovarelli et al. 1995, Swain et al. 2016). The EHMs with roGFP2 sensor were excited at $405 \mathrm{~nm}$ and 488 $\mathrm{nm}$ and emission was detected via a CCD camera at $510 \mathrm{~nm}$ (Figure 10). Signal-tonoise ratio was measured at $300 \mathrm{~ms}$ exposure and images were acquired every 10 sec. Once a stable baseline 405/488 nm ratio was reached, generally at $300 \mathrm{sec}$, the EHMs were subjected to Tyrode's solution containing different concentrations of $\mathrm{H}_{2} \mathrm{O}_{2}$ or DTT. The ratiometric glutathione redox changes depending on 405/488 
excitation, were visualized at $4 \mathrm{x}$ magnifications and the mean intensities were used to calculate $\mathrm{OxD}_{\mathrm{rogP2}}$ (see Section 2.4.1).

\subsubsection{Isometric force measurements}

Isometric force measurement were performed as described previously (Zimmermann et al. 2000) in organ baths filled with Tyrode's solution at $37^{\circ} \mathrm{C}$ and perfused with carbogen $\left(95 \% \mathrm{O}_{2}, 5 \% \mathrm{CO}_{2}\right)$ to stabilize the $\mathrm{pH}$ at 7.4 . After 20 days of maturation, the EHMs were transferred onto hooks of a force transducer suspended in organ baths and electrically stimulated at $4 \mathrm{~Hz}$ in an electrical field generated by two parallel platinum electrodes (5 ms monophasic pulses of $200 \mathrm{~mA}$ ). First, EHM were preloaded to a length with optimal force development ( $L_{\max }$; Frank-Starling mechanism) at $1.8 \mathrm{mmol} / \mathrm{L}\left[\mathrm{Ca}^{2+}\right]$. This was followed by thorough washing (2x) in 0.2 $\mathrm{mmol} / \mathrm{L}\left[\mathrm{Ca}^{2+}\right]$ containing Tyrode's solution and finally an exposure to increasing concentrations of $\left[\mathrm{Ca}^{2+}\right](0.2-4 \mathrm{mmol} / \mathrm{L})$ to establish a calcium response curve (maximal inotropic effect and $E_{50}$ ). At $E_{50}$ calcium concentrations (wt $\mathrm{EHM}$, $0.65 \pm 0.06, n=50$; roGFP HFF, 0.83 $\pm 0.12, n=37$; roGFP CM, 0.55 $\pm 0.06, n=31$ ), EHMs were exposed to different concentrations of $\mathrm{H}_{2} \mathrm{O}_{2}(0.1-1,000 \mu \mathrm{mol} / \mathrm{L})$ and DTT $(0.01$ - $3 \mathrm{mmol} / \mathrm{L}$ ) and the maximum and minimum forces were acquired by BMON and analysed by AMON Softwares (G. Jaeckel, Hanau).

\subsubsection{EHM dissociation}

EHM dissociation was performed by incubating EHMs in $1 \mathrm{ml}$ collagenase solution (Appendix 1) for $1 \mathrm{hr}$ at $37^{\circ} \mathrm{C}$. The collagenase solution together with the dissociated parts of EHMs was collected into a PP tube (BD Bioscience) and neutralized with Blocking Buffer Solution (Appendix 1); the remaining EHM fragments were incubated in Accutase Digestion Solution (Appendix 1) for 30 mins at $37^{\circ} \mathrm{C}$ and collected in the same PP tube. After complete dissociation, the cell number and viability were measured by using a CASY Model TT cell counter. The cell suspension was then strained through a $70 \mu \mathrm{m}$ mesh to remove remaining cell/tissue clumps. Subsequently, the cell suspension was either directly used for flow cytometry to measure live cells or fixed in cold $70 \%$ ethanol or $4 \%$ Roti $^{\circledR}$ Histofix at RT for immunofluorescence staining. 


\subsubsection{Immunofluorescent staining and low cytometry}

The cell suspension was centrifuged at $300 \times \mathrm{g}$ for 5 mins to remove the fixing solution. The cells were then incubated in blocking buffer (Appendix 1) for 10 mins on ice. The staining and analysis was as described in section 2.1.4.2.

\subsection{Statistics}

Data are presented as arithmetic mean \pm SEM. Statistical analysis were performed by one-way ANOVA, two-way ANOVA, two-tailed unpaired Student's $t$-test with indicated post hoc tests using GraphPad Prism v6 (GraphPad Software Inc., San Diego). A p-value of $<0.05$ was considered significant. 


\section{Results}

\subsection{Cloning of pGIPZ- Grx1-roGFP2}

The "Grx1-roGFP2" biosensor (Meyer and Dick 2010) was cloned into the pGIPZ backbone for subsequent production of lentivirus particles and transduction to report $\mathrm{E}_{\mathrm{GSH}}$ in cardiomyocytes and fibroblasts.

\subsubsection{Integration of Xbal and Notl cutting sites on CMV-Grx1-roGFP2}

The cytomegalovirus (CMV) promoter together with the biosensor coding sequence (Grx1-roGFP2) from the original plasmid (pLPCX-Grx1-roGFP2; kind gift from T. Dick, Heidelberg) were ligated (Table 1 in Material and Methods) and the sequence size (1,750 base pair) was confirmed by agarose electrophoresis separation (Figure 13A).

Depending on the size ( 1,750 bp), each of the PCR product obtained by the primer combinations (A-D) was ligated to TOPO vector. Rapid ligation of the biosensor into TOPO vector was performed within 5 min incubation due to rapid ligation of the 3'-A overhangs to the PCR product by taq polymerase enzyme. Following overnight (16 hrs) transformation, white (positive) colonies were picked and the modified plasmid was extracted. RE digestion of the plasmids was performed to determine positive clones containing the right ligation orientation by gel electrophoresis (Figure 13B). Clone A containing the ligation of PCR product (CMV-Grx1-roGPF2) of primer combination $\mathrm{A}$ (forward $\mathrm{F} 1$ and reverse $\mathrm{R}$ primers) with TOPO vector, was chosen for further amplification and modification for subcloning into the PGIPZ lentiviral backbone. 
A)

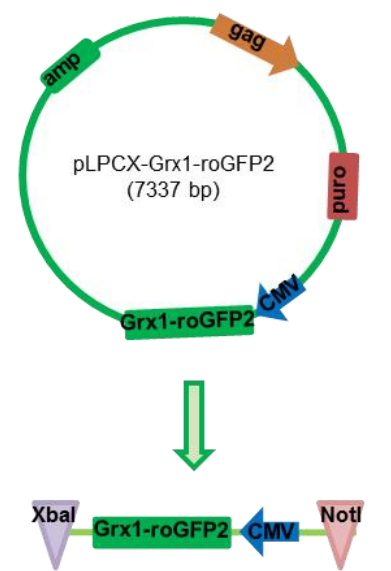

PCR product
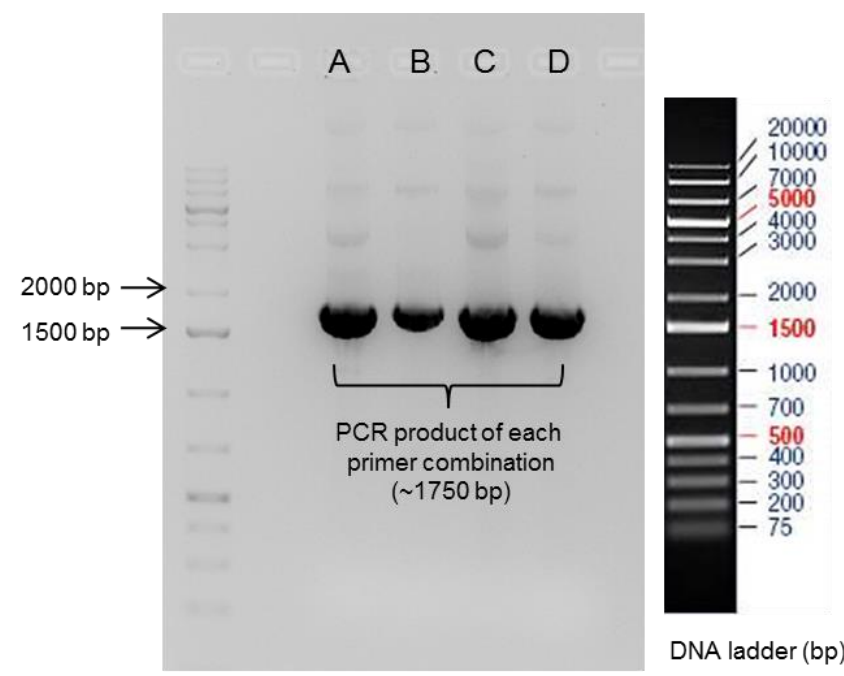

DNA ladder (bp)

B)

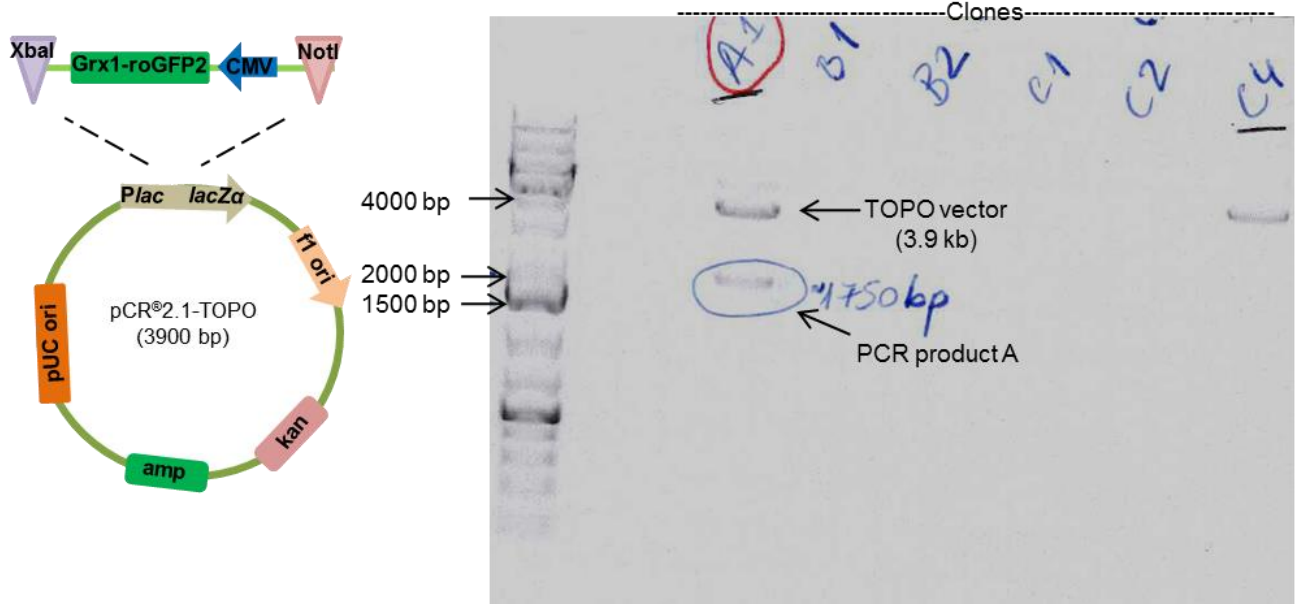

Figure 13: Schematic view of the subcloning strategy. (A) Representative gel of the PCR product for all primer combinations A-D. (B) Schematic diagram of the ligation strategy for the insertion of Grx1-roGFP2 into a TOPO vector and verification of the insertion in clone $\mathrm{A} 1$ by RE digestion (Xbal/Notl). DNA ladder: $1 \mathrm{~kb}$ Plus (Fermentas).

\subsubsection{Generation of the lentivirus plasmid pGIPZ-Grx1-roGFP2}

Generation of pGIPZ-CMV-Grx1-roGFP2 was performed by replacing the CMVturboGFPtag sequence of the pGIPZ backbone with the CMV-Grx1-roGFP2 sequence. The resulting clones were screened by RE digestion (Figure 14A). 23 out of 24 clones were positive for Grx1-roGFP2. Random clones were selected and subjected to DNA sequencing for confirmation of positive ligation of CMV-Grx1- 
roGFP2 construct into pGIPZ plasmid (Figure 14B). Clone 4 was selected for lentivirus production and further glutathione redox measurements.

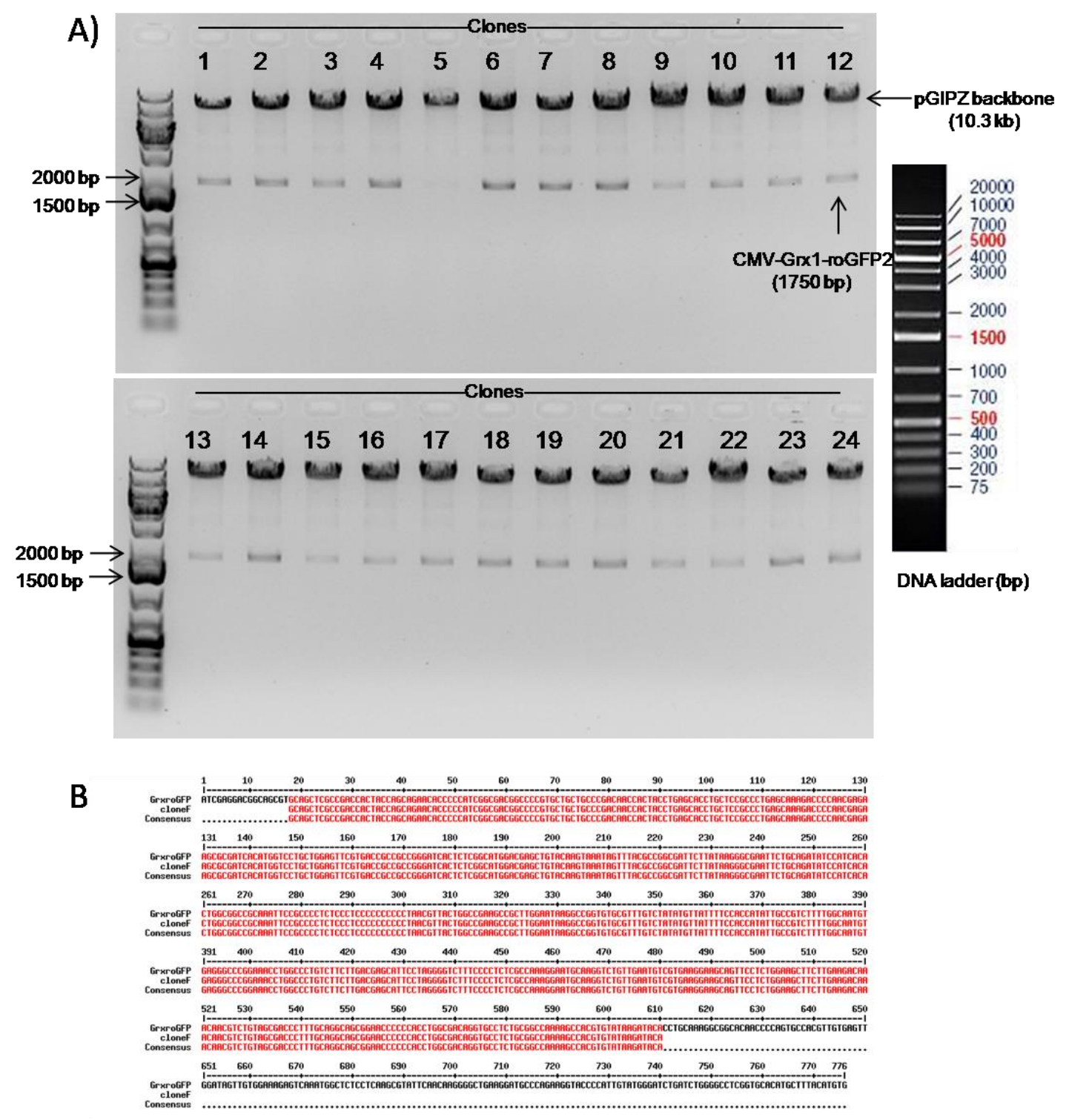

Figure 14: Verification of pGIPZ-CMV-Grx1-roGFP2 cloning. (A) RE digestion with Xbal and Notl released the CMV-Grx1-roGFP2 fragment (1,750 bp) from the vector backbone (10.3 kb). (B) DNA sequencing of Grx1-roGFP2 fragment confirmed its sequence integrity (clone 4). DNA 1 kb Plus Ladder (Fermentas). 


\subsection{Grx1-roGFP2 expression in TSA cells and lentivirus production}

pGIPZ-Grx1-roGFP2 (clones 4 and 7; Figure 14A), the packaging plasmid psPAX.2 and the envelope construct pMD2.G were co-transfected into TSA cells. The original pGIPZ plasmid containing GFP was used as a positive control for TSA cell transfection. After $72 \mathrm{hrs}$ of transfection, TSA cells were visualized for positive GFP expression (Figure 15).

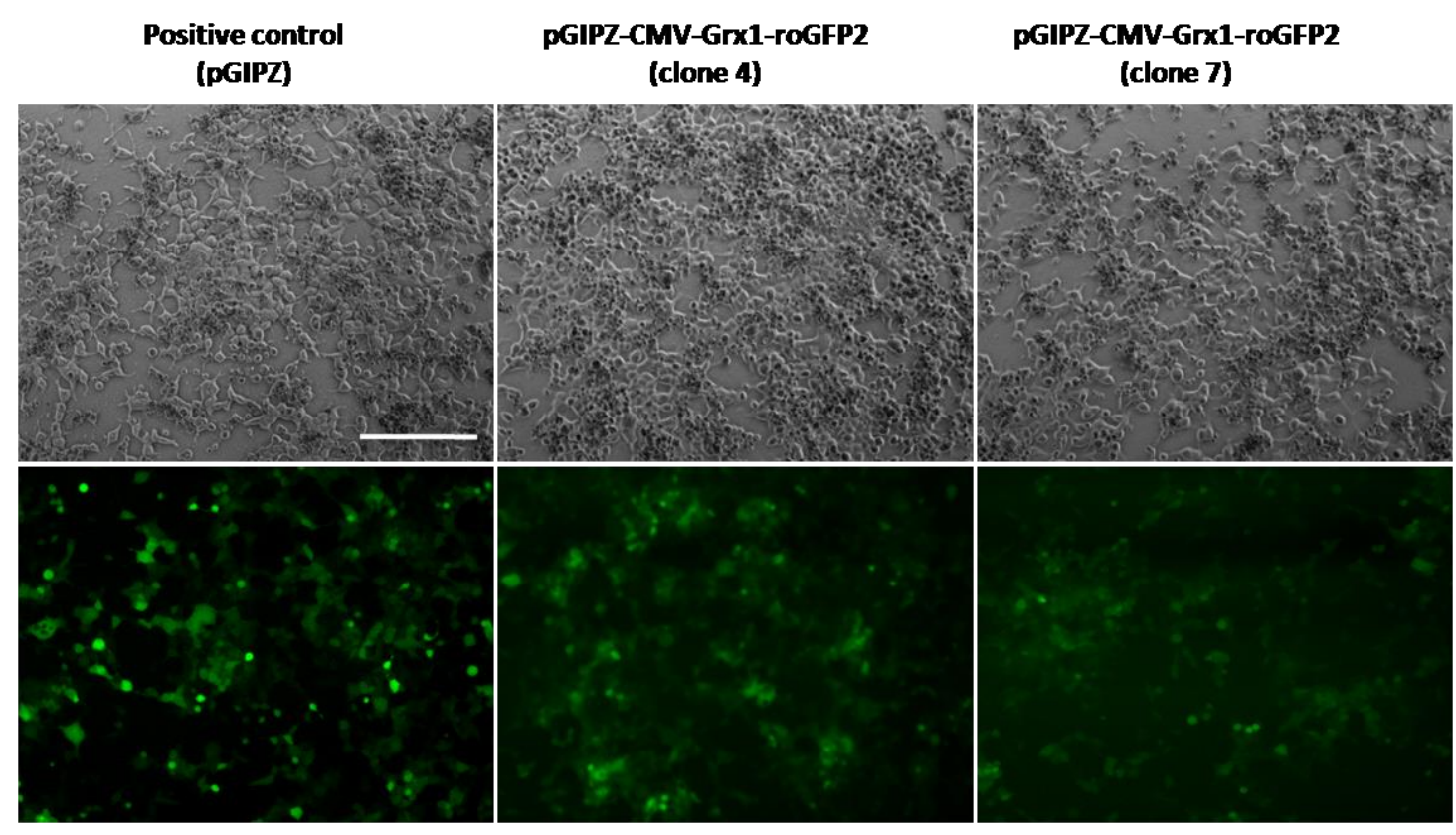

Figure 15: TSA cells after 72 hrs of transfection. (top) Brightfield; (bottom) GFP fluorescence after transfection of pGIPZ-Grx1-ro-GFP2 (clones 4 and 7) and pGIPZ plasmids with the lentivirus helper plasmids psPAX.2 and pMD2.G. Scale bar: 200 $\mu \mathrm{m}$.

Similar GFP signal intensities suggest similar transfection efficiencies in the pGIPZ and pGIPZ-Grx1-roGFP2 (clones 4 and 7) groups. Virus particle containing supernatant was collected and purified before transducing HES2-CM and HFFs. 


\subsubsection{Lentivirus transduction of HFF and HES2-CM}

HFFs were transduced with lentivirus containing the biosensor from both clone 4 and 7. pGIPZ lentivirus transduction was performed as control experiment (Figure 16).

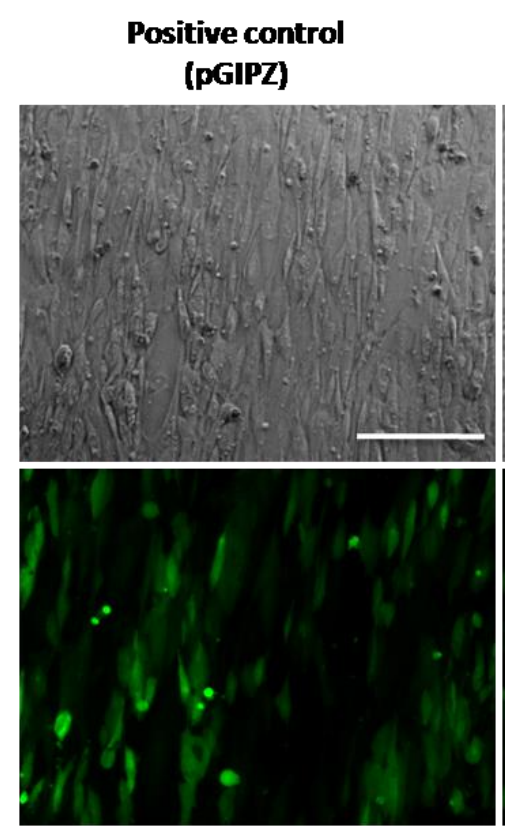

PGIPZ-CMV-Grx1-roGFP2
(clone 4)
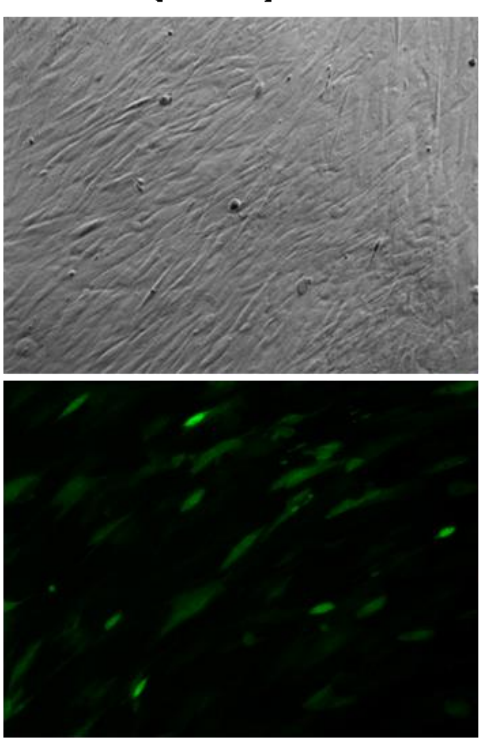

PGIPZ-CMV-Grx1-roGFP2

(clone 7)

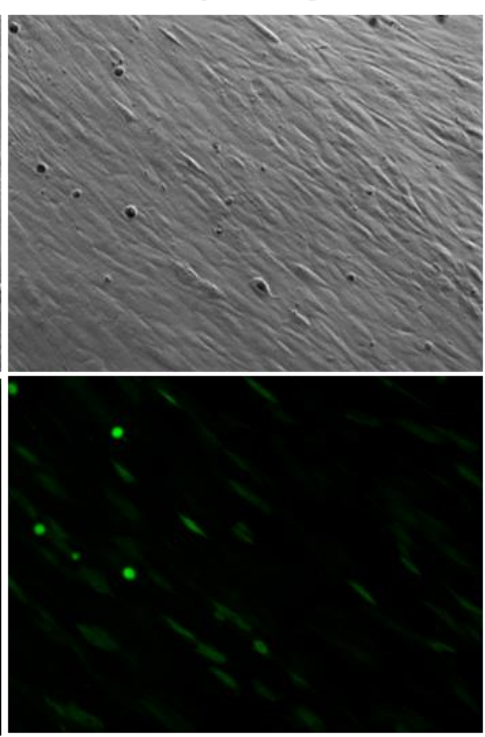

Figure 16: HFFs after transduction. (top) Brightfield; (bottom) GFP fluorescence after transduction with lentivirus harvested from TSA transfected with pGIPZ-Grx1roGFP2 clone 4 and 7 as well as pGIPZ. Scale bar: $200 \mu \mathrm{m}$.

According to previously established killing curves (Soong, PhD thesis) positively transduced HFFs were selected using $1 \mu \mathrm{g} / \mathrm{ml}$ puromycin for 4 days. Lentivirus derived from clone 4 showed a stronger GFP intensity and transduction efficiency than clone 7. As such, all the following experiments were performed using construct 4. Transduction of HFFs and HES2-CMs by the purified lentivirus, resulted in the expression of GFP in each cell type (Figure 17A, 18A). Transduction efficiency was $72 \pm 6.1 \%$ in HFF ( $n=8$; Figure 17B,C) and $47 \pm 7.2 \%$ in HES2-CM ( $n=7$; Figure 18B,C). 
A)

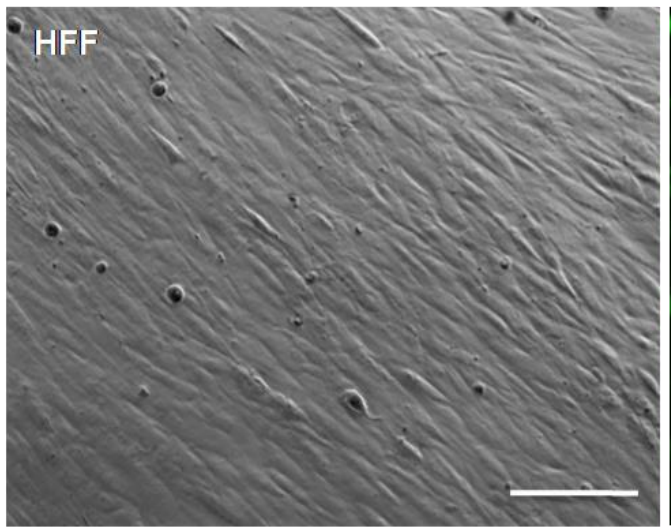

\section{HFF}

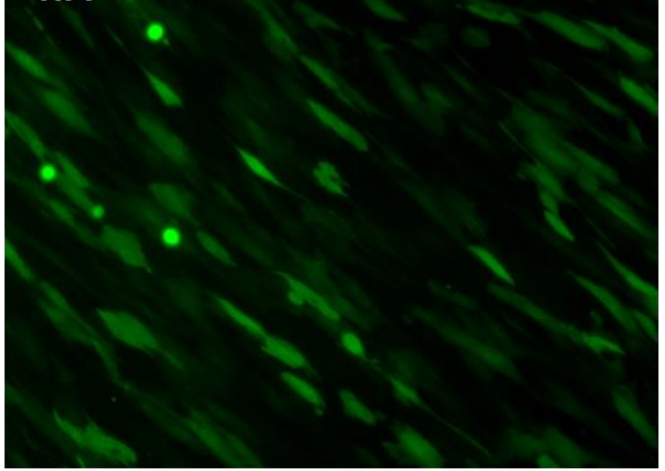

B)

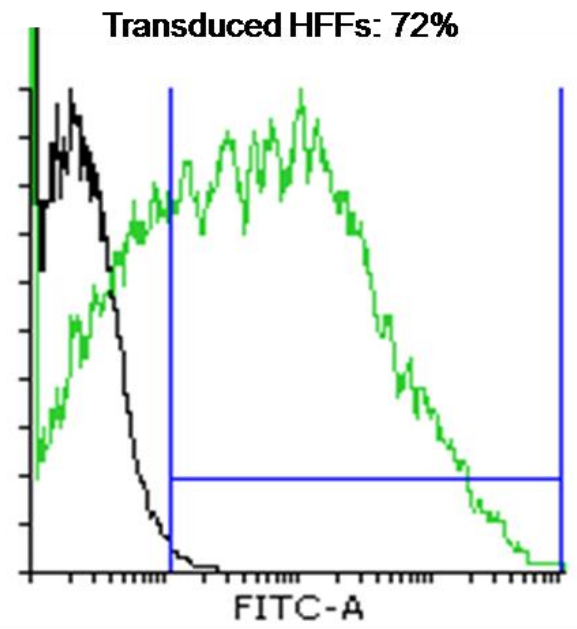

C)

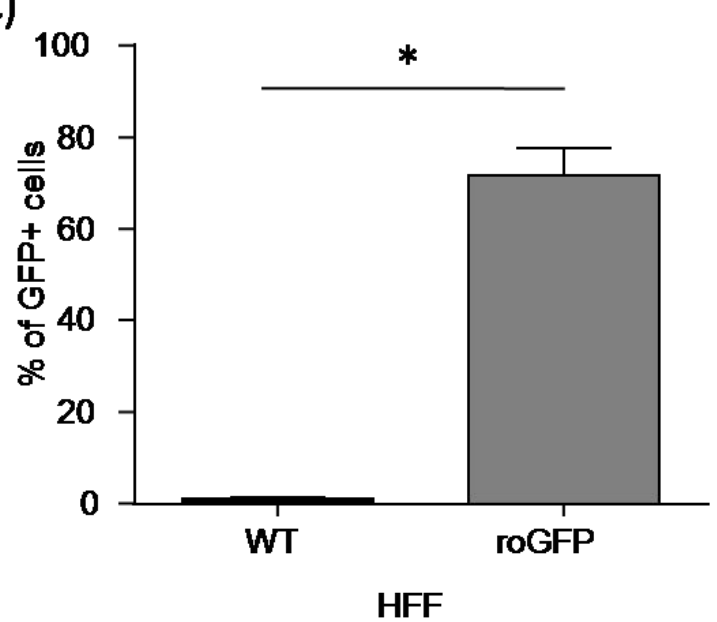

Figure 17: Transduction efficiency in HFFs. (A) (left) Brightfield; (right) GFP fluorescence after lentiviral transduction of HFF with biosensor Grx1-roGFP2. Scale bar: $200 \mu \mathrm{m}$. (B) Transfection efficiency analysed by flow cytometry $(p<0.05$, Student's t-test). 
A)
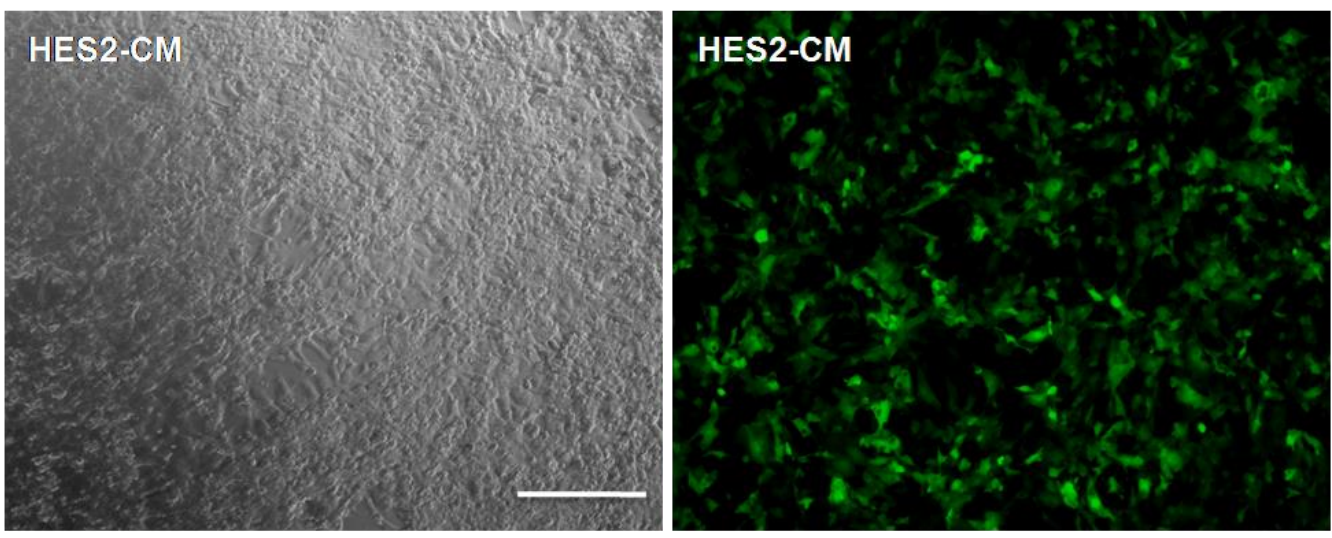

B)

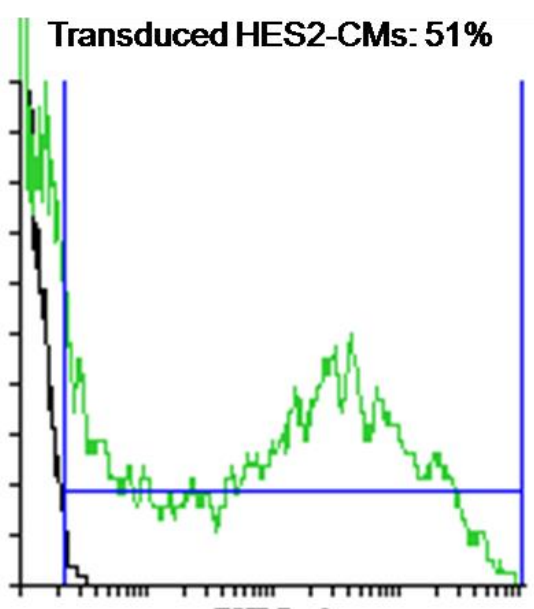

C)

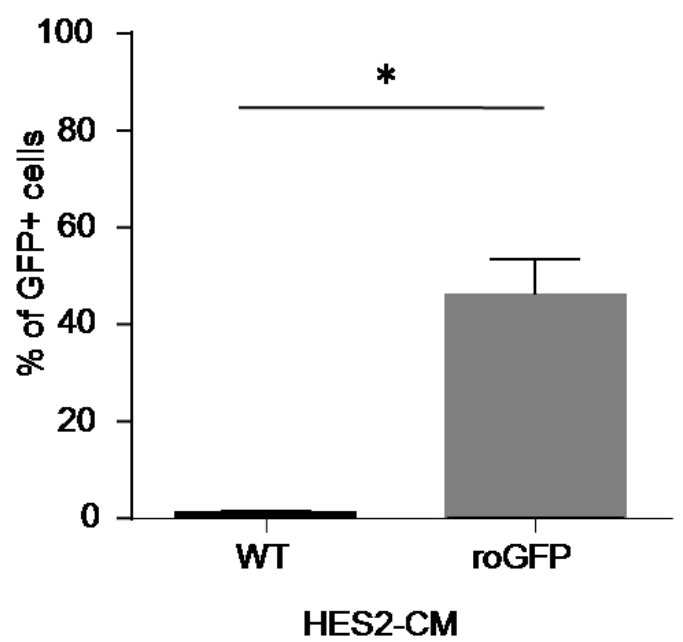

Figure 18: Transduction efficiency in HES2-CMs. (A) (left) Brightfield; (right) GFP fluorescence after lentiviral transduction of HES2-CM with biosensor Grx1-roGFP2. Scale bar: $200 \mu \mathrm{m}$. (B) Transfection efficiency analysed by flow cytometry $(p<0.05$, Student's t-test).

\subsection{Monolayer glutathione redox measurements}

Genetically encoded redox biosensors allow for quantification of redox couples inside the cell. In this study, the glutathione redox sensor Grx1-roGFP2 was expressed in the cytosol. By titration of $\mathrm{H}_{2} \mathrm{O}_{2}$, DA and DTT it was possible to quantify the ratio of the reduced and oxidized forms of the glutathione couple [GSH:GSSG] in HFF and HES2-CM. Fluorescence changes were analysed ratiometrically from regions of interests (ROI), which were manually defined to encompass individual cells (Figure 19). 

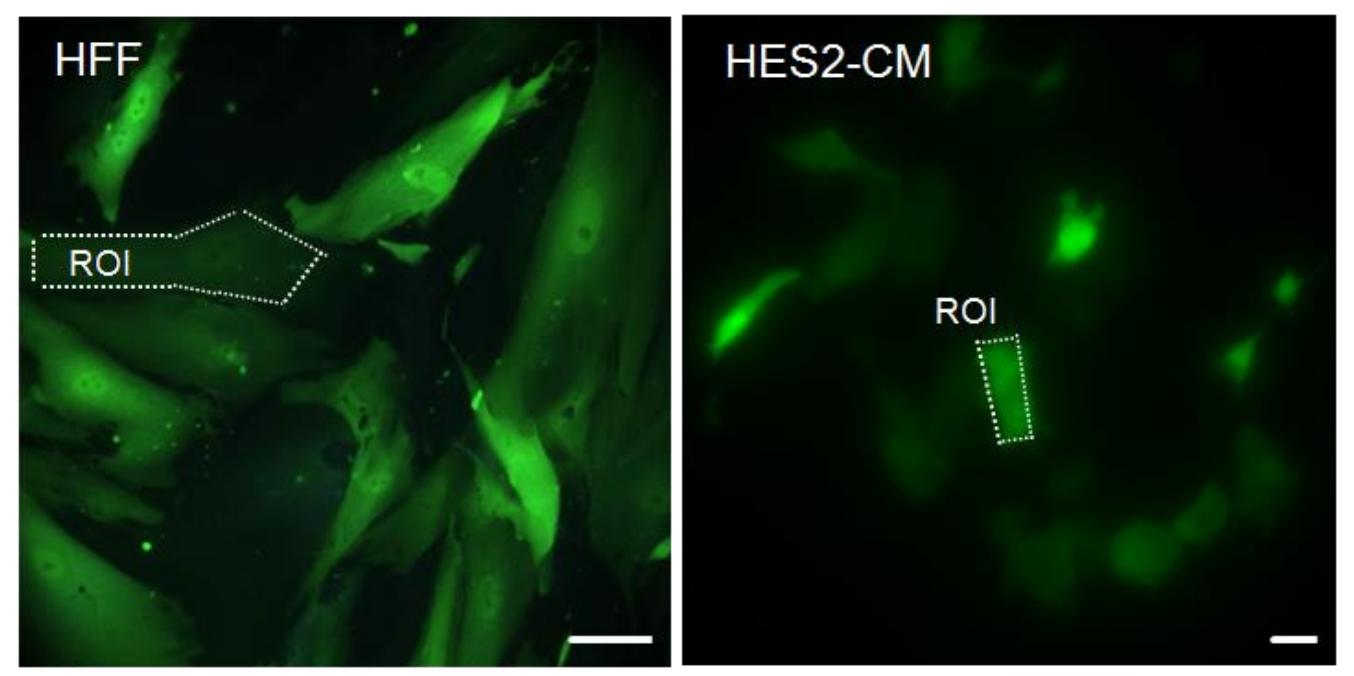

Figure 19: Cell-specific analysis of the glutathione redox sensor in HFF and HES2-CM. ROI: region of interest for fluorescence intensity measurements. Scale bars: 40 and $20 \mu \mathrm{m}$ in HFF and HES2-CM, respectively.

\subsubsection{Oxidative challenge with $\mathrm{H}_{2} \mathrm{O}_{2}$}

Exposure to $\mathrm{H}_{2} \mathrm{O}_{2}$ causes disulfide bond formation between Cys147 and Cys204 in the glutathione protein (GSH + GSH $\rightarrow$ GSSG). This change promotes the protonation of the roGFP2 chromophore, leading to increased fluorescence emission under $405 \mathrm{~nm}$ versus $490 \mathrm{~nm}$ excitation. Treatment with DTT has the reverse function (GSSG $\rightarrow \mathrm{GSH}+\mathrm{GSH}$ ).

Grx1-roGFP2 transduced HFFs (Figure 20) and HES2-CMs (Figure 21) were treated with different concentrations of $\mathrm{H}_{2} \mathrm{O}_{2}(0.1-1,000 \mu \mathrm{mol} / \mathrm{L})$. Interestingly, a concentration dependent response was observed in HFF whereas HES2-CM demonstrated an all or nothing response with maximal effects at $100 \mu \mathrm{mol} / \mathrm{L}$ in $\mathrm{HFF}$ (Figure 20) versus $10 \mu \mathrm{mol} / \mathrm{L}$ in HES2-CM (Figure 21). 

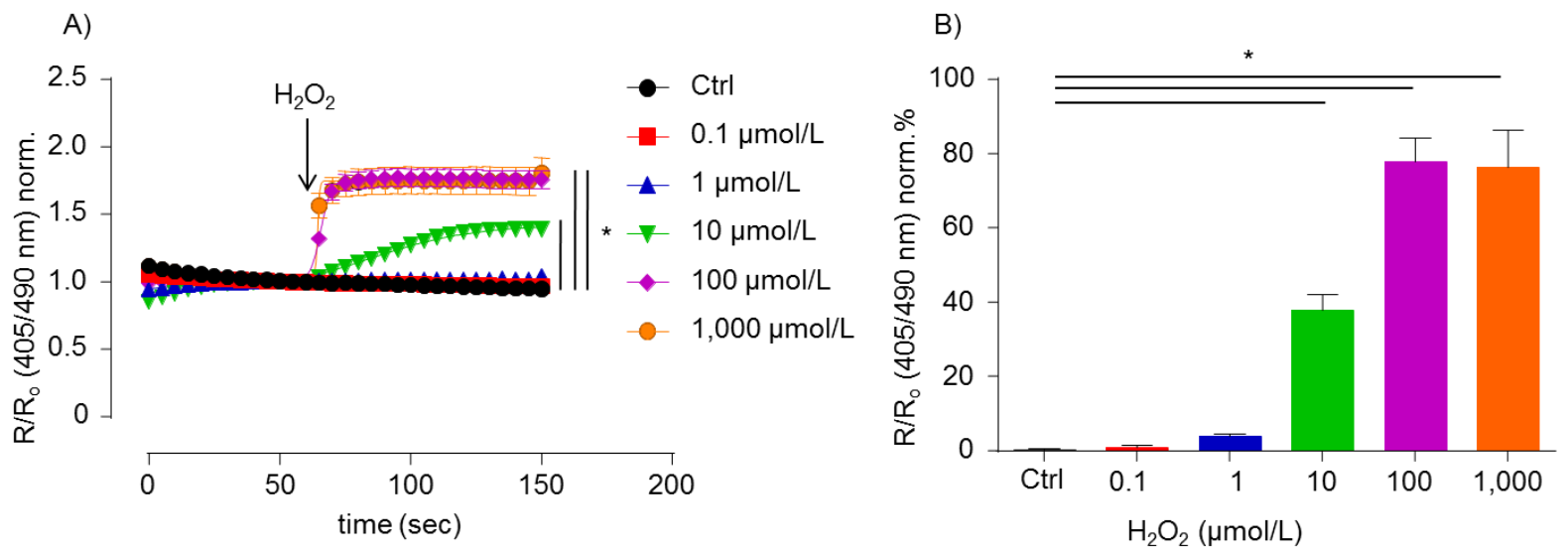

Figure 20: Live imaging of glutathione oxidation in $\mathrm{HFFs}$ under $\mathrm{H}_{2} \mathrm{O}_{2}$ challenge. (A) Changes in the ratiometric fluorescence signal of the Grx1-roGFP2 in HFF in response to $\mathrm{H}_{2} \mathrm{O}_{2}$ at the indicated concentrations as a function of time $\left(\mathrm{R}_{0}=405 / 490\right.$ $\mathrm{nm}$ excited signals at baseline); $\mathrm{H}_{2} \mathrm{O}_{2}$ was added at $60 \mathrm{sec}$ of stable baseline recordings. (B) Maximal ratiometric signal increase at the indicated $\mathrm{H}_{2} \mathrm{O}_{2}$ concentrations. $19-43$ cells/group; ${ }^{*} \mathrm{p}<0.05$ vs. Ctrl by two-way (A) and one-way (B) ANOVA followed by Dunnett's post hoc test.
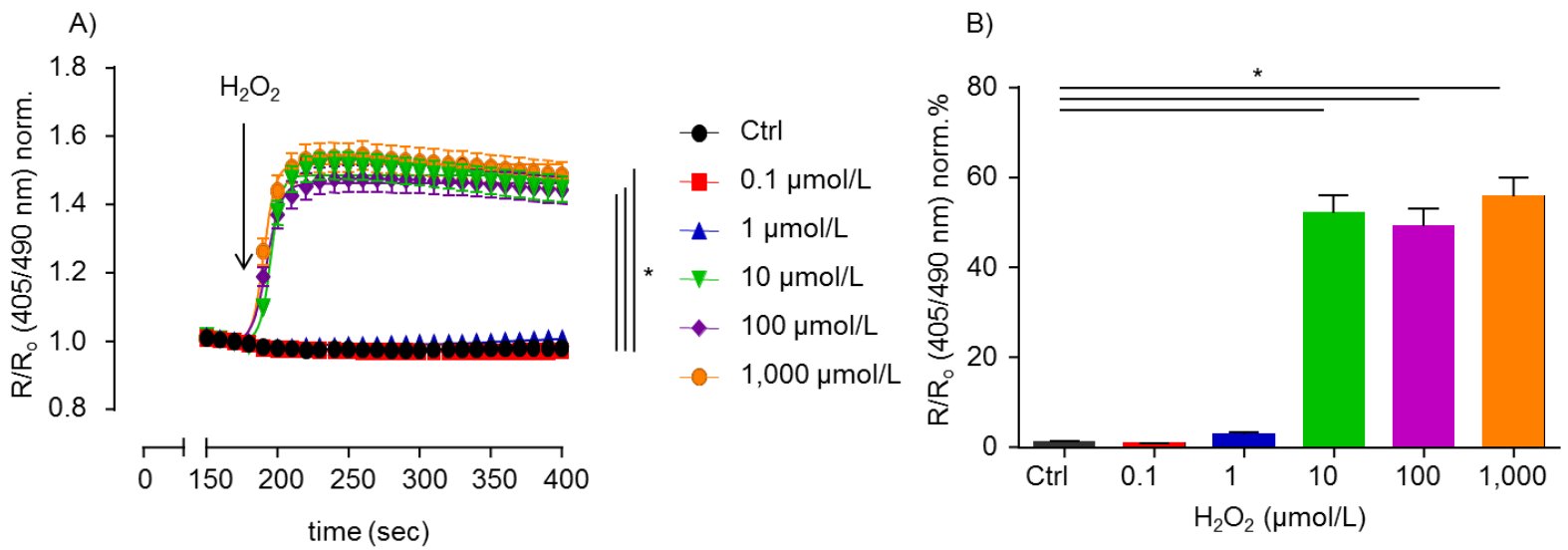

Figure 21: Live imaging of glutathione oxidation in HES2-CMs under $\mathrm{H}_{2} \mathrm{O}_{2}$ challenge. (A) Changes in the ratiometric fluorescence signal of the Grx1-roGFP2 in HES2-CM in response to $\mathrm{H}_{2} \mathrm{O}_{2}$ at the indicated concentrations as a function of time $\left(\mathrm{R}_{0}=405 / 490 \mathrm{~nm}\right.$ excited signals at baseline); $\mathrm{H}_{2} \mathrm{O}_{2}$ was added at $180 \mathrm{sec}$ of stable baseline recordings. (B) Maximal ratiometric signal increase at the indicated $\mathrm{H}_{2} \mathrm{O}_{2}$ concentrations. 46-71 cells/group; ${ }^{*} \mathrm{p}<0.05$ vs. Ctrl by two-way (A) and one-way (B) ANOVA followed by Dunnett's post hoc test. 
The onset of glutathione oxidation was similar in HFF (Figure 22A) and HES2-CM (Figure 22B) at $10 \mu \mathrm{mol} / \mathrm{L}$, but clearly enhanced at higher concentrations in HFF only.

A)

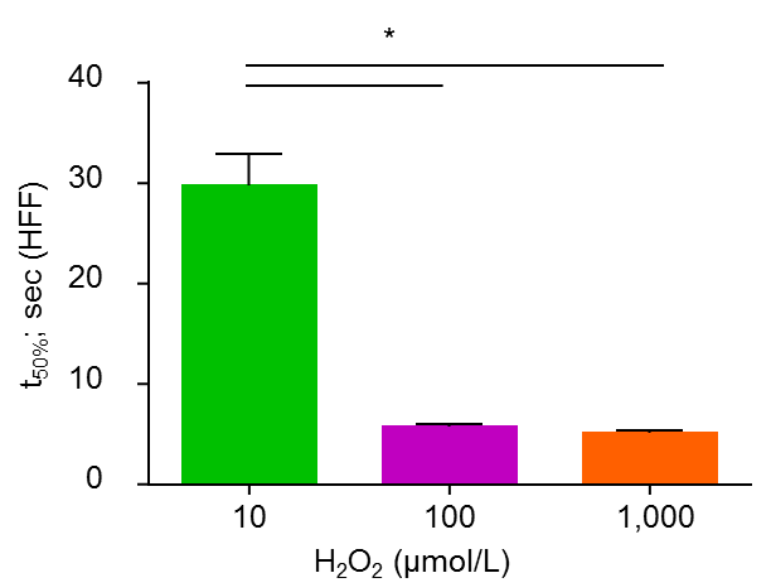

B)

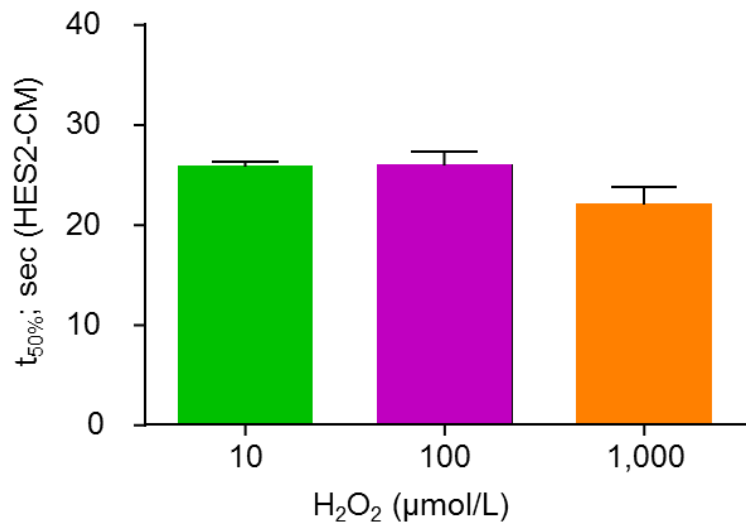

Figure 22: Differences in glutathione oxidation kinetics in HFF and HES2-CM. Time required for half maximum reporter signal $\left(t_{50}\right)$ increase in $\left.\operatorname{HFF}(n=27-41) ; \mathbf{A}\right)$ and HES2-CM ( $n=39-47$ ); B) cultures. ${ }^{*} p<0.05$ vs. $10 \mu \mathrm{mol} / \mathrm{L}_{2} \mathrm{H}_{2}$ by one-way ANOVA followed by Tukey's post hoc test.

\subsubsection{Response of HFF and HES2-CM to DA}

Diamide (DA) is a thiol group oxidant that oxidizes the thiol groups of roGFP2 directly into disulfides. Thus it does not depend on the availability of glutathione in the cell (Swain et al. 2016). Hence, DA stimulation allows for the determination of the maximal roGFP2 signal change obtainable under an oxidative challenge. HFFs and HES2-CMs expressing the Grx1-roGFP2 were exposed to different concentrations of DA (1 - 1,000 $\mu \mathrm{mol} / \mathrm{L})$. Similar as for $\mathrm{H}_{2} \mathrm{O}_{2}$ maximal oxidation under DA was achieved at lower concentrations in HES2-CM as compared to HFF (0.1 vs $1 \mathrm{mmol} / \mathrm{L}$ ) (Figure 23 and 24). Time to maximal reporter signal was similarly DA concentration dependent (Figure 25). 

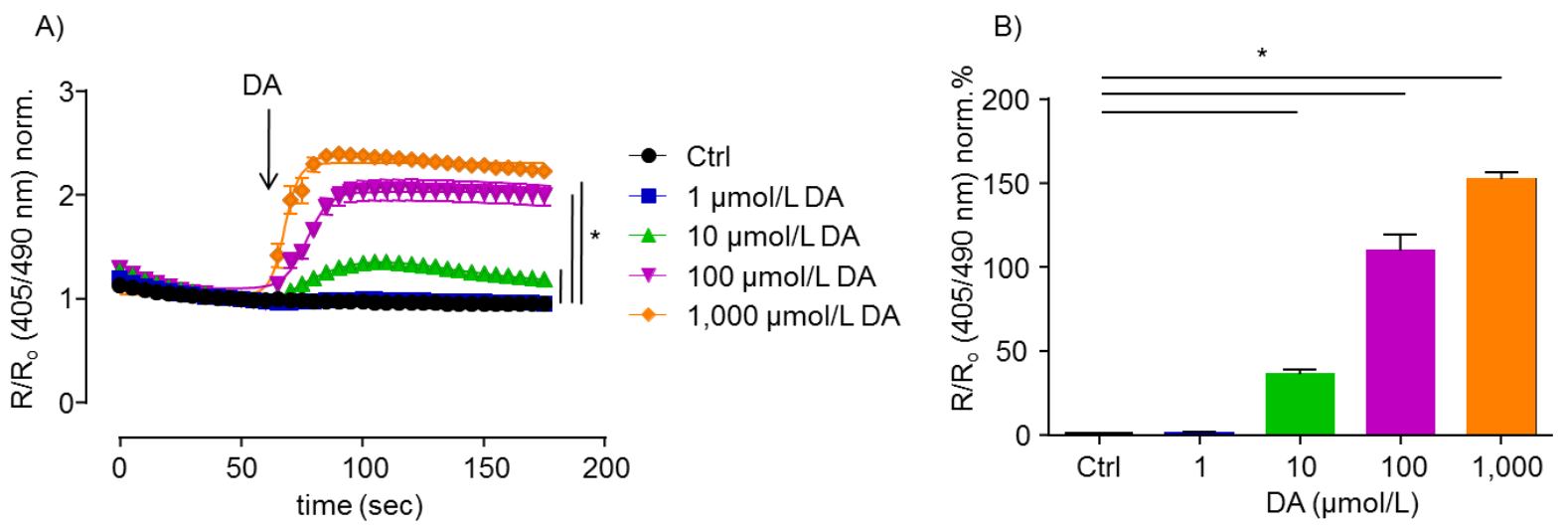

Figure 23: Live imaging of glutathione oxidation in HFFs under DA challenge.

(A) Changes in the ratiometric fluorescence signal of the Grx1-roGFP2 in HFF in response to $D A$ at the indicated concentrations as a function of time $\left(R_{0}=405 / 490\right.$ $\mathrm{nm}$ excited signals at baseline); DA was added at $60 \mathrm{sec}$ of stable baseline recordings. (B) Maximal ratiometric signal increase at the indicated DA concentrations. 26-60 cells/group; ${ }^{*} p<0.05$ vs. Ctrl by two-way (A) and one-way (B) ANOVA followed by Dunnett's post hoc test.
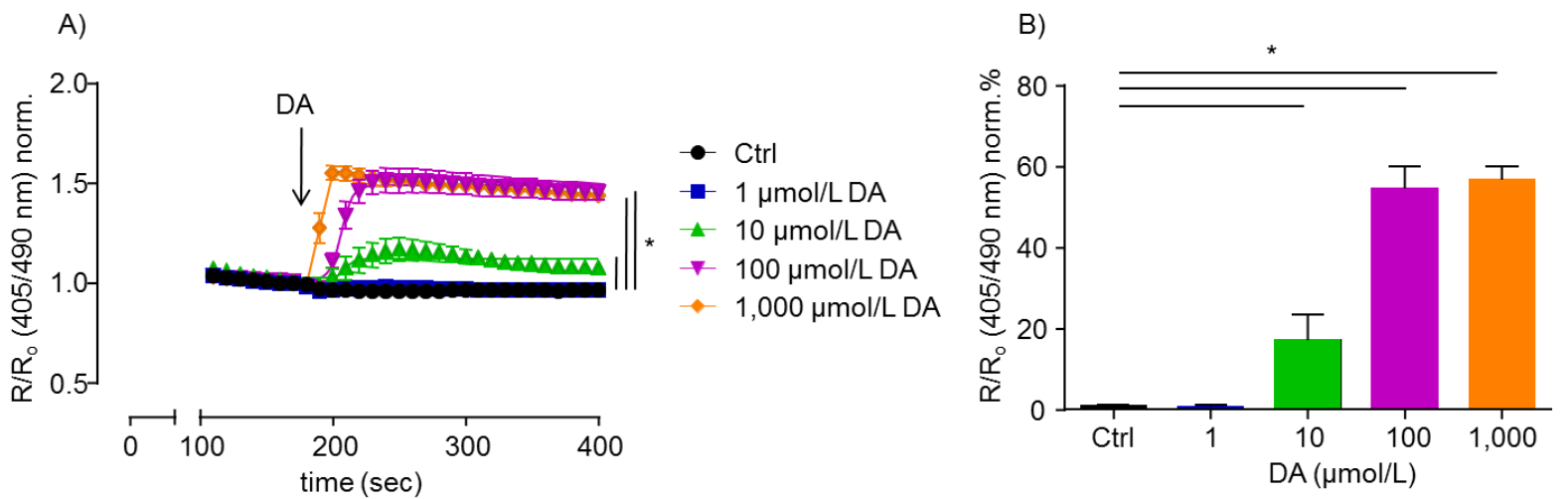

Figure 24: Live imaging of glutathione oxidation in HES2-CMs under DA challenge. (A) Changes in the ratiometric fluorescence signal of the Grx1-roGFP2 in HES2-CM in response to DA at the indicated concentrations as a function of time $\left(R_{0}=405 / 490 \mathrm{~nm}\right.$ excited signals at baseline); DA was added at $180 \mathrm{sec}$ of stable baseline recordings. (B) Maximal ratiometric signal increase at the indicated DA concentrations. 7-9 cells/group; ${ }^{*} p<0.05$ vs. Ctrl by two-way (A) and one-way (B) ANOVA followed by Dunnett's post hoc test. 
The time required for biosensor oxidation in HFFs is similar and independent of the DA concentrations (Figure 25A). Interestingly, HES2-CM showed a DA concentration dependent acceleration in DA mediated oxidation with slower and faster kinetics at 10 and $1,000 \mu \mathrm{mol} / \mathrm{L} \mathrm{DA}$, respectively (Figure 25B).
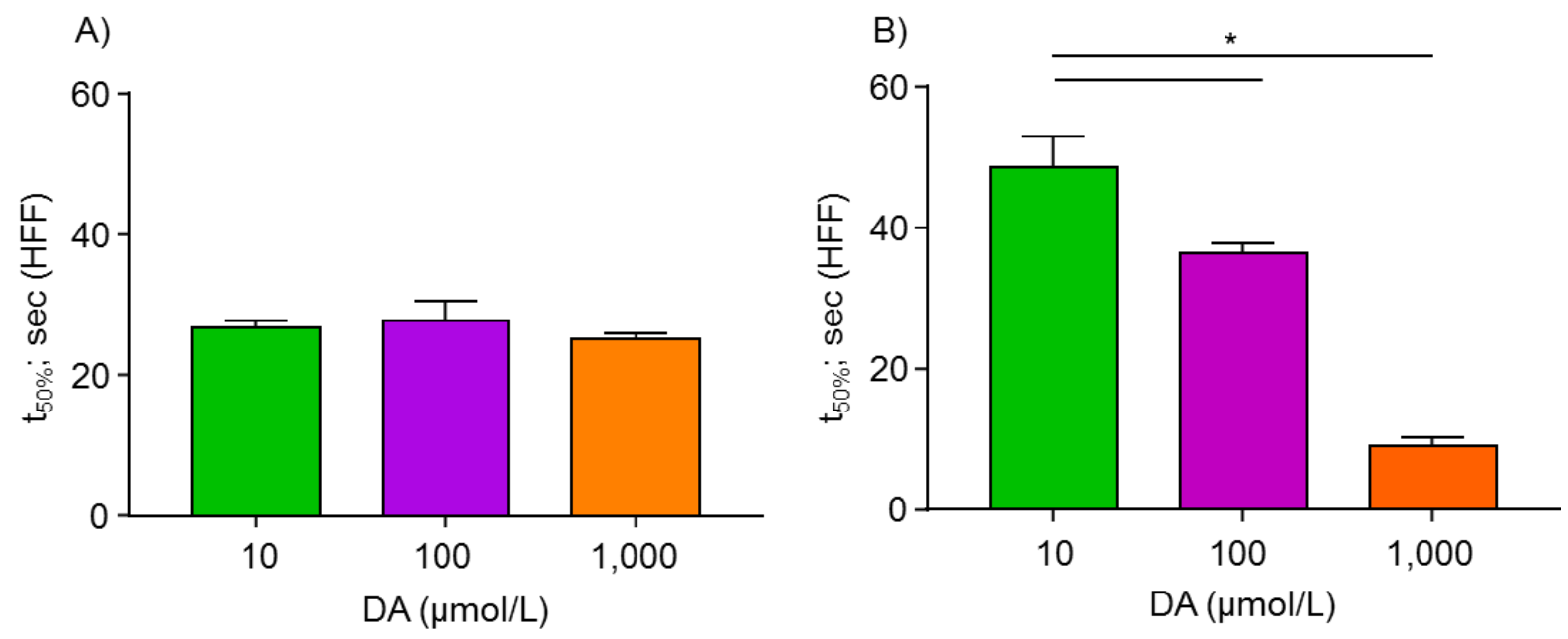

Figure 25: Differences in biosensor oxidation kinetics in HFF and HES2-CM. Time required for half maximum reporter signal $\left(t_{50}\right)$ increase in $\operatorname{HFF}(n=12-55)$; A) and HES2-CM ( $\mathrm{n}=7-15)$; B) cultures. ${ }^{*} \mathrm{p}<0.05$ vs. $10 \mu \mathrm{mol} / \mathrm{L}$ and $100 \mu \mathrm{mol} / \mathrm{L}$ DA by one-way ANOVA followed by Tukey's post hoc test.

\subsubsection{Response of HFF and HES2-CM to DTT}

To investigate the reducing properties of the biosensor Grx1-roGFP2, trangenic HFFs and HES2-CMs were exposed to various concentrations $(0.01-1.0 \mathrm{mmol} / \mathrm{L})$ of reducing agent DTT (Figures 26 and 27). In contrast to the findings in response to oxidation with $\mathrm{H}_{2} \mathrm{O}_{2}$ and DA there was a clear concentration dependency in HFF and HES2-CM. However, HFF demonstrated a much stronger signal reduction by 1 $\mathrm{mmol} / \mathrm{L}(-64 \pm 1.6 \% ; \mathrm{n}=28)$ as compared to the signal reduction in HES2-CM ($22 \pm 0.8 \% ; n=58)$. 

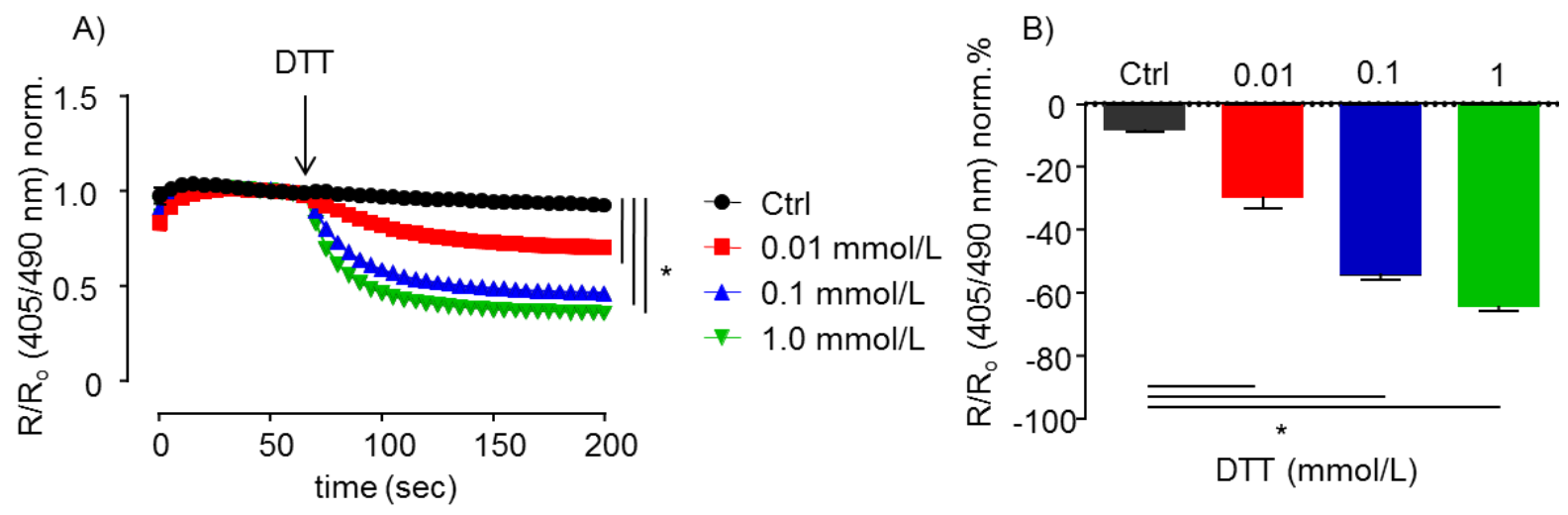

Figure 26: Live imaging of glutathione oxidation in HFF under DTT challenge.

(A) Changes in the ratiometric fluorescence signal of the Grx1-roGFP2 in HFF in response to DTT at the indicated concentrations as a function of time $\left(R_{0}=405 / 490\right.$ $\mathrm{nm}$ excited signals at baseline); DTT was added at $180 \mathrm{sec}$ of stable baseline recordings. (B) Maximal ratiometric signal decrease at the indicated DTT concentrations. 21-37 cells/group; ${ }^{*} p<0.05$ vs. Ctrl by two-way (A) and one-way (B) ANOVA followed by Dunnett's post hoc test.
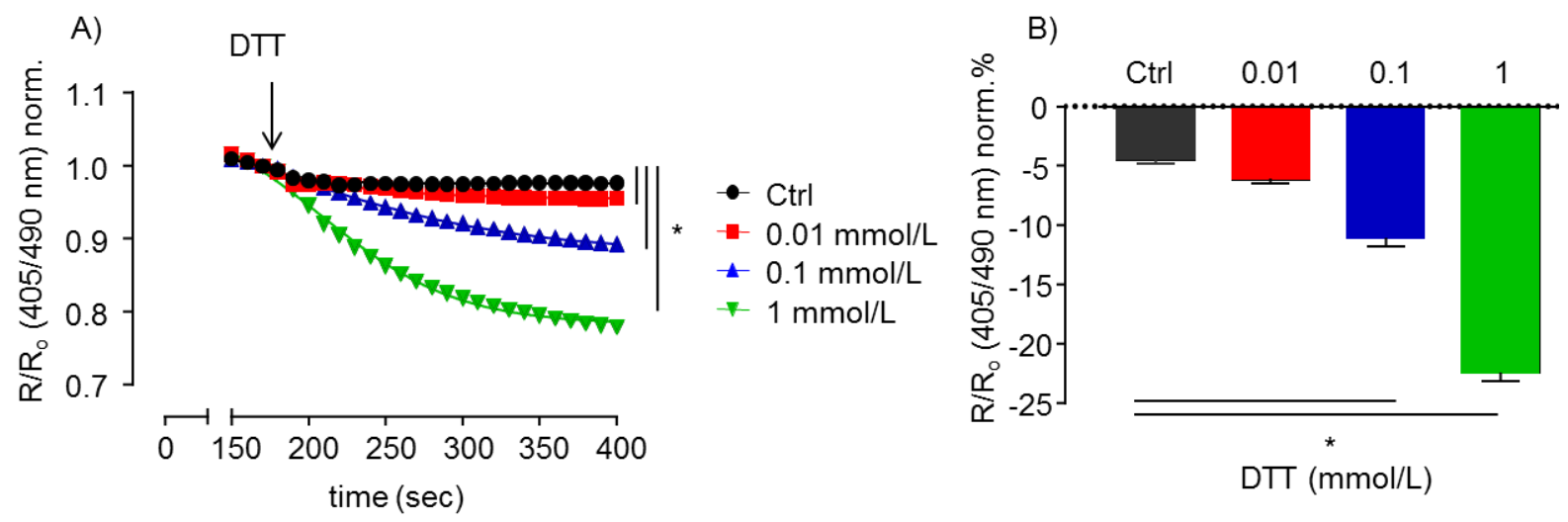

Figure 27: Live imaging of glutathione oxidation in HES2-CMs under DTT challenge. (A) Changes in the ratiometric fluorescence signal of the Grx1-roGFP2 in HES2-CM in response to DTT at the indicated concentrations as a function of time $\left(R_{0}=405 / 490 \mathrm{~nm}\right.$ excited signals at baseline); DTT was added at $180 \mathrm{sec}$ of stable baseline recordings. (B) Maximal ratiometric signal decrease at the indicated DTT concentrations. $46-85$ cells/group; ${ }^{*} p<0.05$ vs. Ctrl by two-way (A) and one-way (B) ANOVA followed by Dunnett's post hoc test. 
Another obvious difference was the markedly slower kinetics of Grx1-roGFP2 reduction $(21 \pm 0.9 \mathrm{~ms}$ in HFF vs. $83 \pm 3.1 \mathrm{~ms}$ in HES2-CM at $1 \mathrm{mmol} / \mathrm{L}$ DTT; $\mathrm{n}=$ 28/58; Figure 28).

A)

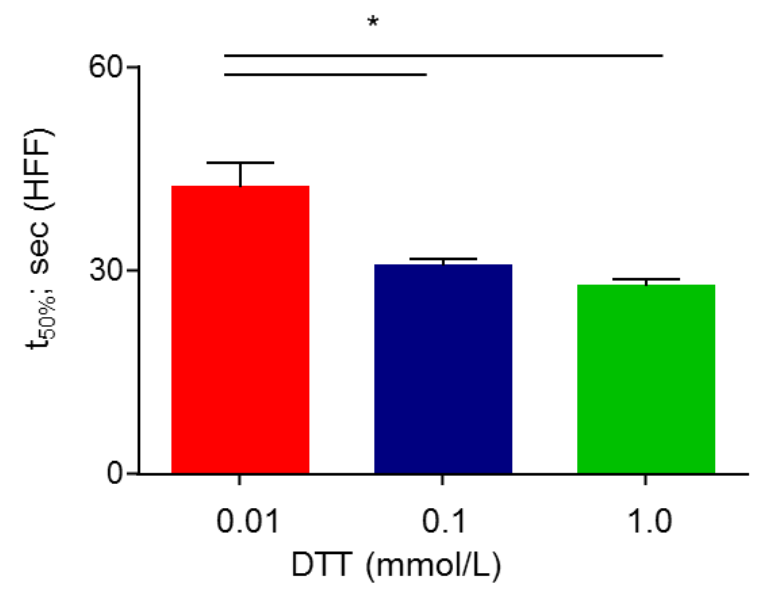

B)

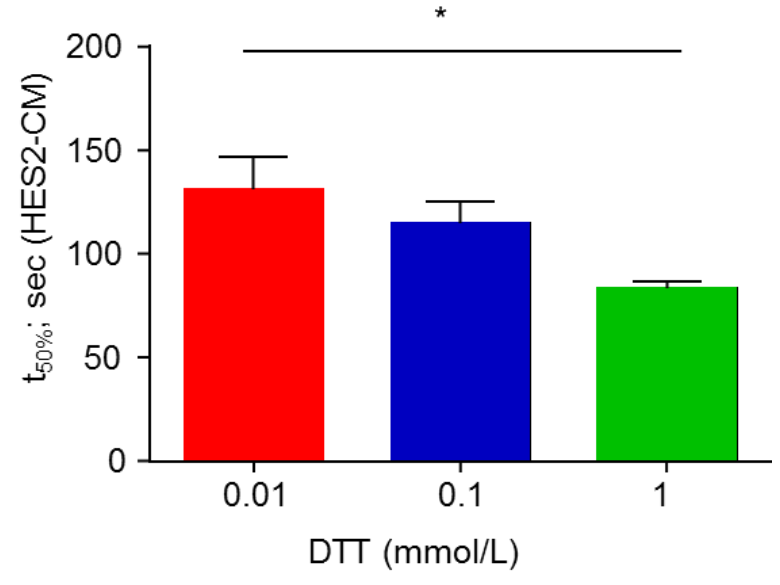

Figure 28: Differences in glutathione reduction kinetics in HFF and HES2-CM.

Time required for half maximum reporter signal $\left(t_{50}\right)$ decrease in $\left.\operatorname{HFF}(n=21-37) ; \mathbf{A}\right)$ and HES2-CM ( $n=40-62)$; B) cultures; * $\mathrm{p}<0.05$ vs. $0.01 \mathrm{mmol} / \mathrm{L}$ DTT by one-way ANOVA followed by Tukey's post hoc test.

\subsubsection{Calculation of the glutathione redox potential}

Glutathione redox potential ( $E_{G S H}$ ) was calculated for both cell types by using the Nernst Equation (Section 2.4.1, equation (3) Materials and Methods). The calculations were based on the $\mathrm{OxD}_{\text {roGFP2 }}$ of the maximum response for oxidation $\left(\mathrm{H}_{2} \mathrm{O}_{2}\right.$ and $\left.\mathrm{DA}\right)$ and the maximum response for reduction (DTT). HES2-CMs demonstrated a significantly more reduced $\mathrm{E}_{\mathrm{GSH}}$ compared to HFFs (Table 10). There was no difference in DA-DTT and $\mathrm{H}_{2} \mathrm{O}_{2}$-DTT signal range based calculation of $\mathrm{E}_{\mathrm{GSH}}$, suggesting that maximal Grx1-roGFP2 sensor oxidation could be achieved with $\mathrm{H}_{2} \mathrm{O}_{2}$. 


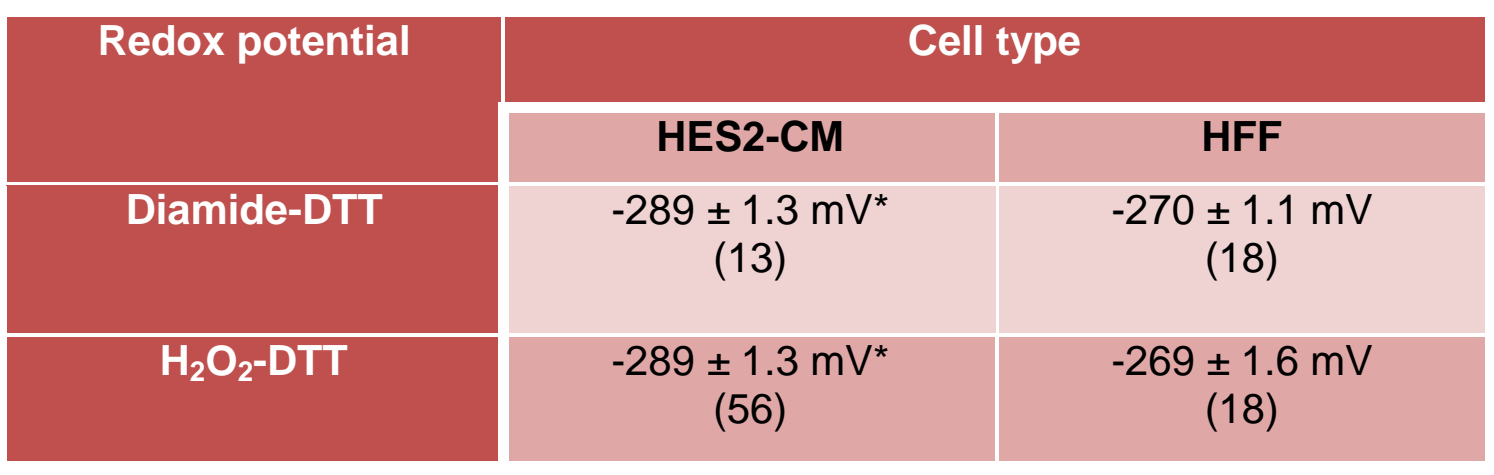

Table 10: $\mathrm{E}_{\mathrm{GSH}}$ of the glutathione sensor in HFFs and HES2-CMs. Number of analyzed cells is shown inside the parentheses. ${ }^{*} p<0.05$ HES2-CM vs. HFF by unpaired, two-tailed Student's t-test.

\subsubsection{Glutathione redox changes upon cumulative redox challenge}

In addition to the effects of bolus administration of $\mathrm{H}_{2} \mathrm{O}_{2}$ and DTT, responses to cumulative additions were analysed in HFFs (Figure 29). These analyses suggest a good dynamic range of the Grx1-roGFP2 sensor with the possibility to sense oxidation by $10-1,000 \mu \mathrm{mol} / \mathrm{L} \mathrm{H}_{2} \mathrm{O}_{2}$ and reduction by $0.01-1 \mathrm{mmol} / \mathrm{L}$ DTT.

A)

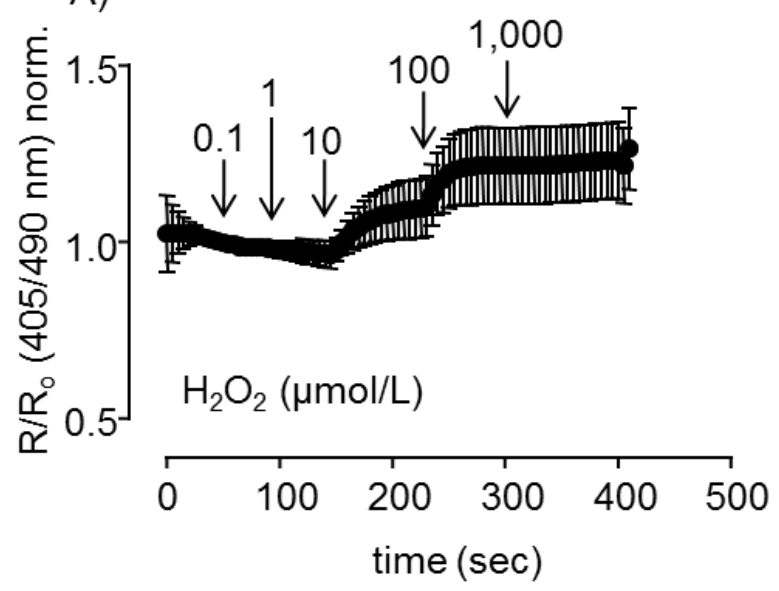

B)

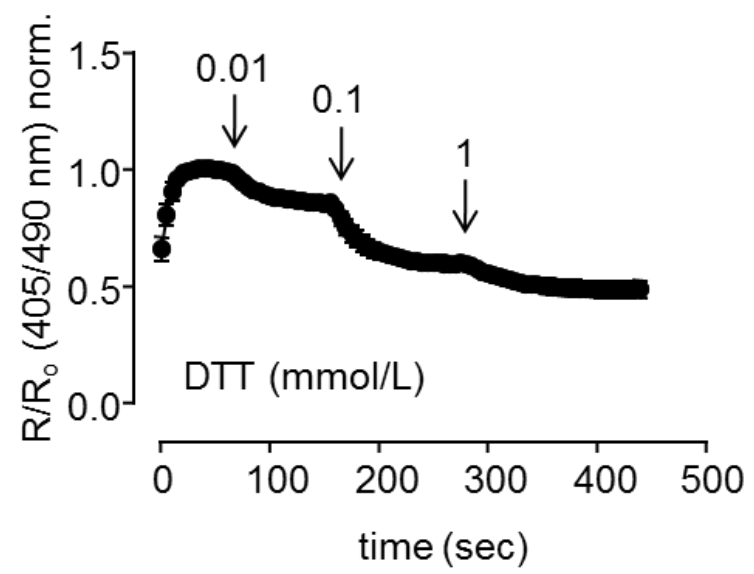

Figure 29: Assessment of the dynamic range of the Grx1-roGFP2 sensor. Stimulation with (A) $\mathrm{H}_{2} \mathrm{O}_{2}$ (18 cells/group) and (B) DTT (8 cells/group). 


\subsection{Grx1-roGFP2 responses to angiotensin II and inotropes}

Angiotensin II has been demonstrated to increase ROS/roGFP2 signal in murine heart preparations (Swain et al. 2016). In HFF and HES2-CM there was a distinct response to Angiotensin II with at all a mild reductive effect in HFFs at $100 \mathrm{nmol} / \mathrm{L}$ (Figure 30), but a strong oxidative effect in HES2-CM already at low $(0.1 \mathrm{nmol} / \mathrm{L})$ angiotensin II concentrations (Figure 31); notably, there appeared to be a concentration dependent decline of this observed oxidative response.
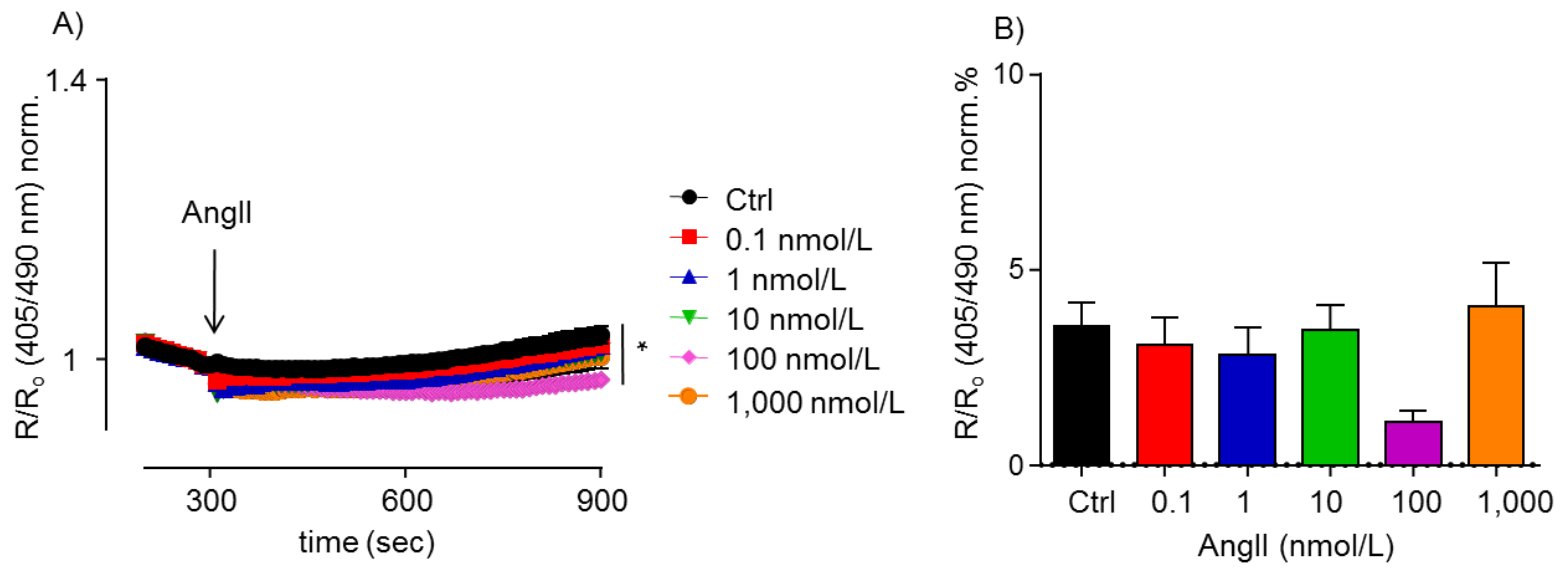

Figure 30: Visualization of glutathione redox states under angiotensin II stimulation in HFF. (A) Changes in the ratiometric fluorescence signal of the Grx1roGFP2 in HFF in response to angiotensin II (Angll) at the indicated concentrations as a function of time ( $R_{0}=405 / 490 \mathrm{~nm}$ excited signals at baseline); angiotensin II was added at $300 \mathrm{sec}$ of stable baseline recordings and (B) Maximal ratiometric signal increase at the indicated angiotensin II (Angll) concentrations. 42-68 cells/group; ${ }^{*} \mathrm{p}<0.05$ vs. Ctrl by two-way (A) and one-way (B) ANOVA followed by Dunnett's post hoc test. 
A)

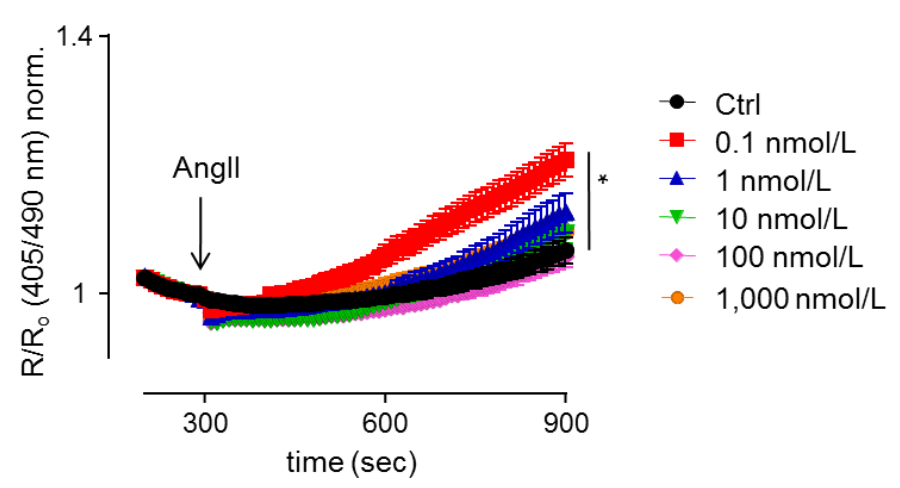

B)

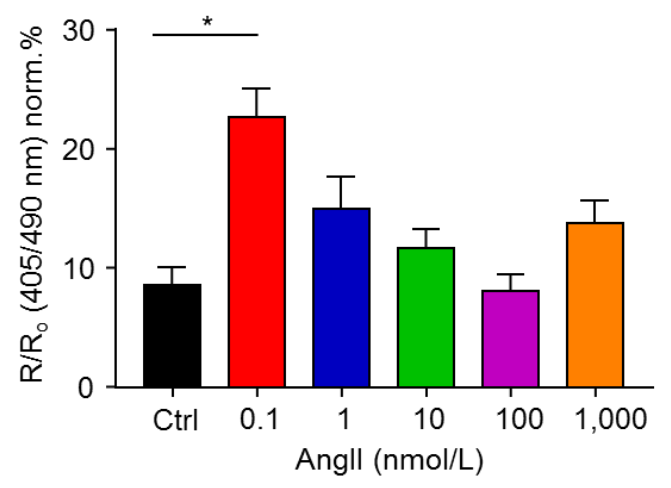

Figure 31: Visualization of glutathione redox states under angiotensin II stimulation in HES2-CM. (A) Changes in the ratiometric fluorescence signal of the Grx1-roGFP2 in HES2-CM in response to angiotensin II (Angll) at the indicated concentrations as a function of time $\left(R_{0}=405 / 490 \mathrm{~nm}\right.$ excited signals at baseline); angiotensin was added at $300 \mathrm{sec}$ of stable baseline recordings and (B) Maximal ratiometric signal increase at the indicated angiotensin II (Angll) concentrations. 3171 cells/group; ${ }^{*} p<0.05$ vs. Ctrl by two-way (A) and one-way (B) ANOVA followed by Dunnett's post hoc test.

Next, the cells were exposed to increasing concentrations of the calcium-sensitizer levosimendan (LEVO; $0.01-10 \mu \mathrm{mol} / \mathrm{L}$ ). Similarly as observed for angiotensin II, there was an effect in both HES2-CM (Figure 32) and HFF (Figure 33). The apparent increase in oxidation in the Ctrl group appeared to be an effect of the solvent (DMSO) and may have to be considered when interpreting the data. In all the experiments with levosimendan (LEVO) and omecamtiv mecarbil (OME), the Ctrl group includes $1 \%$ DMSO, whereas the sample dilutions $(0.01-10 \mu \mathrm{mol} / \mathrm{L})$ contain less DMSO $(0.01 \%$ or less DMSO). 


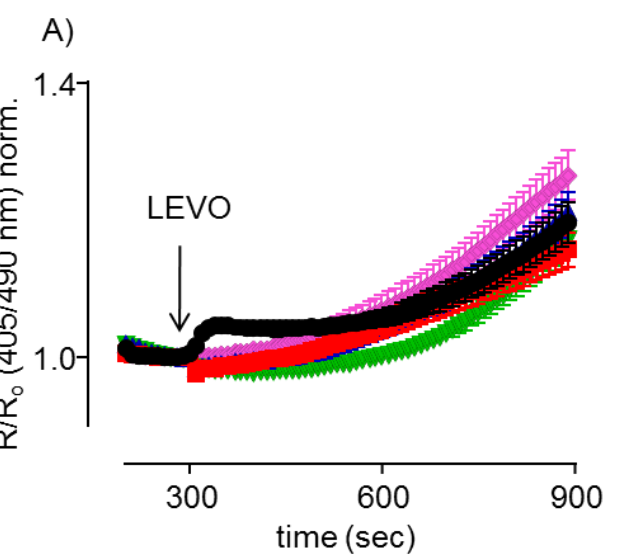

B)

- Ctrl with DMSO

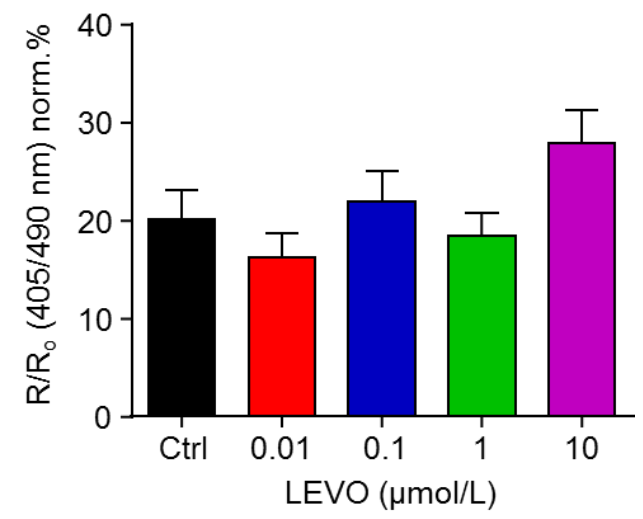

Figure 32: Visualization of glutathione redox states under levosimendan treatment in HFF. (A) Changes in the ratiometric fluorescence signal of the Grx1roGFP2 in HFF in response to levosimendan (LEVO) at the indicated concentrations as a function of time $\left(R_{0}=405 / 490 \mathrm{~nm}\right.$ excited signals at baseline); levosimendan was added at $300 \mathrm{sec}$ of stable baseline recordings and (B) Maximal ratiometric signal increase at the indicated levosimendan (LEVO) concentrations (17-41 cells/group).

Surprisingly, the sample dilutions of levosimendan did not show any significant difference on oxidizing effect when compared to the Ctrl group in HFFs (Figure 32). However, Ctrl group in HES2-CMs treated with levosimendan showed a more pronounced oxidizing effect in comparison to the sample dilutions (Figure 33). 
A)

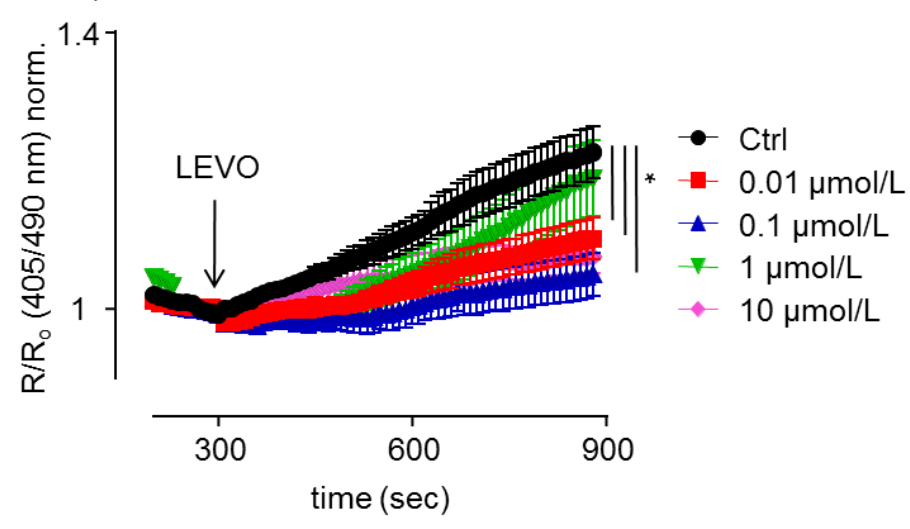

B)

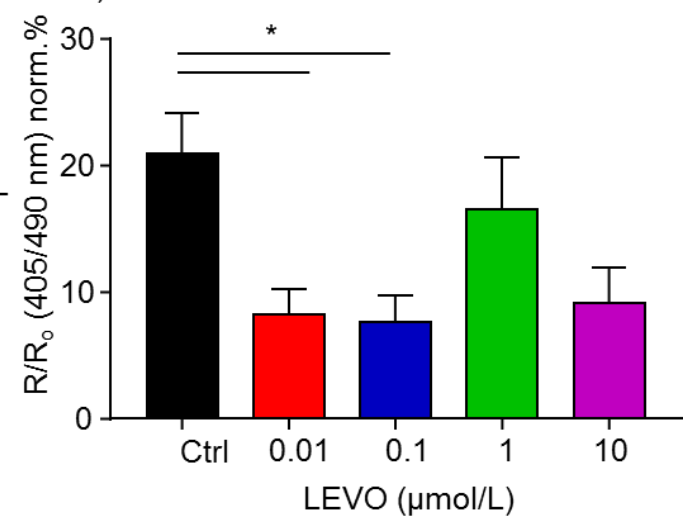

Figure 33: Visualization of glutathione redox states under levosimendan treatment in HES2-CM. (A) Changes in the ratiometric fluorescence signal of the Grx1-roGFP2 in HES2-CM in response to levosimendan (LEVO) at the indicated concentrations as a function of time $\left(R_{0}=405 / 490 \mathrm{~nm}\right.$ excited signals at baseline); levosimendan was added at $300 \mathrm{sec}$ of stable baseline recordings and (B) Maximal ratiometric signal increase at the indicated levosimendan (LEVO) concentrations. 921 cells/group; ${ }^{*}<<0.05$ vs. Ctrl by two-way (A) and one-way (B) ANOVA followed by Dunnett's post hoc test.

Finally, the cells were exposed to omecamtiv mercabil (OME; $0.01-10 \mu \mathrm{mol} / \mathrm{L}$ ), a cardiac myosin activator. In contrast to Angll and LEVO, a reduced Grx1-roGFP2 signal was observed under OME stimulation only in HES2-CM without a clear concentration dependency (Figure 35). Similar as for the LEVO experiments, the oxidative effects of DMSO have to be considered, which appeared more pronounced in HES2-CM than HFF. 

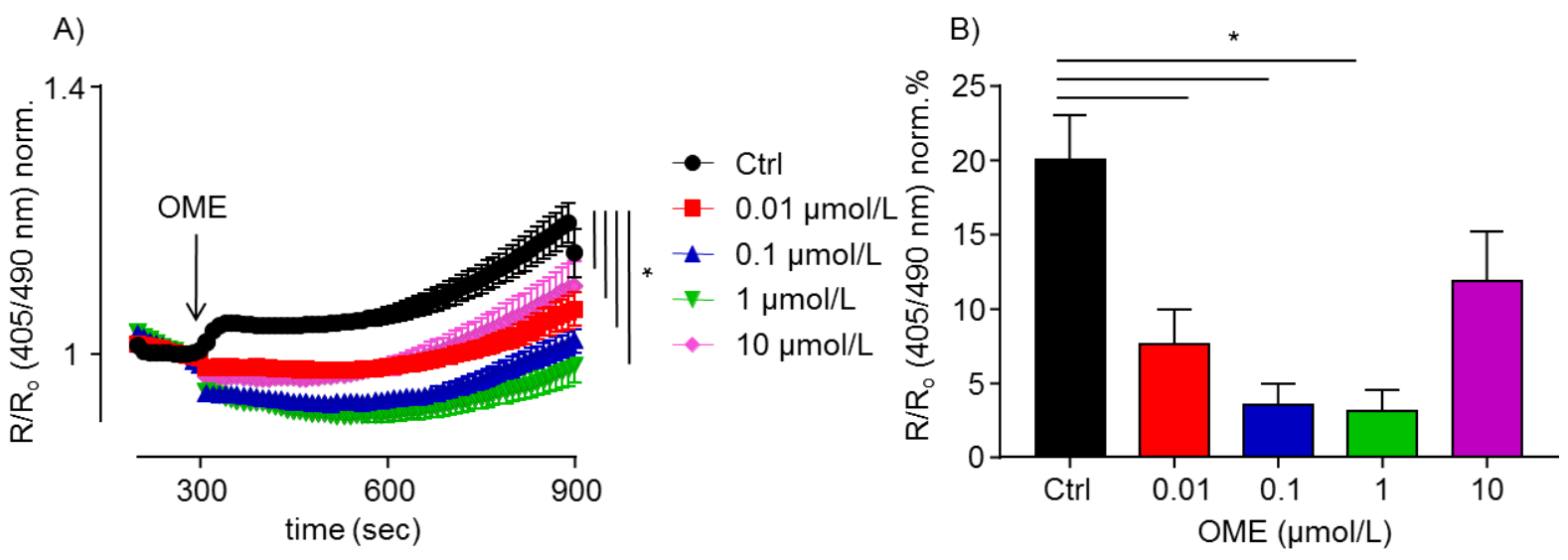

Figure 34: Visualization of glutathione redox states under omecamtiv mecarbil treatment in HFF. (A) Changes in the ratiometric fluorescence signal of the Grx1roGFP2 in HFF in response to omecamtiv mecarbil (OME) at the indicated concentrations as a function of time $\left(R_{0}=405 / 490 \mathrm{~nm}\right.$ excited signals at baseline); omecamtiv mecarbil was added at $300 \mathrm{sec}$ of stable baseline recordings and (B) Maximal ratiometric signal increase at the indicated omecamtiv mecarbil (OME) concentrations. $13-22$ cells/group; ${ }^{*} p<0.05$ vs Ctrl by two-way (A) and one-way (B) ANOVA followed by Dunnett's post hoc test.

A)

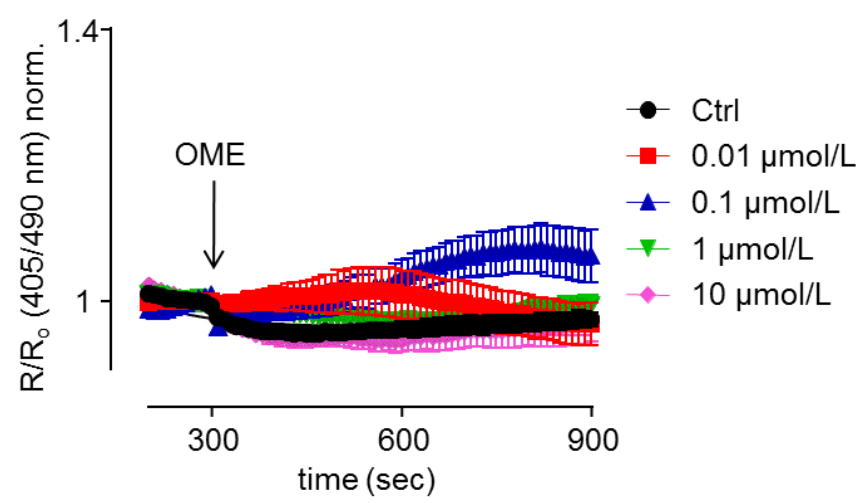

B)

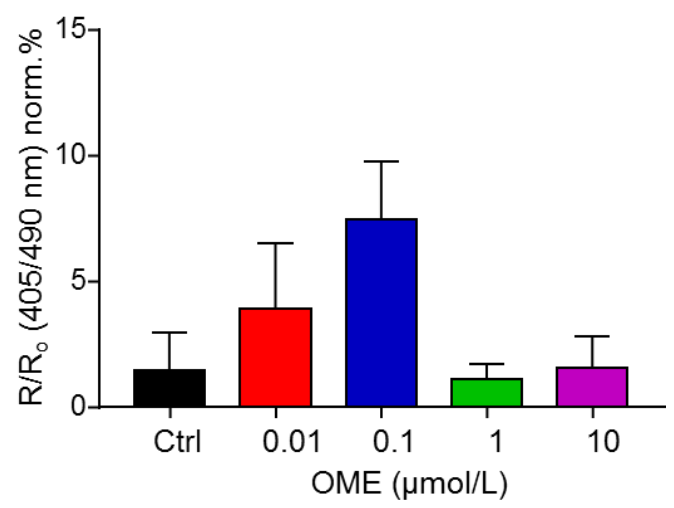

Figure 35: Visualization of glutathione redox states under omecamtiv mecarbil treatment in HES2-CM. (A) Changes in the ratiometric fluorescence signal of the Grx1-roGFP2 in HES2-CM in response to omecamtiv mecarbil (OME) at the indicated concentrations as a function of time $\left(R_{0}=405 / 490 \mathrm{~nm}\right.$ excited signals at baseline); omecamtiv mecarbil was added at $300 \mathrm{sec}$ of stable baseline recordings and (B) Maximal ratiometric signal increase at the indicated omecamtiv mecarbil (OME) concentrations (4-7 cells/group). 


\subsection{Detection of peroxiredoxin oxidation}

Exposure of cells to oxidative stress (e.g., by exposure to $\mathrm{H}_{2} \mathrm{O}_{2}$ ) is anticipated to result in the oxidation of cysteines (Cys-SH) in a wide array of proteins, including peroxiredoxin (Prx). Cysteines in Prx are reversibly oxidized to sulfenic acid groups ($\mathrm{SOH})$ and irreversibly to sulfinic $\left(-\mathrm{SO}_{2} \mathrm{H}\right)$ or sulfonic acid groups $\left(-\mathrm{SO}_{3} \mathrm{H}\right)$. Immunoblot detection of periodoxin- $\mathrm{SO}_{3}$ (Table 8) confirmed that $\mathrm{H}_{2} \mathrm{O}_{2}$ at the above as oxidatively effective identified concentrations (100 - 1,000 $\mu \mathrm{mol} / \mathrm{L})$ in HFF and HES2-CM indeed resulted in the anticipated oxidation of periodoxin (Figure 36). Interestingly, Grx1roGFP2 sensor appeared 10-fold more sensitive as to the detection of oxidation with clearly enhanced signals already at $10 \mu \mathrm{mol} / \mathrm{L}$ (Figure 20 and 21).

A)

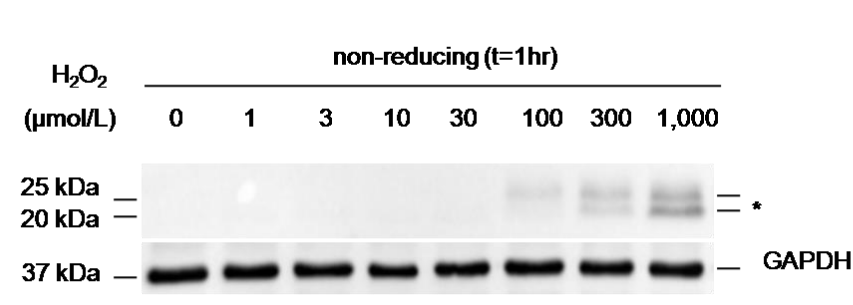

C)

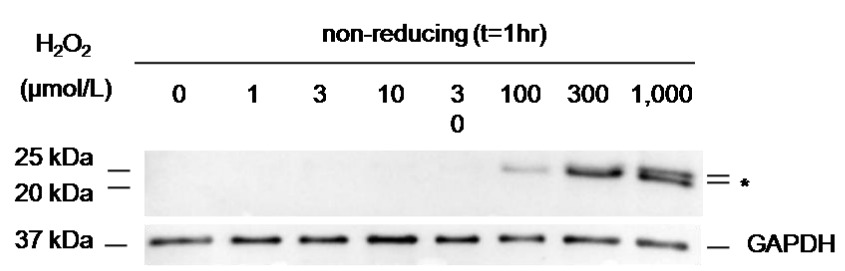

${ }^{*}$ Peroxiredoxin $-\mathrm{SO}_{2}-\mathrm{SO}_{3}{ }^{-}$, expected size $22-25 \mathrm{kDa}$
B)

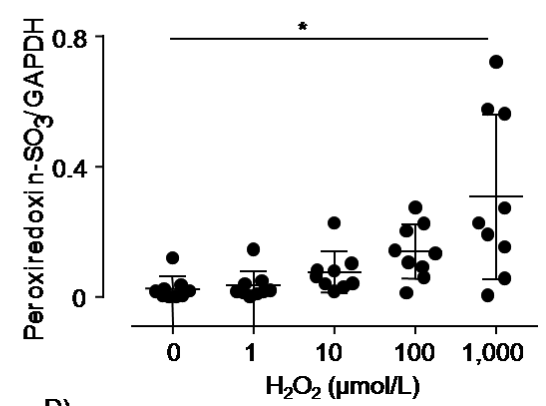

D)

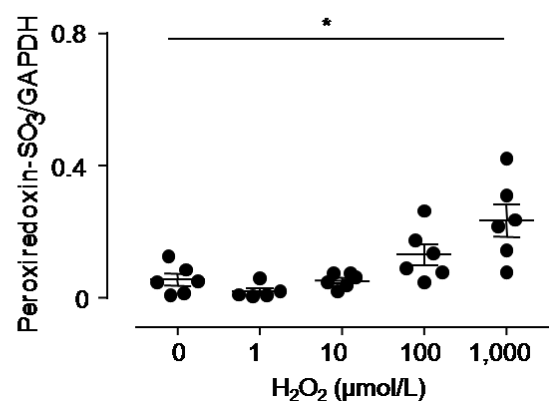

Figure 36: Confirmation of peroxiredoxin oxidation in HFFs and HES2-CMs upon $\mathrm{H}_{2} \mathrm{O}_{2}$ exposure. Protein lysates were prepared $1 \mathrm{hr}$ after exposure to the indicated $\mathrm{H}_{2} \mathrm{O}_{2}$ concentration. Protein oxidation was stabilized with maleimide. Representative Immunoblots and analyses of all obtained data $\left(\mathrm{Prx}-\mathrm{SO}_{3}\right.$ signals were corrected to GAPDH signals). (A-B) data from HFF cultures ( $n=9 /$ group); (C-D) data from HES2-CM cultures ( $n=5-6 /$ group). ${ }^{*} p<0.05$ vs. Ctrl by one-way ANOVA followed by Dunnett's post hoc test. 


\subsection{Redox sensing in engineered heart muscle}

To assess whether changes of the glutathione redox state in HFFs and HES2-CMs can be determined in a multicellular human tissue context, EHM (Tiburcy et al. 2017) were constructed from distinctly labelled (Grx1-roGFP2) mixtures of HFF and HES2CM (Figure 37).

A)

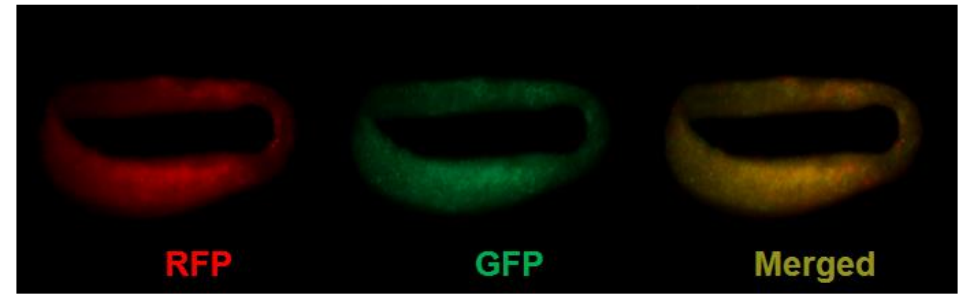

B)

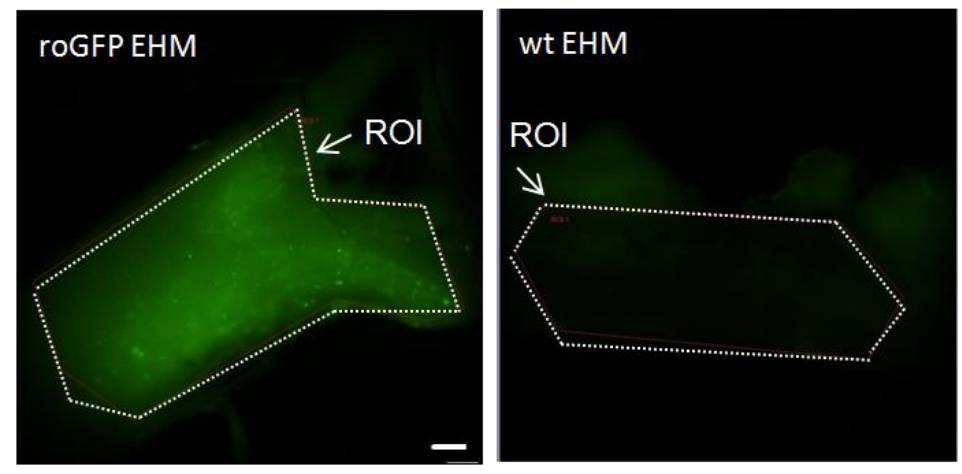

Figure 37: Expression and monitoring of the glutathione redox sensor in EHM.

(A) EHM with Grx1-roGFP2 glutathione redox sensor expressed in roGFP HFF (B) Zoom in on a ROI for roGFP2 detection in EHM (left); control EHM without the expression of Grx1-roGFP2 (right). Scale bar: $200 \mu \mathrm{m}$.

\subsubsection{Glutathione redox measurements in EHM}

EHM with either HFF or HES2-CM expressing Grx1-roGFP2 were exposed to $\mathrm{H}_{2} \mathrm{O}_{2}$ and DTT at maximally effective concentrations (1 mmol/L; Figure 38). These experiments confirmed the utility of the Grx1-roGFP2 sensor in multicellular human tissue preparations and in addition demonstrated the concept of cell type specific redox sensing in HFF (Figure 38A) and HES2-CM (Figure 38B). Oxidative challenge with $\mathrm{H}_{2} \mathrm{O}_{2}$ induced a similar effect on HFF and HES2-CM in EHM, whereas HES2- 
CM appeared to respond with a stronger decrease in sensor signal upon DTT challenge.

A)

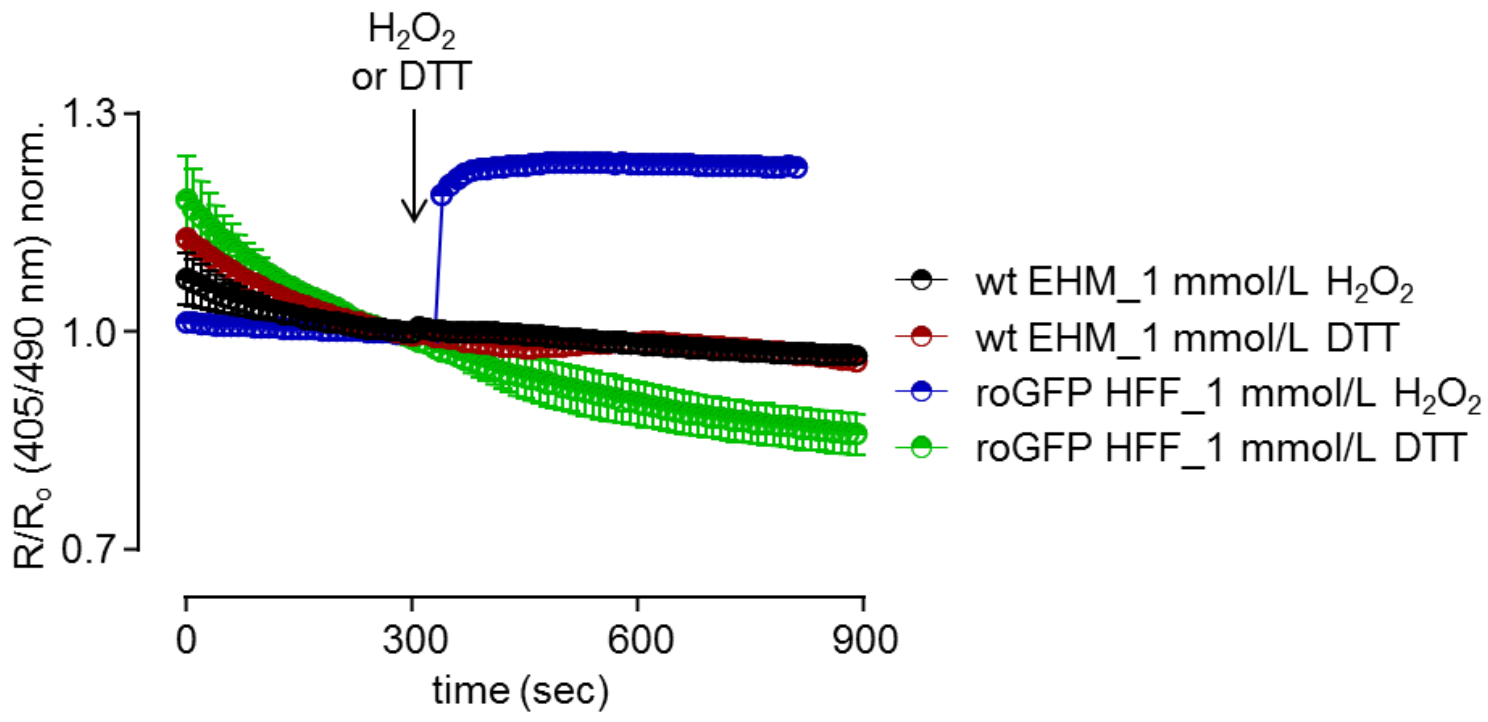

B)

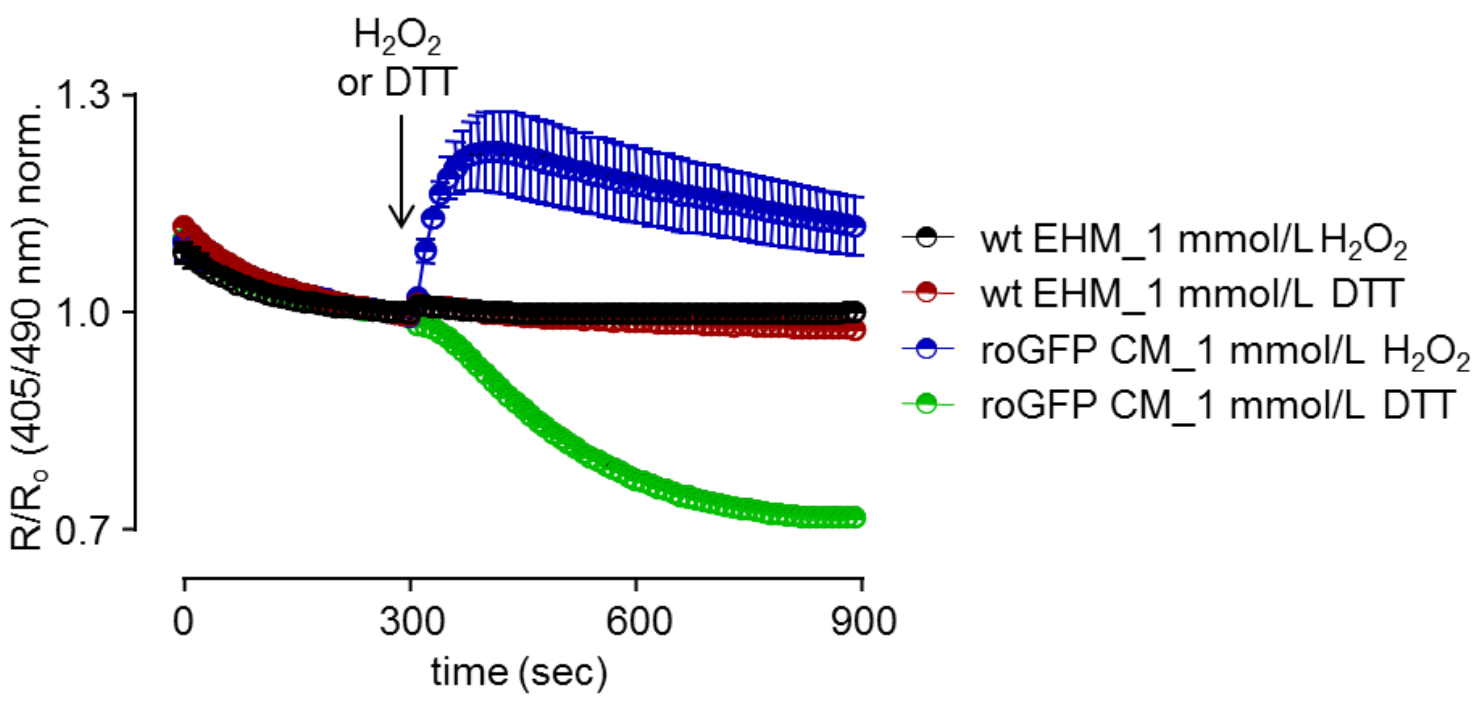

Figure 38: Cell type specific visualization of redox alteration in engineered human myocardium. Changes in the ratiometric fluorescence signal of the Grx1roGFP2 in (A) HFF (1-2 EHM/group) and (B) HES2-CM (2-4 EHM/group) in response to $\mathrm{H}_{2} \mathrm{O}_{2}(1 \mathrm{mmol} / \mathrm{L})$ and DTT $(1 \mathrm{mmol} / \mathrm{L})$ as a function of time $\left(\mathrm{R}_{0}=405 / 490 \mathrm{~nm}\right.$ excited signals at baseline); $\mathrm{H}_{2} \mathrm{O}_{2}$ or DTT were added as indicated at $300 \mathrm{sec}$ of stable baseline recordings. 


\subsubsection{Contraction force measurement}

EHM were developed under biomimetic conditions to obtain functional properties of bona fide myocardium, which can be measured under defined isometric conditions (Figure 39) (Tiburcy et al. 2017). Inotropic properties of EHM were analysed under exposure to increasing extracellular calcium concentrations. This confirmed similar contractility independent of Grx1-roGFP2 expression and thus ruled out sensor toxicity.

A)

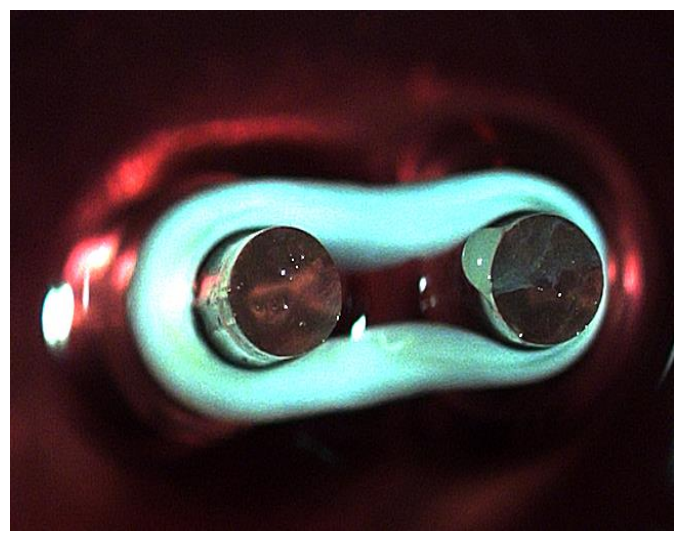

C)

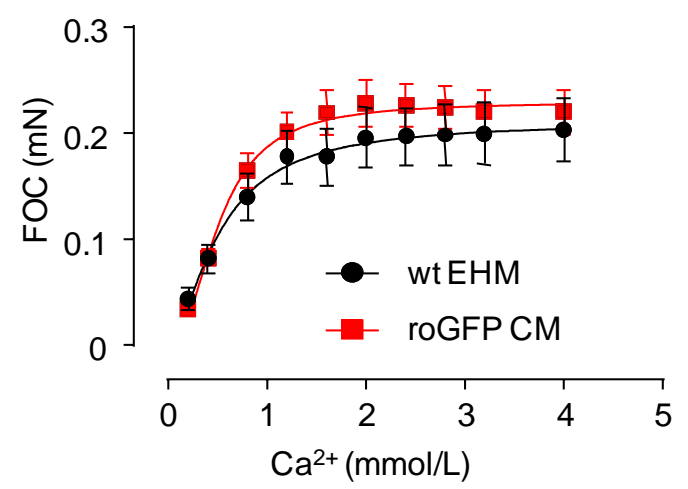

B)

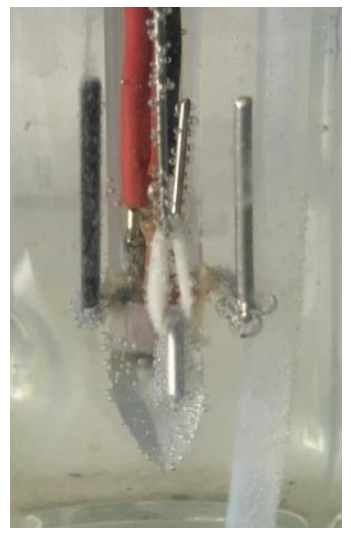

D)

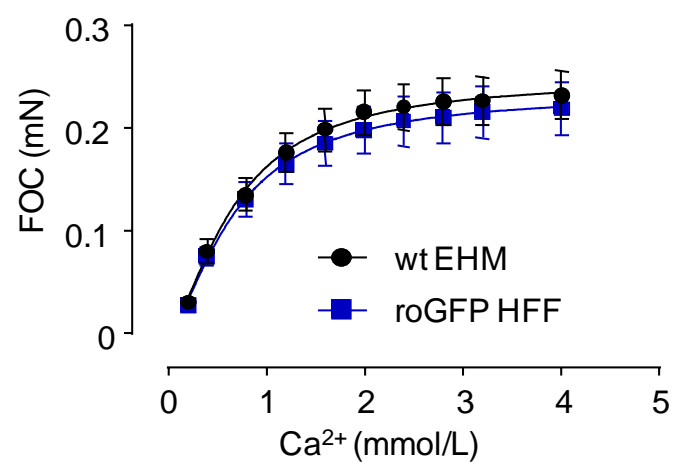

Figure 39: Assessment of potential Grx1-roGFP2 toxicity in EHM. (A) EHM during culture on flexible silicone poles to facilitate auxotonic contractions. (B) EHM suspended in an organ bath filled with Tyrode's solution at $37^{\circ} \mathrm{C}$ for isometric force measurements under electrical field stimulation $(1.5 \mathrm{~Hz})$. Force of contraction (FOC) under increasing extracellular calcium concentrations as indicated in EHM comprising (C) Grx1-roGFP2 HES2-CM (n= 17-33/group) or (D) Grx1-roGFP2 HFF ( $\mathrm{n}=36$ 42/group). 
Finally, concentration response curves for $\mathrm{H}_{2} \mathrm{O}_{2}$ and DTT were analysed to determine whether oxidative or reductive challenges would affect human EHM contractility. This set of experiments demonstrated surprisingly little acute effects at a wide range of concentrations tested (Figure 40). However at highest oxidative $\left(\mathrm{H}_{2} \mathrm{O}_{2}: 1 \mathrm{mmol} / \mathrm{L}\right.$ ) and reductive challenge (DTT: $3 \mathrm{mmol} / \mathrm{L}$ ) contractile function was reduced and increased, respectively.

A)

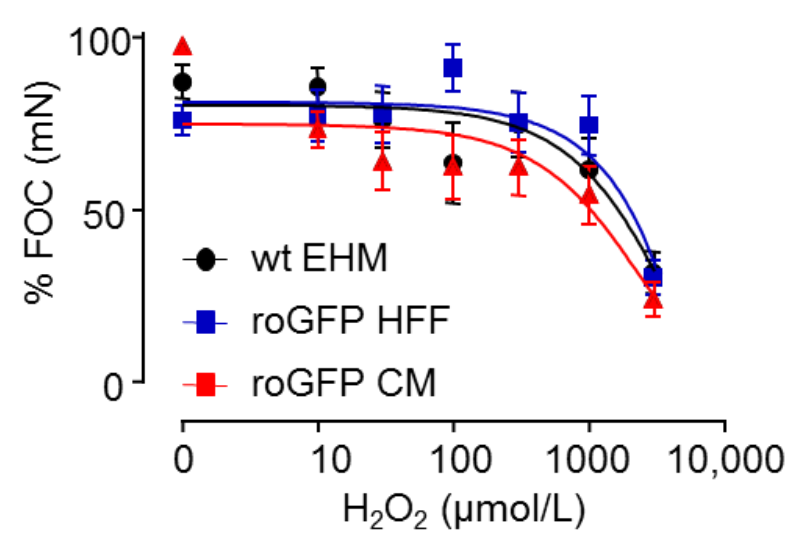

B)

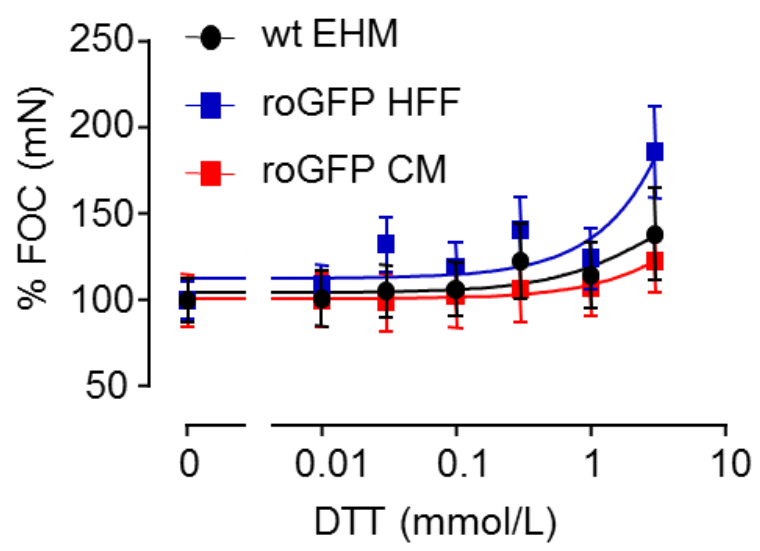

Figure 40: Functional consequences of oxidation and reduction in EHM contractility. EHM were exposed to increasing concentrations of $\mathrm{H}_{2} \mathrm{O}_{2}(\mathbf{A} ; n=9$ 15/group) or DTT (B; $n=6-19 /$ group) as indicated. FOC was assessed isometrically under electrical field stimulation $(1.5 \mathrm{~Hz})$ at $\mathrm{EC}_{50}$ calcium concentrations (individually determined prior to exposure to $\mathrm{H}_{2} \mathrm{O}_{2}$ or DTT). EHM from all tested groups demonstrated similar responses, confirming that lentiviral transduction with Grx1roGFP2 did not impair HFF or HES2-CM function. 


\section{Discussion}

Redox signaling affects the heart both physiologically (e.g., cell growth, differentiation, excitation-contraction coupling) and pathologically (e.g., fibrosis, cardiac remodeling, hypertrophy) (Burgoyne et al. 2012, Santos et al. 2011). Cells are equipped with redox-stress defense mechanisms that include endogenous antioxidants (e.g., glutathione, glutathione peroxidase, thioredoxin, peroxiredoxin) (Hafstad et al. 2013, Santos et al. 2011). Therapeutic application of compounds with antioxidant activity has been proposed as an approach to protect the heart, with however so far limited success. A key challenge to the field is to determine the mechanisms underlying the fine-tuning of the redox status in cells and its subcellular compartments. The application of redox assays/sensors promises for detailed insight into qualitative and quantitative changes of the redox state of the cell. Until recently, most studies have utilized a variety of redox sensitive dyes, such as dichlorodihydrofluorescein diacetate, amplex red, and dihydrorhodamine (Forkink et al. 2010, Griendling et al. 2016, Meyer and Dick 2010) to monitor both ROS in and redox states of cells. The recent advent of genetically encoded redox sensors promises to advance the field markedly because of the possibility to target sensors to subcellular compartments (Swain et al. 2016) or cell types, as demonstrated in this thesis.

The following main observation could be made:

1) Human fibroblasts and cardiomyocytes can be stably transduced with the cytosolic glutathione redox sensor Grx1-roGFP2 without signs for transgene toxicity.

2) Cardiomyocytes are more sensitive to oxidation and less reactive to reduction compared to fibroblasts. 
3) This may at least in part be explained by the more reduced glutathione redox potential in cardiomyocytes compared to fibroblasts.

4) Fibroblasts as compared to cardiomyocytes displayed a wider range in redox state alterations.

5) Fibroblasts show faster responses to oxidative and reductive challenges compared to cardiomyocytes.

6) Assessment of redox alterations under pharmacological stimulation was confounded by oxidizing effects of DMSO.

7) Glutathione redox sensing can be performed in a cell type specific manner in EHM and thus may allow dissecting the "redox-interplay" between different cell types in a tissue context.

Fibroblast from human foreskin and cardiomyocytes derived from the human embryonic stem cell line HES2 were utilized as surrogates for the most abundant cell types of the heart. The suitability of these cells to model human heart function was recently demonstrated (Tiburcy et al. 2017).

\subsection{Tools for redox quantification in living cells}

Monitoring of redox alteration in cells and tissue requires tools for the robust and quantitative reporting of the dynamic changes in cellular redox states. The most promising tools to monitor cellular redox changes dynamically, in real time and compartment specific appear to be biological sensors genetically integrated into cells of interest (Lukyanov and Belousov 2014, Meyer and Dick 2010). In this study, we utilized the genetically encoded glutathione redox sensor Grx1-roGFP2, which detects redox changes of the GSH:GSSG couple in a quantitative manner in real time. Modifications of the Grx1-roGFP2 sensor have been shown to also allow for subcellular targeting of the redox sensor (Swain et al. 2016). This study identified differences in the cytosol and mitochondria of murine cardiomyocytes. In this thesis, targeting of the most abundant cell types in the human heart was demonstrated by 
transducing the cytosolic Grx1-roGFP2 sensor into cardiomyocytes and fibroblasts. Human embryonic stem (HES) cell-derived cardiomyocytes and human foreskin fibroblasts were chosen because of their accessibility and the finding that these cell types can be reconstituted to myocardium with structural, molecular, and functional properties of the postnatal human heart (Tiburcy et al. 2017). In fact, human foreskin and adult heart derived fibroblasts are similarly supportive to heart muscle reconstitution in vitro (unpublished data). Lentiviral transduction was chosen in a first attempt to validate the concept of Grx1-roGFP2 expression in human cardiomyocytes and fibroblasts cells. The need for repeated transduction especially in HES-derived cells makes this a tedious approach, which will have to be replaced by the stable introduction of the reporters by homologous recombination. In addition, silencing and incomplete labelling of the cells of interest is an issue that can be circumvented with for example TALEN or CRISPR mediated integration of target sequences in defined genomic loci such as the AAVS1 site (Mussolino et al. 2014). Despite these caveats it was possible to establish proof-of-concept for glutathione redox potential sensing in human heart cells. Validation experiments with oxidizing $\left(\mathrm{H}_{2} \mathrm{O}_{2}, \mathrm{DA}\right)$ and reducing (DTT) compounds confirmed the principle utility of the Grx1-roGFP2 sensor in human cardiomyocytes and fibroblasts. Some of the observed variability may be contributed to variable transduction and activity of the Grx1-roGFP2 sensor.

Another robust way to analyze cellular oxidation is by the investigation of characteristic protein modifications under oxidative stimulation. For example, effective oxidation of peroxiredoxin by $\mathrm{H}_{2} \mathrm{O}_{2}$ should lead to its sulfonylation (- $\mathrm{SO}_{3}$ ) (Sobotta et al. 2013), which can be detected by $\mathrm{Prx}_{-} \mathrm{SO}_{3}$ specific antibodies. Immunoblots confirmed that $\mathrm{H}_{2} \mathrm{O}_{2}$ at the chosen concentration range would indeed result in oxidation of $\mathrm{Prx}$ with an apparent $\mathrm{H}_{2} \mathrm{O}_{2}$ concentration dependent effect at 10 - $1,000 \mu \mathrm{mol} / \mathrm{L} \mathrm{H}_{2} \mathrm{O}_{2}$.

\subsection{Cell type specific glutathione redox responses}

Both HFFs and HES2-CMs demonstrated a concentration dependent oxidizing and reducing trajectory with distinct sensitivities $\left(\mathrm{EC}_{50}\right)$, response kinetics ( $\left.\mathrm{t}_{50}\right)$, and $\mathrm{E}_{\mathrm{GSH}}$ (Table 11). 


\begin{tabular}{|c|c|c|c|}
\hline & Parameter & HES2-CM (n) & HFF (n) \\
\hline \multirow[b]{3}{*}{$\mathrm{H}_{2} \mathrm{O}_{2}$} & $\mathrm{EC}_{50}$ & $2.6 \pm 2.2 \mu \mathrm{mol} / \mathrm{L}(46-70)$ & $10.5 \pm 1.2 \mu \mathrm{mol} / \mathrm{L}(19-43)$ \\
\hline & $R / R_{0}(\max )$ & $+56 \pm 4 \%(57)$ & $+78 \pm 7 \%(41)$ \\
\hline & $t_{50}$ & $\begin{array}{l}10 \mu \mathrm{mol} / \mathrm{L}=26 \pm 1 \mathrm{~ms}(45) \\
100 \mu \mathrm{mol} / \mathrm{L}=26 \pm 1 \mathrm{~ms}(39) \\
1,000 \mu \mathrm{mol} / \mathrm{L}=22 \pm 2 \mathrm{~ms}(39)\end{array}$ & $\begin{array}{l}10 \mu \mathrm{mol} / \mathrm{L}=30 \pm 3 \mathrm{~ms}(27) \\
100 \mu \mathrm{mol} / \mathrm{L}=6 \pm 0.3 \mathrm{~ms}(41) \\
1,000 \mu \mathrm{mol} / \mathrm{L}=5 \pm 0.3 \mathrm{~ms}(41)\end{array}$ \\
\hline \multirow[b]{3}{*}{ DA } & $\mathrm{EC}_{50}$ & $16.1 \pm 1.4 \mu \mathrm{mol} / \mathrm{L}(7-19)$ & $40.7 \pm 1.2 \mu \mathrm{mol} / \mathrm{L}(42-60)$ \\
\hline & $R / R_{0}(\max )$ & $+57 \pm 4 \%(14)$ & $+153 \pm 4 \%(48)$ \\
\hline & $t_{50}$ & $\begin{array}{l}10 \mu \mathrm{mol} / \mathrm{L}=49 \pm 4 \mathrm{~ms}(55) \\
100 \mu \mathrm{mol} / \mathrm{L}=36 \pm 2 \mathrm{~ms}(39) \\
1,000 \mu \mathrm{mol} / \mathrm{L}=9 \pm 1 \mathrm{~ms}(23)\end{array}$ & $\begin{array}{l}10 \mu \mathrm{mol} / \mathrm{L}=27 \pm 1 \mathrm{~ms}(7) \\
100 \mu \mathrm{mol} / \mathrm{L}=28 \pm 3 \mathrm{~ms}(13) \\
1,000 \mu \mathrm{mol} / \mathrm{L}=25 \pm 1 \mathrm{~ms}(15)\end{array}$ \\
\hline \multirow[b]{3}{*}{ DTT } & $\mathrm{EC}_{50}$ & $0.2 \pm 0.1 \mathrm{mmol} / \mathrm{L}(46-85)$ & $0.03 \pm 0.01 \mathrm{mmol} / \mathrm{L}(28-45)$ \\
\hline & $R / R_{o}(\max )$ & $-22 \pm 1 \%(58)$ & $-64 \pm 2 \%(28)$ \\
\hline & $t_{50}$ & $\begin{array}{l}0.01 \mathrm{mmol} / \mathrm{L}=131 \pm 16 \mathrm{~ms}(62) \\
0.1 \mathrm{mmol} / \mathrm{L}=115 \pm 11 \mathrm{~ms}(40) \\
1 \mathrm{mmol} / \mathrm{L}=83 \pm 3 \mathrm{~ms}(58)\end{array}$ & $\begin{array}{l}0.01 \mathrm{mmol} / \mathrm{L}=42 \pm 4 \mathrm{~ms}(21) \\
0.1 \mathrm{mmol} / \mathrm{L}=31 \pm 1 \mathrm{~ms}(37) \\
1 \mathrm{mmol} / \mathrm{L}=28 \pm 1 \mathrm{~ms}(28)\end{array}$ \\
\hline $\mathrm{E}_{\mathrm{GSH}}$ & $\mathrm{H}_{2} \mathrm{O}_{2}$-DTT & $-289 \pm 1 m V(56)$ & $-269 \pm 2 m V(18)$ \\
\hline
\end{tabular}

Table 11: Summary of Grx1-roGFP2 sensor activites in HES2-CM and HFF. $\mathrm{n}=$ numbers of cells analysed.

The more reduced $\mathrm{E}_{\mathrm{GSH}}$ in cardiomyocytes was a particularly interesting observation and has to be considered when interpreting the observed differences in the responses to oxidative and reductive challenges. The response range to oxidative and reductive stimulation with $\mathrm{H}_{2} \mathrm{O}_{2}$ and DTT, respectively, was for both cell types 10 - 1,000 $\mu \mathrm{mol} / \mathrm{L}$ and $0.01-1 \mathrm{mmol} / \mathrm{L}$. HES2-CM showed a markedly higher sensitivity to oxidation (refer to $\mathrm{EC}_{50}$ in Table 11) with at the same time a clearly smaller dynamic range in redox alterations (refer to $R / R_{0}$ in Table 11); cardiomyocytes 
demonstrated a $\Delta R / R_{0}$ differences of $\sim 80 \%$ vs. $\sim 140 \%$ in fibroblasts (absolute $\%$ change). Another interesting observation was the apparent roGFP-oxidation reserve in fibroblasts, which was evidenced by the lower $\mathrm{R} / \mathrm{R}_{0}$ signal change under maximal $\mathrm{H}_{2} \mathrm{O}_{2}(\sim 80 \%)$ vs. DA ( 150\%).

Fibroblasts appear to exhibit a particular high expression in NOX4 (RNA-sequencing data in (Tiburcy et al. 2017)). NOX4 is constitutively active and $\mathrm{H}_{2} \mathrm{O}_{2}$ producing (Kuroda et al. 2010) and moreover directly activated by $\mathrm{H}_{2} \mathrm{O}_{2}$ mediated oxidation (Colston et al. 2005). This suggests a more extensive oxidant load in fibroblasts and thus may on the one hand explain the difference in $\mathrm{E}_{\mathrm{GSH}}$ (Table 11) and the wider range in particular of oxidative changes of the redox state. The difference in oxidation/reduction kinetics ( $\left.t_{50}\right)$ was a surprising observation and may argue for differences in cellular uptake or membrane permeability of $\mathrm{H}_{2} \mathrm{O}_{2}$. There is a steep gradient of $\mathrm{H}_{2} \mathrm{O}_{2}$ between extra- and intracellular compartments with a 100 -fold higher concentration outside vs. inside the cell, in addition there are differences in $\mathrm{H}_{2} \mathrm{O}_{2}$ in the different subcellular compartments (Sies 2017). The difference in $t_{50}$ reported in this thesis requires more detailed investigations of differences in transmembranous $\mathrm{H}_{2} \mathrm{O}_{2}$ gradients as well as subcellular targets in cardiomyocytes and fibroblasts. The recent demonstration of cytosol and mitochondria targeting of the Grx1-roGFP2 sensor is particularly interesting in this context (Swain et al. 2016).

\subsection{Cell type specific redox responses to angiotensin II and drugs}

Another aspect of this study was to investigate cell type redox responses upon stimulation with angiotensin II and inotropic drugs. From previous studies, stimulation with angiotensin II, the key effector of rennin angiotensin system, is known to be involved in ROS production pathways by stimulating membrane bound $N A D(P) H$ oxidase (Dikalov and Nazarewicz 2013, Griendling et al. 1994, Vazquez-Medina et al. 2013). In our experiments, HFFs did not exhibit obvious redox changes over the whole range of angiotensin II concentrations tested; surprisingly, a reductive effect was observed under $100 \mathrm{nmol} / \mathrm{L}$ angiotensin II. This is in contrast to the study conducted by Sano et al. and Swain et al. They demonstrated ROS production and roGFP2 oxidation in cardiac fibroblasts by angiotensin II stimulation (Sano et al. 
2001, Swain et al. 2016). HES2-CMs showed however some oxidative effects at a low angiotensin concentration $(0.1 \mathrm{nmol} / \mathrm{L})$. These results are surprising and somewhat inconsistent. Stable expression of Grx1-roGFP2 in every cell of interest with a similar expression level may help to further clarify these apparently discrepant findings. Collectively, these data suggest that assessments of oxidation and reduction would benefit from cell-type specific analyses.

In an attempt to study oxidative and reductive drug effects, levosimendan and omecamtiv mecarbil were applied to HES2-CM and HFF. These cardioactive inotropes are applied in acute heart failure with cardioprotective effects reported for levosimendan (via activation of the mitochondrial ATP-sensitive $\mathrm{K}^{+}$-channels) and no effect on myocardial oxygen consumption reported for omecamtiv mecarbil (Farmakis et al. 2016, Meijs et al. 2012). There was no obvious effect of LEVO and OME in cardiomyocyte or fibroblasts oxidation. However, these experiments appeared to be severely confounded oxidizing effects of the solvent DMSO. A refinement of the study protocol (avoidance of DMSO) is needed to ensure the assessment of drug specific effects and confirm the present findings of no effects of LEVO and OME on the redox state of cardiomyocytes and fibroblasts.

\subsection{Redox sensing in a heart muscle tissue context}

Up to this point, we discussed results and analysis that were performed in monolayer cardiomyocytes and fibroblasts. Cellular measurements provide information regarding compound effects and pathways involved in redox changes in specific cell cultures. However, investigations in a cardiac muscle model that mimic the microenvironment of the heart tissue are physiologically more relevant than monolayer cell culture. Furthermore, in a tissue context the interaction between cells plays a key role in the investigation of drug effects on redox homeostasis in a cardiac tissue. EHMs exhibit a higher degree of maturity as compared to monolayer cultures (resemble an embryonic phenotype); in fact, they reflect in many structural, molecular and functional parameters properties of the postnatal human heart (Tiburcy et al. 2017). By genetically modifying the two most abundant heart cell types and mixing them in a defined context, it became possible to define response to redox challenges in a heart muscle context. Importantly, we demonstrated that the biosensor 
responded in EHM to the bolus of $\mathrm{H}_{2} \mathrm{O}_{2}$ and DTT both optically and functionally. Stimulation of human EHMs with oxidizing and reducing agents confirmed that we were able to detect redox changes occurring within the tissue. Interestingly, contractile properties were deteriorated under maximal oxidation and appeared to be enhanced under maximal reduction with $\mathrm{H}_{2} \mathrm{O}_{2}$ and DTT, respectively. These results fit well with previous data showing that redox alterations affect signaling pathways (eg. Ca2+/calmodulin-dependent kinase II; CAMKII or cAMP-dependent protein kinase; PKA) important in contractility and could bring cardiomyocyte to death (Santos et al. 2016).

These studies will be extended in the future to study the interplay between the different cardiac cell species and their specific responses to environmental, biomechanical, and pharmacological stimuli. In addition, a link to signaling pathways will need to be established and assessments of acute vs chronic effects will need to be performed. A potential caveat is the thickness of engineered tissue $(\sim 1 \mathrm{~mm})$ and the intra-tissue distance between cells (from direct contact to several $100 \mu \mathrm{m}$ ), which may limit cell-cell communication via highly reactive and thus instable oxidants. However, effects of oxidation (intracellular or extracellular) of secreted proteins, such as extracellular matrix proteins or growth factors, could be studied and may provide relevant information on redox-mediated tissue homeostasis. Alternative technologies for intra-tissue assessment of oxidation were recently developed (Fujikawa et al. 2016) and rely on the arrest of the redox state of the roGFP-biosensor by the membrane-permeable thiol-alkylating agent (NEM). First experiments in human EHMs suggest that this method would indeed be applicable to study the redox state histologically. This together with the possibility for in tissue fluorescence analyses by confocal ( $\sim 50 \mu \mathrm{m}$ penetration depth) and 2-photon ( $200 \mu \mathrm{m})$ microscopy should allow for comprehensive in tissue phenotyping of redox mechanisms and their association with heart muscle function. 


\section{Summary and outlook}

This study introduces the use of the glutathione redox sensor Grx1-roGFP2 as a tool to monitor responses to oxidative and reductive challenges in a human heart muscle specific context. Redox challenges are considered key contributors to physiological and pathological processes in the heart. In addition, pharmacological interventions often influence the cellular redox state. With the introduction of a human Grx1roGFP2 engineered heart muscle (EHM) model we anticipate to be able to contribute to the definition of mechanisms underlying wanted and unwanted drug effects as well as cardiac disease progression. A key observation of this study was the finding of a differential glutathione redox potential in cardiomyocyte and fibroblasts. Thus, drugs with oxidant and antioxidant activity may elicit sometimes even mechanistically opposing functions in the different myocardial cell compartments. Future studies will investigate this finding in more detail and take advantage of the possibility to reconstitute EHM with defined cell types with or without a genetically encoded redox sensor. Targeting subcellular compartments in a cell type specific context will further open experimental possibilities to decipher redox mechanisms and may eventually contribute to the development of cell and cell compartment targeted interventions for the treatment of heart failure. 


\section{Appendix 1}

\section{Reagents for cloning}

\section{LB-medium}

$\begin{array}{ll}10 \mathrm{~g} & \text { Bacto-Tryptone } \\ 5 \mathrm{~g} & \text { Bacto Yeast Extract } \\ 10 \mathrm{~g} & \mathrm{NaCl}\end{array}$

All the components were dissolved and filled up to $1 \mathrm{~L}$ of $\mathrm{dH}_{2} \mathrm{O}$. $\mathrm{pH}$ was adjusted to 7.4. The medium was stored at $4^{\circ} \mathrm{C}$ for up to 3 weeks.

Ampicillin

To prepare a stock of $100 \mathrm{mg} / \mathrm{mL} 200 \mathrm{mg}$ were dissolved in $2 \mathrm{ml}$ of $\mathrm{dH}_{2} \mathrm{O}$. The stock solution was aliquoted in $500 \mu \mathrm{l}$ and stored at $-20^{\circ} \mathrm{C}$.

\section{LB-agarose plate (with ampicillin resistance)}

\section{$7.5 \mathrm{~g} \quad$ Agar}

$500 \mathrm{ml} \quad$ LB-medium

$500 \mu \mathrm{l} \quad$ Ampicillin stock $(100 \mathrm{mg} / \mathrm{mL})$

The agar was dissolved in LB-medium and autoclaved. After cooling down to $50^{\circ} \mathrm{C}$, ampicillin stock was added and the solution was poured into $10 \mathrm{~cm}$ petri dishes. Following the hardening of the agar, the plates were stored at $4{ }^{\circ} \mathrm{C}$. 


\section{Reagents for DNA gel electrophoresis}

\section{$\underline{50 \times \mathrm{TAE}}$}

$242 \mathrm{~g} \quad$ Tris-Base

$100 \mathrm{ml} \quad$ EDTA $(0.5 \mathrm{~mol} / \mathrm{L} ; \mathrm{pH} 8.0)$

$57.1 \mathrm{ml} \quad$ Glacial acetic acid

The ingredients were dissolved in $800 \mathrm{ml}$ of $\mathrm{dH}_{2} \mathrm{O}$, adjusted $\mathrm{pH}$ at 8.5 and then filled up to $1 \mathrm{~L}$ of $\mathrm{dH}_{2} \mathrm{O}$. For gel electrophoresis, $1 \times \mathrm{TAE}$ buffer was used (1:50 dilution).

6x DNA loading buffer (2 colors)

$75 \mathrm{mg} \quad$ Bromophenol-Blue

$25 \mathrm{mg} \quad$ Xylene cyanol

$100 \mathrm{ml} \quad$ Glycerol (30\%)

\section{$1 \%$ agarose gel}

$1 \mathrm{~g}$ of Agarose UltraPureTM powder (AppliChem) was boiled in $100 \mathrm{ml}$ TAE buffer until the powder was completely dissolved. After cooling, $0.2 \mu \mathrm{g} / \mathrm{ml}$ ethidium bromide (EtBR; Sigma-Aldrich) was added into the gel solution, following by a gentle swirl. The homogeneous solution was added into already prepared casting trays. 


\section{Reagents and media for cell culture}

Activin A stock solution

(Manufacturer's protocol)

To prepare a stock solution of $10 \mu \mathrm{g} / \mathrm{ml}$ dissolve activin A (Recombinant Human/Mouse/Rat Activin A protein; R\&D Systems \#338-AC) in 1x PBS with $0.1 \%$ human recombinant serum albumin (HAS) (Sigma-Aldrich \#A9731). Aliquots were stored in $-20^{\circ} \mathrm{C}$.

\section{BMP-4 stock solution}

(Manufacturer's protocol)

To prepare a stock solution of $10 \mu \mathrm{g} / \mathrm{ml}$ dissolve BMP-4 (Recombinant Human BMP4; Sigma-Aldrich \#314-BP) in 1x PBS with 0.1\% HSA (Sigma-Aldrich \#A9731). Aliquots were stored in $-20^{\circ} \mathrm{C}$.

\section{IWP-4 stock solution}

(Manufacturer's protocol)

To prepare a stock solution of $5 \mathrm{mmol} / \mathrm{L}$ dissolve IWP-4 (Stemolecule ${ }^{\mathrm{TM}} \mathrm{Wnt}$ inhibitor IWP-4; Stemgent \#04-0036) in DMSO. Aliquots were stored in $-20^{\circ} \mathrm{C}$.

\section{$\underline{\text { CHIR stock solution }}$}

(Manufacturer's protocol)

To prepare a stock solution of $10 \mathrm{mmol} / \mathrm{L}$ dissolve CHIR (Stemolecule ${ }^{\mathrm{TM}} \mathrm{CHIR99021}$; Stemgent \#04-0004) in DMSO. Aliquots were stored in $-20^{\circ} \mathrm{C}$.

\section{bFGF stock solution}

(Manufacturer's protocol)

To prepare a stock solution of $10 \mu \mathrm{g} / \mathrm{ml}$ dissolve bFGF (Recombinant Human FGFbasic [154 aa]; Peprotech \#AF-100-18B) in 1x PBS with 0.1\% HSA (Sigma-Aldrich \#A9731). Aliquots were stored in $-20^{\circ} \mathrm{C}$.

IGF-1 stock solution

(Manufacturer's protocol) 
To prepare a stock solution of $100 \mu \mathrm{g} / \mathrm{ml}$ dissolve IGF (Recombinant Human IGF-1; Peprotech \#AF-100-11) in 1x PBS with 0.1\% HSA (Sigma-Aldrich \#A9731). Aliquots were stored in $-20^{\circ} \mathrm{C}$.

\section{VEGF stock solution}

(Manufacturer's protocol)

To prepare a stock solution of $5 \mu \mathrm{g} / \mathrm{ml}$ dissolve VEGF (Animal-Free Recombinant Human VEGF [165 aa]; Peprotech \#AF-100-20) in 1x PBS with 0.1\% HSA (SigmaAldrich \#A9731). Aliquots were stored in $-20^{\circ} \mathrm{C}$.

\section{$\underline{\text { TGF-B1 stock solution }}$}

(Manufacturer's protocol)

To prepare a stock solution of $5 \mu \mathrm{g} / \mathrm{ml}$ dissolve TGF-B1 (Recombinant Human TGFB1 [CHO cell derived]; Peprotech \#AF-100-21C) in 1x PBS with $0.1 \%$ HSA (SigmaAldrich \#A9731). Aliquots were stored in $-20^{\circ} \mathrm{C}$.

\section{Polybrene stock solution}

To prepare $1 \mathrm{mg} / \mathrm{mL}$ stock $10 \mathrm{mg}$ of hexadimethine bromide (Sigma Aldrich) was dissolved in $10 \mathrm{ml}$ of PBS or $0.9 \% \mathrm{NaCl}$ solution (ready purchased). The solution was filtered and stored at $4{ }^{\circ} \mathrm{C}$. It was used for transduction at a final concentration of 8 $\mu \mathrm{g} / \mathrm{ml}$.

\section{Ascorbic acid stock solution}

To prepare a stock solution of $300 \mathrm{mmol} / \mathrm{L}$ dissolve $0.87 \mathrm{~g}$ of L-ascorbic acid 2phosphate sesquimagnesium salt hydrate (Sigma-Aldrich \#A8960) in $10 \mathrm{ml}$ of $\mathrm{dH}_{2} \mathrm{O}$ and sterile filtrate it. Aliquots were stored in $-20^{\circ} \mathrm{C}$.

\section{0x RPMI}

RPMI powder (RPMI 1640 medium powder; Gibco \#52800-035) was dissolved in 10 $\mathrm{ml}$ of $\mathrm{dH}_{2} \mathrm{O}$, sterile filtered and stored at $4{ }^{\circ} \mathrm{C}$.

\section{$\underline{\text { 2x RPMI }}$}

$2 \mathrm{ml} \quad 10 \times \mathrm{RPMI}$ 
$\begin{array}{ll}0.8 \mathrm{ml} & \left.\mathrm{B}-27^{(}\right) \text {Supplement }(50 x ; G i b c o ~ \# 17504-044) \\ 0.2 \mathrm{ml} & \mathrm{P} / \mathrm{S}(100 x . G i b c o \text { \#15140-122) } \\ 7 \mathrm{ml} & \mathrm{H}_{2} \mathrm{O}\end{array}$

Sterile filtered solution was stored at $4{ }^{\circ} \mathrm{C}$ until further use

Accutase digestion solution

Mix Accutase (Millipore SCR005) with 0.125 \% Trypsin (diluted 1:20 from Trypsin 2.5 \% stock; Gibco 15090-046) and add 20 rg/ml DNase I.

\section{Collagenase digestion solution}

Dissolve $500 \mathrm{mg}$ of collagenase (Collagenase from Clostridium histolyticum for general use, Type I; Sigma-Aldrich \# C0130) in $250 \mathrm{ml}$ of $1 \times \mathrm{PBS}$ with $\mathrm{Ca}^{2+} / \mathrm{Mg}^{2+}$ and $20 \%$ FBS (Gibco \#10270). Aliquots were stored in $-20^{\circ} \mathrm{C}$.

\section{HES2-CM serum-free medium}

$500 \mathrm{ml} \quad$ RPMI medium 1640.GlutaMAXTM (Gibco \#61870-010)

$5.2 \mathrm{ml} \quad \mathrm{P} / \mathrm{S}(100 \mathrm{x}$;Gibco \#15140-122)

$10 \mathrm{ml} \quad$ B-27® Supplement (50x;Gibco \#17504-044)

$335 \mu \mathrm{l} \quad$ Ascorbic acid stock solution (300 $\mathrm{mmol} / \mathrm{L})$

\section{HFF medium}

$500 \mathrm{ml} \quad$ RPMI medium 1640.GlutaMAX ${ }^{\text {TM }}$ (Gibco \#61870-010)

$88.6 \mathrm{ml} \quad$ FBS (Gibco \#10270)

$6.2 \mathrm{ml} \quad$ Pen/Strep (100x;Gibco \#15140-122)

HFF conditioned medium

$50 \mathrm{ml} \quad$ HESC medium

$25 \mu \mathrm{l} \quad$ bFGF (final concentration $5 \mathrm{ng} / \mathrm{ml}$; Miltenyi Biotech)

\section{HESC medium}

$39.5 \mathrm{ml} \quad$ KO DMEM medium (Invitrogen. \#10829)

$10 \mathrm{ml} \quad$ KSR (Knockout Serum Replacement; Invitrogen. \#10828)

$0.5 \mathrm{ml} \quad \mathrm{P} / \mathrm{S}$ (100x;Gibco \#15140-122) 


$\begin{array}{ll}0.5 \mathrm{ml} & \text { MEM-NEAA (100x; Gibco \#111450-035) } \\ 0.5 \mathrm{ml} & \text { L-Glutamine (200 mmol/L; Gibco \#25030-024) } \\ 50 \mu \mathrm{l} & \text { bFGF (final concentration } 10 \mathrm{ng} / \mathrm{ml} \text {; Miltenyi Biotech) }\end{array}$

\section{HESC conditioned medium}

$25 \mathrm{ml} \quad$ RPMI medium 1640, GlutaMAX ${ }^{\text {TM }}$ (Gibco \#61870-010)

$25 \mathrm{ml} \quad$ FBS (Gibco \#10270)

$50 \mu \mathrm{l} \quad$ P/S (100x; Gibco \#15140-122)

\section{Cardiac specification medium}

$50 \mathrm{ml} \quad$ HES2-CM serum-free medium

$50 \mu \mathrm{l} \quad$ IWP4 stock solution (final concentration $5 \mu \mathrm{mol} / \mathrm{L}$ )

\section{Selection medium}

$49.5 \mathrm{ml}$ RPMI 1640 (without glucose, without-glutamine; Biological Industries/WKS Labor diagnostik \#01-101-1A)

$0.25 \mathrm{ml}$ Sodium Lactate (final concentration $2.2 \mathrm{mmol} / \mathrm{L}$; Sigma-Aldrich 71723)

$0.5 \mathrm{ml} \quad \mathrm{P} / \mathrm{S}(100 \mathrm{x}$; Gibco \#15140-122)

$0.1 \mathrm{ml} \quad$ 2-mercaptoethanol (50 mmol/L; Invitrogen \#31350010)

TSA culture medium

$500 \mathrm{ml} \quad$ DMEM medium (Gibco \#61965-026)

$50 \mathrm{ml} \quad$ FBS (Gibco \#10270)

$5.5 \mathrm{ml} \quad \mathrm{P} / \mathrm{S}$ (100x;Gibco \#15140-122)

TSA low serum medium

$500 \mathrm{ml} \quad$ DMEM medium (Gibco \#61965-026)

$25 \mathrm{ml} \quad$ FBS (Gibco \#10270)

$5.2 \mathrm{ml} \quad \mathrm{P} / \mathrm{S}$ (100x;Gibco \#15140-122) 
EHM medium (incomplete)

$500 \mathrm{ml} \quad$ IMDM GlutaMAX

$5.5 \mathrm{ml} \quad \mathrm{P} / \mathrm{S}(100 \mathrm{x}$;Gibco \#15140-122)

$5.5 \mathrm{ml} \quad$ L-Glutamine (200 mmol/L; Gibco \#25030-024)

$5.5 \mathrm{ml} \quad$ MEM-NEAA (100x; Gibco\#111450-035)

$500 \mu \mathrm{l} \quad$ Ascorbic acid (stock solution: $300 \mathrm{mmol} / \mathrm{L}$ )

EHM medium (complete)

$50 \mathrm{ml} \quad$ EHM medium (incomplete)

$2 \mathrm{ml} \quad$ B-27® minus insulin (50x;Gibco \#A18956-01)

$50 \mu \mathrm{l} \quad$ bFGF stock solution (final concentration $10 \mathrm{ng} / \mathrm{ml}$ )

$50 \mu \mathrm{l} \quad$ IGF-1 stock solution (final concentration $100 \mathrm{ng} / \mathrm{ml}$ )

$50 \mu \mathrm{l} \quad$ VEGF stock solution (final concentration $5 \mathrm{ng} / \mathrm{ml}$ )

\section{Freezing medium}

90\% $\quad$ FBS (Gibco \#10270)

10\% DMSO (Sigma-Aldrich \#276855)

Cell pellets were resuspended in FBS mixture with DMSO and then transferred into cryovials. The cryovials were stored at $-152{ }^{\circ} \mathrm{C}$. 


\section{Reagents for redox measurement}

\section{$\underline{\mathrm{KCl} \text { stock solution }}$}

To prepare $1 \mathrm{~mol} / \mathrm{L}$ stock solution, $18.64 \mathrm{~g}$ of $\mathrm{KCl}$ was dissolved and filled up to 250 $\mathrm{ml}$ of $\mathrm{dH}_{2} \mathrm{O}$. The solution was stored at RT.

\section{$\underline{\mathrm{MgCl}_{2}}$}

To prepare a $1 \mathrm{~mol} / \mathrm{L}$ stock solution, $50.82 \mathrm{~g}$ of $\mathrm{MgCl}_{2}$ was dissolved and filled up to $250 \mathrm{ml}$ of $\mathrm{dH}_{2} \mathrm{O}$. The solution was stored at RT.

\section{$\underline{\mathrm{CaCl} 2}$}

To prepare $1 \mathrm{~mol} / \mathrm{L}$ stock solution, $26.75 \mathrm{~g}$ of $\mathrm{CaCl}_{2}$ was dissolved and filled up to $250 \mathrm{ml}$ of $\mathrm{dH}_{2} \mathrm{O}$. The solution was stored at RT.

\section{Imaging buffer}

$144 \mathrm{mmol} / \mathrm{L} \quad \mathrm{NACl}$

$5.4 \mathrm{mmol} / \mathrm{L} \quad \mathrm{KCl}$

$1 \mathrm{mmol} / \mathrm{L} \quad \mathrm{MgCl}_{2}$

$10 \mathrm{mmol} / \mathrm{L} \quad$ HEPES

$1 \mathrm{mmol} / \mathrm{L} \quad \mathrm{CaCl}_{2}$

For the preparation of the imaging buffer, the ingredients listed above $(1 \mathrm{mmol} / \mathrm{L}$ or 2 $\mathrm{mmol} / \mathrm{L}$ of $\mathrm{CaCl}_{2}$ for $\mathrm{CMs}$ and HFFs, respectively) were dissolved in $\mathrm{dH}_{2} \mathrm{O}$ and $\mathrm{pH}$ gradient was adjusted to 7.3. The buffer was stored at RT.

\section{$\underline{\mathrm{H}}_{2} \underline{\mathrm{O}}_{2}$ solution}

The stock solution $30 \% \mathrm{H}_{2} \mathrm{O}_{2}(\mathrm{Mw}=34.01 \mathrm{~g} / \mathrm{mol}$; Sigma-Aldrich \#7722-84-1) was equivalent to $10 \mathrm{~mol} / \mathrm{L}(\mathrm{d}=1.11 \mathrm{~g} / \mathrm{ml})$ and was store at $4{ }^{\circ} \mathrm{C}$. Dilutions of $(0.1-1,000$ $\mu \mathrm{mol} / \mathrm{L})$ were freshly prepared for each experiment.

\section{Diamide stock solution}

To prepare a stock solution of $1 \mathrm{~mol} / \mathrm{L}, 1 \mathrm{~g}$ of diamide $(\mathrm{Mw}=172.19 \mathrm{~g} / \mathrm{mol}$; Sigma Aldrich \#10465-78-8) was diluted into $5.8 \mathrm{ml} \mathrm{dH}_{2} \mathrm{O}$. The solution was filtered, aliquot 
and stored at $-20^{\circ} \mathrm{C}$. Dilutions of $(1-1,000 \mu \mathrm{mol} / \mathrm{L})$ were freshly prepared for each experiment.

\section{DTT stock solution}

To prepare a stock solution of $1 \mathrm{~mol} / \mathrm{L}$ dissolve $1.54 \mathrm{~g}$ of DTT (Sigma Aldrich \#3483-

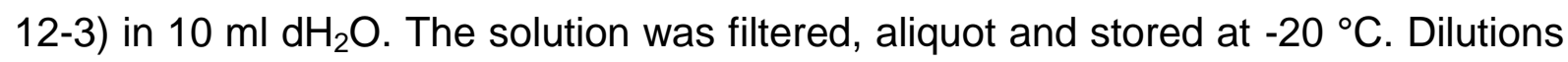
of $(0.1-1 \mathrm{mmol} / \mathrm{L})$ were freshly prepared for each experiment.

\section{Angiotensin II stock solution}

To prepare a stock solution of $5 \mu \mathrm{mol} / \mathrm{L}$ dissolve $1 \mathrm{mg}$ of Angitensin II (Sigma-Aldrich \#4474-91-3) into $40 \mu \mathrm{l}$ of $\mathrm{dH}_{2} \mathrm{O}$. The solution was further dissolved in $200 \mathrm{ml}$ of $\mathrm{dH}_{2} \mathrm{O}$ and aliquots were stored at $-20{ }^{\circ} \mathrm{C}$. Dilutions of $(0.1-1,000 \mathrm{nmol} / \mathrm{L})$ were freshly prepared for each experiment.

\section{Levosimendan stock solution}

To prepare a stock solution of $1 \mathrm{mmol} / \mathrm{L}$ dissolve $5 \mathrm{mg}$ of Levosimendan (SigmaAldrich 141505-33-1) in $178 \mu$ of DMSO. The solution was thoroughly dissolved into $17.8 \mathrm{ml}$ of $\mathrm{dH}_{2} \mathrm{O}$ and aliquots were stored at $-20^{\circ} \mathrm{C}$. Dilutions of $(0.1-10 \mu \mathrm{mol} / \mathrm{L})$ were freshly prepared for each experiment.

\section{Omecamtiv mecarbil (CK-1827452) stock solution}

To prepare a stock solution of $1 \mathrm{mmol} / \mathrm{L}$ dissolve $5 \mathrm{mg}$ of omecamtiv mecarbil (CK1827452; AdooQ Bioscience \#A11206) in $124.6 \mu \mathrm{l}$ of DMSO. The solution was thoroughly dissolved into $12.46 \mathrm{ml}$ of $\mathrm{dH}_{2} \mathrm{O}$ and aliquots were stored at $-20{ }^{\circ} \mathrm{C}$. Dilutions of $(0.1-10 \mu \mathrm{mol} / \mathrm{L})$ were freshly prepared for each experiment. 


\section{Reagents for Immunoblotting}

6x Laemmli loading buffer (non-reducing)

$3 \mathrm{ml} \quad$ Tris- $\mathrm{HCl} 0.5 \mathrm{~mol} / \mathrm{L}(\mathrm{pH} \mathrm{6.8})$

$1.2 \mathrm{~g} \quad$ SDS

$0.75 \mathrm{ml} \quad$ Bromophenol blue $(0.5 \%)$

$1.5 \mathrm{ml} \quad$ Glycerin (100\%)

All the ingredients were dissolved and filled up to $9 \mathrm{ml}$ with $\mathrm{H}_{2} \mathrm{O}$. For a reducing gel, $150 \mu \mathrm{l} 2-ß$-Mercaptoethanol was added into the final solution. Aliquots were stored at $-20^{\circ} \mathrm{C}$.

\section{$1.5 \mathrm{~mol} / \mathrm{L}$ Tris- $\mathrm{HCl} \mathrm{pH} 8.8$}

$90.85 \mathrm{~g}$ of Tris- $\mathrm{HCl}$ was dissolved and filled up to $500 \mathrm{ml}$ of $\mathrm{dH}_{2} \mathrm{O}$. $\mathrm{pH}$ was adjusted to $\mathrm{pH} 8.8$.

\section{$\underline{0.5 \mathrm{~mol} / \mathrm{L} \text { Tris- } \mathrm{HCl} \mathrm{pH} 6.8}$}

$30.28 \mathrm{~g}$ of Tris- $\mathrm{HCl}$ was dissolved and filled up to $500 \mathrm{ml}$ of $\mathrm{dH} 2 \mathrm{O}$. $\mathrm{pH}$ was adjusted to 6.8

\section{Preparation of SDS-PAGE}

Separating gel

\begin{tabular}{|c|c|c|c|c|c|}
\hline $\begin{array}{c}\text { Volume (mI) } \\
\text { for } \mathbf{2} \text { gels }\end{array}$ & $\mathbf{6 \%}$ & $\mathbf{8 \%}$ & $\mathbf{1 0 \%}$ & $\mathbf{1 2 \%}$ & $\mathbf{1 5 \%}$ \\
\hline Protein size (KDa) & $150-50$ & $105-40$ & $90-25$ & $60-20$ & $45-10$ \\
\hline $\mathbf{H}_{\mathbf{2}} \mathbf{O}$ & 5.3 & 4.6 & 4.0 & 3.3 & 2.3 \\
\hline $\mathbf{3 0} \%$ Acrylamide & 2.0 & 2.7 & 3.3 & 4.0 & 5.0 \\
\hline $\mathbf{1 . 5} \mathbf{M}$ Tris pH 8.8 & 2.5 & 2.5 & 2.5 & 2.5 & 2.5 \\
\hline $\mathbf{1 0} \%$ SDS & 0.1 & 0.1 & 0.1 & 0.1 & 0.1 \\
\hline $\mathbf{1 0} \%$ APS & 0.1 & 0.1 & 0.1 & 0.1 & 0.1 \\
\hline TEMED & 0.008 & 0.006 & 0.004 & 0.004 & 0.004 \\
\hline
\end{tabular}


Stacking/collecting gel

\begin{tabular}{|c|c|}
\hline Volume (ml) & $\mathbf{4}$ gels \\
\hline $\mathbf{H}_{2} \mathbf{O}$ & 5.6 \\
\hline $\mathbf{3 0} \%$ Acrylamid & 0.85 \\
\hline $\mathbf{0 . 5} \mathbf{M}$ Tris $\mathbf{~ p H ~} \mathbf{6 . 8}$ & 2.5 \\
\hline $\mathbf{1 0} \% \mathbf{S D S}$ & 0.1 \\
\hline $\mathbf{1 0} \%$ APS & 0.1 \\
\hline TEMED & 0.01 \\
\hline
\end{tabular}

10x Electrophoresis / Running buffer (pH 8.3 - 8.7)

$60.6 \mathrm{~g} \quad$ Tris-Base

$288 \mathrm{~g} \quad$ Glycine

$20 \mathrm{~g} \quad$ SDS

All the ingredients were dissolved and fully mixed in $1 \mathrm{LdH}_{2} \mathrm{O}$ and filled up to $2 \mathrm{~L}$ of $\mathrm{dH}_{2} \mathrm{O}$. For every running of gel electrophoresis, $1 \mathrm{x}$ running buffer (1:10 dilution) was freshly prepared.

10x Transfer buffer

$60.5 \mathrm{~g} \quad$ Tris-Base

$288 \mathrm{~g} \quad$ Glycine

The ingredients were fully dissolved in $2 \mathrm{LdH}_{2} \mathrm{O}$.

$\underline{1 x \text { Transfer buffer }}$

$200 \mathrm{ml} \quad 10 \times$ Transfer buffer

$400 \mathrm{ml} \quad$ Methanol

The complete solution was filled up to $2 \mathrm{~L}$ of $\mathrm{dH}_{2} \mathrm{O}$.

10x TBS stock solution

$121.14 \mathrm{~g} \quad$ Tris-Base

$175.32 \quad \mathrm{NaCl}$ 
The ingredients were dissolved in $1.5 \mathrm{~L}$ of $\mathrm{H}_{2} \mathrm{O}$ and stirred until fully dissolved. $\mathrm{pH}$ was adjusted to 7.5 by using fuming $\mathrm{HCl}$. The final solution was filled up $2 \mathrm{~L}$ of $\mathrm{H}_{2} \mathrm{O}$.

\section{$\underline{1 x \text { TBST solution }}$}

$100 \mathrm{ml} \quad 10 \times \mathrm{TBS}$

$900 \mathrm{ml} \quad \mathrm{H} 2 \mathrm{O}$

$1 \mathrm{ml} \quad$ Tween 20

All the ingredients were mixed until fully dissolved.

\section{Preparation of PVDF membrane}

Activation of PVDF membrane was done by immersing it for $10 \mathrm{sec}$ in $100 \%$ Methanol, followed by a washing step for $5 \mathrm{~min}$ in $\mathrm{dH}_{2} \mathrm{O}$ and equilibrating it for $5-10$ min in transfer buffer.

\section{Ponceau staining}

Membranes were incubated for 1-3 min at RT in Ponceau S-solution (Applichem) and then rinsed with $\mathrm{dH}_{2} \mathrm{O}$ to remove the extra red solution. After the staining, protein bands appear red colored and this color was rinsed by TBST solution. 


\section{Reagents for isomeric measurement}

\section{$\underline{\mathrm{CaCl}_{2}} \underline{\text { stock solution }}$}

To prepare stock solution of $2.25 \mathrm{~mol} / \mathrm{L}$ dissolve $165.57 \mathrm{~g}$ of $\mathrm{CaCl}_{2} \times 2 \mathrm{H}_{2} \mathrm{O}$ in $500 \mathrm{ml}$ of $\mathrm{dH}_{2} \mathrm{O}$. The stock was stored at $4{ }^{\circ} \mathrm{C}$.

\section{$\underline{\mathrm{MgCl}_{2}}$ stock solution}

To prepare a stock solution of $1.05 \mathrm{~mol} / \mathrm{L}$ dissolve $106.83 \mathrm{~g}$ of $\mathrm{MgCl}_{2} \times 6 \mathrm{H}_{2} \mathrm{O}$ in 500 $\mathrm{ml}$ of $\mathrm{dH}_{2} \mathrm{O}$. The stock was stored at $4{ }^{\circ} \mathrm{C}$.

\section{Stock I solution ( $0.2 \mathrm{mmol} / \mathrm{L}$ Calcium)}

$175 \mathrm{~g} \mathrm{NaCl}$

$10 \mathrm{~g} \quad \mathrm{KCl}$

$2.22 \mathrm{ml} \quad \mathrm{CaCl}_{2}$ stock solution $(2.25 \mathrm{~mol} / \mathrm{L})$

$25 \mathrm{ml} \quad \mathrm{MgCl}_{2}$ stock solution (1.05 mol/L)

The ingredients were dissolved and filled up till $1 \mathrm{~L}$ with $\mathrm{dH}_{2} \mathrm{O}$. The stock was stored at $4{ }^{\circ} \mathrm{C}$.

Stock II solution

$50 \mathrm{~g}$ of $\mathrm{NAHCO}_{3}$ was dissolved into $1 \mathrm{~L}$ of $\mathrm{dH}_{2} \mathrm{O}$ and stored at $4{ }^{\circ} \mathrm{C}$.

\section{Stock III solution}

$5.8 \mathrm{~g}$ of $\mathrm{NAH}_{2} \mathrm{PO}_{4}$ was dissolved into $1 \mathrm{~L}$ of $\mathrm{dH}_{2} \mathrm{O}$ and stored at $4{ }^{\circ} \mathrm{C}$.

\section{Tyrode's solution}

$200 \mathrm{ml} \quad$ Stock I solution $(0.2 \mathrm{mmol} / \mathrm{L}$ Calcium $)$

$190 \mathrm{ml} \quad$ Stock II solution

$50 \mathrm{ml} \quad$ Stock III solution

$5 \mathrm{~g} \quad$ D-Glucose

$500 \mathrm{mg} \quad$ Ascorbic acid

The ingredients were dissolved and filled up to $5 \mathrm{~L}$ of $\mathrm{dH}_{2} \mathrm{O}$. The Tyrode's solution was prepared before the contraction force measurement. 


\section{Reagents for Immunstaining and Flow Cytometry}

Permealizing blocking buffer

$26.3 \mathrm{ml} \quad$ Goat serum

$5.26 \mathrm{~g} \quad$ Bovine serum albumin (BSA; Sigma-Aldrich \#A3311)

2.63 Triton $\mathrm{X}-100$

The ingredients were dissolved and filled up to $500 \mathrm{ml}$ of $1 \mathrm{x}$ PBS. The solution was stored at $4^{\circ} \mathrm{C}$.

Non-permealizing blocking buffer

The buffer was prepared by adding 5\% of FBS (Gibco \#10270) into 1x PBS solution and stored at $4^{\circ} \mathrm{C}$. 


\section{Appendix 2}

\begin{tabular}{|c|c|c|}
\hline Cell line & Genetic Background & Source \\
\hline TSA201 & $\begin{array}{c}\text { Human Embryonic kidney immortalized } \\
\text { by an adenovirus serotype } 5, \text { SV40 } \\
\text { large T-antigen }\end{array}$ & $\begin{array}{l}\text { Cells used for lentiviral } \\
\text { production }\end{array}$ \\
\hline HFFs & Human Foreskin Fibroblasts. wild type & $\begin{array}{l}\text { Purchased from American } \\
\text { Type Culture Collection } \\
\text { (SCRC-1041; ATCC) }\end{array}$ \\
\hline $\begin{array}{l}\text { HFFs } \\
\text { (pGIPZ) }\end{array}$ & $\begin{array}{l}\text { Human Foreskin Fibroblasts with } \\
\text { lentiviral transduction of pGIPZ }\end{array}$ & $\begin{array}{l}\text { Transgenic modification via } \\
\text { lentiviral transduction }\end{array}$ \\
\hline roGFP2-HFF & $\begin{array}{c}\text { Human Foreskin Fibroblasts with } \\
\text { lentiviral transduction of pGIPZ-CMV- } \\
\text { Grx1-roGFP2 }\end{array}$ & $\begin{array}{l}\text { Transgenic modification via } \\
\text { lentiviral transduction }\end{array}$ \\
\hline HES2-RFP & $\begin{array}{c}\text { HES2 line (Embryonic Stem cell } \\
\text { International) (Reubinoff et al. 2000) } \\
\text { including tdRFP knock-in in ROSA26 } \\
\text { locus }\end{array}$ & $\begin{array}{l}\text { Kindly provided by Gordon } \\
\text { Keller (Irion et al. 2007) }\end{array}$ \\
\hline $\begin{array}{l}\text { HES2-RFP } \\
\text { CM }\end{array}$ & $\begin{array}{l}\text { Human cardiomyocytes derived from } \\
\text { HES2-RFP }\end{array}$ & See section 2.1.3.1 \\
\hline $\begin{array}{l}\text { HES2-RFP } \\
\text { CM (pGIPZ) }\end{array}$ & $\begin{array}{l}\text { HES2-RFP CM with lentiviral } \\
\text { transduction of pGIPZ }\end{array}$ & $\begin{array}{l}\text { Transgenic modification via } \\
\text { lentiviral transduction }\end{array}$ \\
\hline $\begin{array}{l}\text { rOGFP2- } \\
\text { HES2-RFP } \\
\text { CM }\end{array}$ & $\begin{array}{c}\text { HES2-RFP CM with lentiviral } \\
\text { transduction of pGIPZ-CMV-Grx1- } \\
\text { roGFP2 }\end{array}$ & $\begin{array}{l}\text { Transgenic modification via } \\
\text { lentiviral transduction }\end{array}$ \\
\hline
\end{tabular}

Table 12: Cell lines. 


\section{Bibliography}

1. Albrecht SC, Barata AG, Grosshans J, Teleman AA, Dick TP (2011) In vivo mapping of hydrogen peroxide and oxidized glutathione reveals chemical and regional specificity of redox homeostasis. Cell Metab. 14:819-29.

2. Aller I, Rouhier N, Meyer AJ (2013) Development of roGFP2-derived redox probes for measurement of the glutathione redox potential in the cytosol of severely glutathione-deficient rml1 seedlings. Front Plant Sci. 4:506.

3. Amin JK, Xiao L, Pimental DR, Pagano PJ, Singh K, Sawyer DB, Colucci WS (2001) Reactive oxygen species mediate alpha-adrenergic receptor-stimulated hypertrophy in adult rat ventricular myocytes. J Mol Cell Cardiol. 33:131-9.

4. Belousov VV, Fradkov AF, Lukyanov KA, Staroverov DB, Shakhbazov KS, Terskikh AV, Lukyanov S (2006) Genetically encoded fluorescent indicator for intracellular hydrogen peroxide. Nat Methods. 3:281-6.

5. Bienert GP, Schjoerring JK, Jahn TP (2006) Membrane transport of hydrogen peroxide. Biochim Biophys Acta. 1758:994-1003.

6. Bilan DS, Pase L, Joosen L, Gorokhovatsky AY, Ermakova YG, Gadella TW, Grabher C, Schultz C, Lukyanov S, Belousov VV (2013) HyPer-3: a genetically encoded $\mathrm{H}(2) \mathrm{O}(2)$ probe with improved performance for ratiometric and fluorescence lifetime imaging. ACS Chem Biol. 8:535-42.

7. Bjornberg O, Ostergaard H, Winther JR (2006) Mechanistic insight provided by glutaredoxin within a fusion to redox-sensitive yellow fluorescent protein. Biochemistry. 45:2362-71.

8. Bolli R (1998) Causative role of oxyradicals in myocardial stunning: a proven hypothesis. A brief review of the evidence demonstrating a major role of reactive 
oxygen species in several forms of postischemic dysfunction. Basic Res Cardiol. 93:156-62.

9. Bomprezzi R (2015) Dimethyl fumarate in the treatment of relapsing-remitting multiple sclerosis: an overview. Ther Adv Neurol Disord. 8:20-30.

10. Bouzegrhane F, Thibault $G$ (2002) Is angiotensin II a proliferative factor of cardiac fibroblasts? Cardiovasc Res. 53:304-12.

11. Bradford MM (1976) A rapid and sensitive method for the quantitation of microgram quantities of protein utilizing the principle of protein-dye binding. Anal Biochem. 72:248-54.

12. Brown DA, Perry JB, Allen ME, Sabbah HN, Stauffer BL, Shaikh SR, Cleland JG, Colucci WS, Butler J, Voors AA, Anker SD, Pitt B, Pieske B, Filippatos G, Greene SJ, Gheorghiade M (2016) Expert consensus document: Mitochondrial function as a therapeutic target in heart failure. Nat Rev Cardiol.

13. Bujak M, Frangogiannis NG (2007) The role of TGF-beta signaling in myocardial infarction and cardiac remodeling. Cardiovasc Res. 74:184-95.

14. Burgoyne JR, Mongue-Din H, Eaton P, Shah AM (2012) Redox signaling in cardiac physiology and pathology. Circ Res. 111:1091-106.

15. Cesselli D, Jakoniuk I, Barlucchi L, Beltrami AP, Hintze TH, Nadal-Ginard B, Kajstura J, Leri A, Anversa P (2001) Oxidative stress-mediated cardiac cell death is a major determinant of ventricular dysfunction and failure in dog dilated cardiomyopathy. Circ Res. 89:279-86.

16. Charles RL, Eaton $P$ (2008) Redox signalling in cardiovascular disease. Proteomics Clin Appl. 2:823-36.

17. Chen CA, Wang TY, Varadharaj S, Reyes LA, Hemann C, Talukder MA, Chen YR, Druhan LJ, Zweier JL (2010) S-glutathionylation uncouples eNOS and regulates its cellular and vascular function. Nature. 468:1115-8. 
18. Cheng TH, Cheng PY, Shih NL, Chen IB, Wang DL, Chen JJ (2003) Involvement of reactive oxygen species in angiotensin II-induced endothelin-1 gene expression in rat cardiac fibroblasts. J Am Coll Cardiol. 42:1845-54.

19. Colston JT, de la Rosa SD, Strader JR, Anderson MA, Freeman GL (2005) $\mathrm{H} 2 \mathrm{O} 2$ activates Nox4 through PLA2-dependent arachidonic acid production in adult cardiac fibroblasts. FEBS Lett. 579:2533-40.

20. Dao VT, Casas Al, Maghzal GJ, Seredenina T, Kaludercic N, RobledinosAnton N, Di Lisa F, Stocker R, Ghezzi P, Jaquet V, Cuadrado A, Schmidt HH (2015) Pharmacology and Clinical Drug Candidates in Redox Medicine. Antioxid Redox Signal. 23:1113-29.

21. Dikalov SI, Nazarewicz RR (2013) Angiotensin II-induced production of mitochondrial reactive oxygen species: potential mechanisms and relevance for cardiovascular disease. Antioxid Redox Signal. 19:1085-94.

22. Doenst T, Nguyen TD, Abel ED (2013) Cardiac metabolism in heart failure: implications beyond ATP production. Circ Res. 113:709-24.

23. Dooley CT, Dore TM, Hanson GT, Jackson WC, Remington SJ, Tsien RY (2004) Imaging dynamic redox changes in mammalian cells with green fluorescent protein indicators. J Biol Chem. 279:22284-93.

24. Elahi MM, Kong YX, Matata BM (2009) Oxidative stress as a mediator of cardiovascular disease. Oxid Med Cell Longev. 2:259-69.

25. Eschenhagen T, Fink C, Remmers U, Scholz H, Wattchow J, Weil J, Zimmermann W, Dohmen HH, Schafer H, Bishopric N, Wakatsuki T, Elson EL (1997) Three-dimensional reconstitution of embryonic cardiomyocytes in a collagen matrix: a new heart muscle model system. FASEB journal : official publication of the Federation of American Societies for Experimental Biology. 11:683-94. 
26. Ezerina D, Morgan B, Dick TP (2014) Imaging dynamic redox processes with genetically encoded probes. J Mol Cell Cardiol. 73:43-9.

27. Farmakis D, Alvarez J, Gal TB, Brito D, Fedele F, Fonseca C, Gordon AC, Gotsman I, Grossini E, Guarracino F, Harjola VP, Hellman Y, Heunks L, Ivancan V, Karavidas A, Kivikko M, Lomivorotov V, Longrois D, Masip J, Metra M, Morelli A, Nikolaou M, Papp Z, Parkhomenko A, Poelzl G, Pollesello P, Ravn HB, Rex S, Riha H, Ricksten SE, Schwinger RH, Vrtovec B, Yilmaz MB, Zielinska M, Parissis J (2016) Levosimendan beyond inotropy and acute heart failure: Evidence of pleiotropic effects on the heart and other organs: An expert panel position paper. Int $J$ Cardiol. 222:303-12.

28. Fearon IM, Palmer AC, Balmforth AJ, Ball SG, Varadi G, Peers C (1999) Modulation of recombinant human cardiac L-type $\mathrm{Ca} 2+$ channel alpha1C subunits by redox agents and hypoxia. J Physiol. 514 ( Pt 3):629-37.

29. Forkink M, Smeitink JA, Brock R, Willems PH, Koopman WJ (2010) Detection and manipulation of mitochondrial reactive oxygen species in mammalian cells. Biochim Biophys Acta. 1797:1034-44.

30. Fujikawa Y, Roma LP, Sobotta MC, Rose AJ, Diaz MB, Locatelli G, Breckwoldt MO, Misgeld T, Kerschensteiner M, Herzig S, Muller-Decker K, Dick TP (2016) Mouse redox histology using genetically encoded probes. Sci Signal. 9:rs1.

31. Giles GI (2006) The redox regulation of thiol dependent signaling pathways in cancer. Curr Pharm Des. 12:4427-43.

32. Giordano FJ (2005) Oxygen, oxidative stress, hypoxia, and heart failure. J Clin Invest. 115:500-8.

33. Giovarelli M, Musiani P, Modesti A, Dellabona P, Casorati G, Allione A, Consalvo M, Cavallo F, di Pierro F, De Giovanni C, et al. (1995) Local release of IL10 by transfected mouse mammary adenocarcinoma cells does not suppress but 
enhances antitumor reaction and elicits a strong cytotoxic lymphocyte and antibodydependent immune memory. J Immunol. 155:3112-23.

34. Griendling KK, Minieri CA, Ollerenshaw JD, Alexander RW (1994) Angiotensin II stimulates NADH and NADPH oxidase activity in cultured vascular smooth muscle cells. Circ Res. 74:1141-8.

35. Griendling KK, Touyz RM, Zweier JL, Dikalov S, Chilian W, Chen YR, Harrison DG, Bhatnagar A (2016) Measurement of Reactive Oxygen Species, Reactive Nitrogen Species, and Redox-Dependent Signaling in the Cardiovascular System: A Scientific Statement From the American Heart Association. Circ Res. 119:e39-75.

36. Gutscher M, Pauleau AL, Marty L, Brach T, Wabnitz GH, Samstag Y, Meyer AJ, Dick TP (2008) Real-time imaging of the intracellular glutathione redox potential. Nat Methods. 5:553-9.

37. Hafstad AD, Nabeebaccus AA, Shah AM (2013) Novel aspects of ROS signalling in heart failure. Basic Res Cardiol. 108:359.

38. Handy DE, Loscalzo J (2016) Responses to reductive stress in the cardiovascular system. Free Radic Biol Med.

39. Hanson GT, Aggeler R, Oglesbee D, Cannon M, Capaldi RA, Tsien RY, Remington SJ (2004) Investigating mitochondrial redox potential with redox-sensitive green fluorescent protein indicators. J Biol Chem. 279:13044-53.

40. Hasslacher J, Bijuklic K, Bertocchi C, Kountchev J, Bellmann R, Dunzendorfer $S$, Joannidis M (2011) Levosimendan inhibits release of reactive oxygen species in polymorphonuclear leukocytes in vitro and in patients with acute heart failure and septic shock: a prospective observational study. Crit Care. 15:R166.

41. Hirt MN, Hansen A, Eschenhagen T (2014) Cardiac tissue engineering: state of the art. Circ Res. 114:354-67. 
42. Howard CM, Baudino TA (2014) Dynamic cell-cell and cell-ECM interactions in the heart. J Mol Cell Cardiol. 70:19-26.

43. Hoyle NP, O'Neill JS (2015) Oxidation-reduction cycles of peroxiredoxin proteins and nontranscriptional aspects of timekeeping. Biochemistry. 54:184-93.

44. Hwang C, Sinskey AJ, Lodish HF (1992) Oxidized redox state of glutathione in the endoplasmic reticulum. Science. 257:1496-502.

45. Irion S, Luche H, Gadue P, Fehling HJ, Kennedy M, Keller G (2007) Identification and targeting of the ROSA26 locus in human embryonic stem cells. Nat Biotechnol. 25:1477-82.

46. Kang SW, Rhee SG, Chang TS, Jeong W, Choi MH (2005) 2-Cys peroxiredoxin function in intracellular signal transduction: therapeutic implications. Trends Mol Med. 11:571-8.

47. Kappos L, Gold R, Miller DH, Macmanus DG, Havrdova E, Limmroth V, Polman CH, Schmierer K, Yousry TA, Yang M, Eraksoy M, Meluzinova E, Rektor I, Dawson KT, Sandrock AW, O'Neill GN (2008) Efficacy and safety of oral fumarate in patients with relapsing-remitting multiple sclerosis: a multicentre, randomised, double-blind, placebo-controlled phase Ilb study. Lancet. 372:1463-72.

48. Kawakami M, Okabe E (1998) Superoxide anion radical-triggered Ca2+ release from cardiac sarcoplasmic reticulum through ryanodine receptor $\mathrm{Ca2+}$ channel. Mol Pharmacol. 53:497-503.

49. Kline ER, Bassit L, Hernandez-Santiago BI, Detorio MA, Liang B, Kleinhenz DJ, Walp ER, Dikalov S, Jones DP, Schinazi RF, Sutliff RL (2009) Long-term exposure to AZT, but not $\mathrm{d} 4 \mathrm{~T}$, increases endothelial cell oxidative stress and mitochondrial dysfunction. Cardiovasc Toxicol. 9:1-12.

50. Kohler JJ, Cucoranu I, Fields E, Green E, He S, Hoying A, Russ R, Abuin A, Johnson D, Hosseini SH, Raper CM, Lewis W (2009) Transgenic mitochondrial 
superoxide dismutase and mitochondrially targeted catalase prevent antiretroviralinduced oxidative stress and cardiomyopathy. Lab Invest. 89:782-90.

51. Kuroda J, Ago T, Matsushima S, Zhai P, Schneider MD, Sadoshima J (2010) NADPH oxidase 4 (Nox4) is a major source of oxidative stress in the failing heart. Proc Natl Acad Sci U S A. 107:15565-70.

52. Kwon SH, Pimentel DR, Remondino A, Sawyer DB, Colucci WS (2003) $\mathrm{H}(2) \mathrm{O}(2)$ regulates cardiac myocyte phenotype via concentration-dependent activation of distinct kinase pathways. J Mol Cell Cardiol. 35:615-21.

53. Lassegue B, San Martin A, Griendling KK (2012) Biochemistry, physiology, and pathophysiology of NADPH oxidases in the cardiovascular system. Circ Res. 110:1364-90.

54. Liu J, Hinkhouse MM, Sun W, Weydert CJ, Ritchie JM, Oberley LW, Cullen JJ (2004) Redox regulation of pancreatic cancer cell growth: role of glutathione peroxidase in the suppression of the malignant phenotype. Hum Gene Ther. 15:23950 .

55. Lukyanov KA, Belousov VV (2014) Genetically encoded fluorescent redox sensors. Biochim Biophys Acta. 1840:745-56.

56. MacKenna D, Summerour SR, Villarreal FJ (2000) Role of mechanical factors in modulating cardiac fibroblast function and extracellular matrix synthesis. Cardiovasc Res. 46:257-63.

57. Malinouski M, Zhou Y, Belousov VV, Hatfield DL, Gladyshev VN (2011) Hydrogen peroxide probes directed to different cellular compartments. PLoS One. 6:e14564.

58. Manabe I, Shindo T, Nagai R (2002) Gene expression in fibroblasts and fibrosis: involvement in cardiac hypertrophy. Circ Res. 91:1103-13. 
59. Mari M, Morales A, Colell A, Garcia-Ruiz C, Fernandez-Checa JC (2009) Mitochondrial glutathione, a key survival antioxidant. Antioxid Redox Signal. 11:2685700 .

60. Mari M, Colell A, Morales A, von Montfort C, Garcia-Ruiz C, Fernandez-Checa JC (2010) Redox control of liver function in health and disease. Antioxid Redox Signal. 12:1295-331.

61. Maulucci G, Labate V, Mele M, Panieri E, Arcovito G, Galeotti T, Ostergaard H, Winther JR, De Spirito M, Pani G (2008) High-resolution imaging of redox signaling in live cells through an oxidation-sensitive yellow fluorescent protein. Sci Signal. 1:pl3.

62. McCord JM, Keele BB, Jr., Fridovich I (1971) An enzyme-based theory of obligate anaerobiosis: the physiological function of superoxide dismutase. Proc Natl Acad Sci U S A. 68:1024-7.

63. McCord JM (1988) Free radicals and myocardial ischemia: overview and outlook. Free Radic Biol Med. 4:9-14.

64. Meijs MF, Asselbergs FW, Doevendans PA (2012) Omecamtiv mecarbil: a promising new drug in systolic heart failure. Eur J Heart Fail. 14:232-3.

65. Meyer AJ, Dick TP (2010) Fluorescent protein-based redox probes. Antioxid Redox Signal. 13:621-50.

66. Morgan B, Sobotta MC, Dick TP (2011) Measuring $\mathrm{E}(\mathrm{GSH})$ and $\mathrm{H} 2 \mathrm{O} 2$ with roGFP2-based redox probes. Free Radic Biol Med. 51:1943-51.

67. Munns SE, Lui JK, Arthur PG (2005) Mitochondrial hydrogen peroxide production alters oxygen consumption in an oxygen-concentration-dependent manner. Free Radic Biol Med. 38:1594-603. 
68. Mussolino C, Alzubi J, Fine EJ, Morbitzer R, Cradick TJ, Lahaye T, Bao G, Cathomen T (2014) TALENs facilitate targeted genome editing in human cells with high specificity and low cytotoxicity. Nucleic acids research. 42:6762-73.

69. Nagahara N, Matsumura T, Okamoto R, Kajihara $Y$ (2009) Protein cysteine modifications: (1) medical chemistry for proteomics. Curr Med Chem. 16:4419-44.

70. Nagy L, Pollesello P, Papp Z (2014) Inotropes and inodilators for acute heart failure: sarcomere active drugs in focus. J Cardiovasc Pharmacol. 64:199-208.

71. Nakamura K, Fushimi K, Kouchi H, Mihara K, Miyazaki M, Ohe T, Namba M (1998) Inhibitory effects of antioxidants on neonatal rat cardiac myocyte hypertrophy induced by tumor necrosis factor-alpha and angiotensin II. Circulation. 98:794-9.

72. Ostergaard H, Henriksen A, Hansen FG, Winther JR (2001) Shedding light on disulfide bond formation: engineering a redox switch in green fluorescent protein. EMBO J. 20:5853-62.

73. Ostergaard H, Tachibana C, Winther JR (2004) Monitoring disulfide bond formation in the eukaryotic cytosol. J Cell Biol. 166:337-45.

74. Parissis JT, Farmakis D, Nieminen M (2007) Classical inotropes and new cardiac enhancers. Heart Fail Rev. 12:149-56.

75. Parissis JT, Andreadou I, Bistola V, Paraskevaidis I, Filippatos G, Kremastinos DT (2008) Novel biologic mechanisms of levosimendan and its effect on the failing heart. Expert Opin Investig Drugs. 17:1143-50.

76. Pollesello P, Papp Z, Papp JG (2016) Calcium sensitizers: What have we learned over the last 25 years? Int J Cardiol. 203:543-8.

77. Powis G, Mustacich D, Coon A (2000) The role of the redox protein thioredoxin in cell growth and cancer. Free Radic Biol Med. 29:312-22. 
78. Powis G, Montfort WR (2001) Properties and biological activities of thioredoxins. Annu Rev Biophys Biomol Struct. 30:421-55.

79. Raschi E, Vasina V, Ursino MG, Boriani G, Martoni A, De Ponti F (2010) Anticancer drugs and cardiotoxicity: Insights and perspectives in the era of targeted therapy. Pharmacol Ther. 125:196-218.

80. Reubinoff BE, Pera MF, Fong CY, Trounson A, Bongso A (2000) Embryonic stem cell lines from human blastocysts: somatic differentiation in vitro. Nat Biotechnol. 18:399-404.

81. Ribas V, Garcia-Ruiz C, Fernandez-Checa JC (2014) Glutathione and mitochondria. Front Pharmacol. 5:151.

82. Riquier S, Breton J, Abbas K, Cornu D, Bouton C, Drapier JC (2014) Peroxiredoxin post-translational modifications by redox messengers. Redox Biol. 2:777-85.

83. Sabri A, Byron KL, Samarel AM, Bell J, Lucchesi PA (1998) Hydrogen peroxide activates mitogen-activated protein kinases and $\mathrm{Na}+-\mathrm{H}+$ exchange in neonatal rat cardiac myocytes. Circ Res. 82:1053-62.

84. Sabri A, Hughie HH, Lucchesi PA (2003) Regulation of hypertrophic and apoptotic signaling pathways by reactive oxygen species in cardiac myocytes. Antioxid Redox Signal. 5:731-40.

85. Sahin AS, Gormus N, Duman A (2007) Preconditioning with levosimendan prevents contractile dysfunction due to $\mathrm{H} 2 \mathrm{O} 2$-induced oxidative stress in human myocardium. J Cardiovasc Pharmacol. 50:419-23.

86. Sano M, Fukuda K, Sato T, Kawaguchi H, Suematsu M, Matsuda S, Koyasu S, Matsui H, Yamauchi-Takihara K, Harada M, Saito Y, Ogawa S (2001) ERK and p38 MAPK, but not NF-kappaB, are critically involved in reactive oxygen speciesmediated induction of IL-6 by angiotensin II in cardiac fibroblasts. Circ Res. 89:661-9. 
87. Santos CX, Anilkumar N, Zhang M, Brewer AC, Shah AM (2011) Redox signaling in cardiac myocytes. Free Radic Biol Med. 50:777-93.

88. Santos CX, Raza S, Shah AM (2016) Redox signaling in the cardiomyocyte: From physiology to failure. Int J Biochem Cell Biol. 74:145-51.

89. Sarsour EH, Kumar MG, Chaudhuri L, Kalen AL, Goswami PC (2009) Redox control of the cell cycle in health and disease. Antioxid Redox Signal. 11:2985-3011.

90. Sawyer DB, Siwik DA, Xiao L, Pimentel DR, Singh K, Colucci WS (2002) Role of oxidative stress in myocardial hypertrophy and failure. J Mol Cell Cardiol. 34:37988.

91. Sawyer DB, Peng X, Chen B, Pentassuglia L, Lim CC (2010) Mechanisms of anthracycline cardiac injury: can we identify strategies for cardioprotection? Prog Cardiovasc Dis. 53:105-13.

92. Schafer FQ, Buettner GR (2001) Redox environment of the cell as viewed through the redox state of the glutathione disulfide/glutathione couple. Free Radic Biol Med. 30:1191-212.

93. Sies $H$ (2017) Hydrogen peroxide as a central redox signaling molecule in physiological oxidative stress: Oxidative eustress. Redox Biol. 11:613-619.

94. Sirker A, Zhang M, Shah AM (2011) NADPH oxidases in cardiovascular disease: insights from in vivo models and clinical studies. Basic Res Cardiol. 106:735-47.

95. Siwik DA, Pagano PJ, Colucci WS (2001) Oxidative stress regulates collagen synthesis and matrix metalloproteinase activity in cardiac fibroblasts. Am J Physiol Cell Physiol. 280:C53-60.

96. Sobotta MC, Barata AG, Schmidt U, Mueller S, Millonig G, Dick TP (2013) Exposing cells to $\mathrm{H} 2 \mathrm{O} 2$ : a quantitative comparison between continuous low-dose and one-time high-dose treatments. Free Radic Biol Med. 60:325-35. 
97. Soong PL, Tiburcy M, Zimmermann W-H (2012) Cardiac Differentiation of Human Embryonic Stem Cells and their Assembly into Engineered Heart Muscle.

98. Sorescu D, Griendling KK (2002) Reactive oxygen species, mitochondria, and $\mathrm{NAD}(\mathrm{P}) \mathrm{H}$ oxidases in the development and progression of heart failure. Congest Heart Fail. 8:132-40.

99. Souders CA, Bowers SL, Baudino TA (2009) Cardiac fibroblast: the renaissance cell. Circ Res. 105:1164-76.

100. Spinale FG (2002) Bioactive peptide signaling within the myocardial interstitium and the matrix metalloproteinases. Circ Res. 91:1082-4.

101. Swain L, Kesemeyer A, Meyer-Roxlau S, Vettel C, Zieseniss A, Guntsch A, Jatho A, Becker A, Nanadikar MS, Morgan B, Dennerlein S, Shah AM, El-Armouche A, Nikolaev VO, Katschinski DM (2016) Redox Imaging Using Cardiac MyocyteSpecific Transgenic Biosensor Mice. Circ Res. 119:1004-1016.

102. Takimoto E, Kass DA (2007) Role of oxidative stress in cardiac hypertrophy and remodeling. Hypertension. 49:241-8.

103. Teerlink JR (2009) A novel approach to improve cardiac performance: cardiac myosin activators. Heart Fail Rev. 14:289-98.

104. Tew KD, Townsend DM (2011) Redox platforms in cancer drug discovery and development. Curr Opin Chem Biol. 15:156-61.

105. Tian $\mathrm{Y}$, Morrisey EE (2012) Importance of myocyte-nonmyocyte interactions in cardiac development and disease. Circ Res. 110:1023-34.

106. Tiburcy M, Didie M, Boy O, Christalla P, Doker S, Naito H, Karikkineth BC, ElArmouche A, Grimm M, Nose M, Eschenhagen T, Zieseniss A, Katschinski DM, Hamdani N, Linke WA, Yin X, Mayr M, Zimmermann WH (2011) Terminal Differentiation, Advanced Organotypic Maturation, and Modeling of Hypertrophic Growth in Engineered Heart Tissue. Circulation Research. 109:1105-1114. 
107. Tiburcy M, Meyer T, Soong PL, Zimmermann WH (2014) Collagen-based engineered heart muscle. Methods Mol Biol. 1181:167-76.

108. Tiburcy M, Zimmermann WH (2014) Modeling myocardial growth and hypertrophy in engineered heart muscle. Trends Cardiovasc Med. 24:7-13.

109. Tiburcy M, Hudson JE, Balfanz R, Schlick S, Meyer T, Chang Liao ML, Levent E, Raad F, Zeidler S, Wingender E, Riegler J, Wang M, Gold JD, Kehat I, Wettwer E, Ravens U, Dierickx P, van Laake LW, Goumans MJ, Khadjeh S, Toischer K, Hasenfuss G, Couture LA, Unger A, Linke WA, Araki T, Neel B, Keller K, Gepstein L, Wu JC, Zimmermann WH (2017) Defined Engineered Human Myocardium with Advanced Maturation for Applications in Heart Failure Modelling and Repair. Circulation. accepted.

110. Tirziu D, Giordano FJ, Simons M (2010) Cell communications in the heart. Circulation. 122:928-37.

111. Tohyama S, Hattori F, Sano M, Hishiki T, Nagahata $Y$, Matsuura T, Hashimoto H, Suzuki T, Yamashita H, Satoh Y, Egashira T, Seki T, Muraoka N, Yamakawa H, Ohgino Y, Tanaka T, Yoichi M, Yuasa S, Murata M, Suematsu M, Fukuda K (2013) Distinct metabolic flow enables large-scale purification of mouse and human pluripotent stem cell-derived cardiomyocytes. Cell Stem Cell. 12:127-37.

112. Tomasek JJ, Gabbiani G, Hinz B, Chaponnier C, Brown RA (2002) Myofibroblasts and mechano-regulation of connective tissue remodelling. Nat Rev Mol Cell Biol. 3:349-63.

113. Tsutsui H, Kinugawa $S$, Matsushima $S$ (2011) Oxidative stress and heart failure. Am J Physiol Heart Circ Physiol. 301:H2181-90.

114. Vazquez-Medina JP, Popovich I, Thorwald MA, Viscarra JA, Rodriguez R, Sonanez-Organis JG, Lam L, Peti-Peterdi J, Nakano D, Nishiyama A, Ortiz RM (2013) Angiotensin receptor-mediated oxidative stress is associated with impaired 
cardiac redox signaling and mitochondrial function in insulin-resistant rats. $A m J$ Physiol Heart Circ Physiol. 305:H599-607.

115. Veal EA, Day AM, Morgan BA (2007) Hydrogen peroxide sensing and signaling. Mol Cell. 26:1-14.

116. von Harsdorf R, Li PF, Dietz R (1999) Signaling pathways in reactive oxygen species-induced cardiomyocyte apoptosis. Circulation. 99:2934-41.

117. Watson WH, Chen Y, Jones DP (2003) Redox state of glutathione and thioredoxin in differentiation and apoptosis. Biofactors. 17:307-14.

118. Werns SW, Lucchesi BR (1989) Myocardial ischemia and reperfusion: the role of oxygen radicals in tissue injury. Cardiovasc Drugs Ther. 2:761-9.

119. Weydert CJ, Cullen JJ (2010) Measurement of superoxide dismutase, catalase and glutathione peroxidase in cultured cells and tissue. Nat Protoc. 5:51-66.

120. Wood ZA, Poole LB, Karplus PA (2003) Peroxiredoxin evolution and the regulation of hydrogen peroxide signaling. Science. 300:650-3.

121. Xu KY, Zweier JL, Becker LC (1997) Hydroxyl radical inhibits sarcoplasmic reticulum $\mathrm{Ca}(2+)$-ATPase function by direct attack on the ATP binding site. Circ Res. 80:76-81.

122. Yoshioka $\mathrm{J}$ (2015) Thioredoxin superfamily and its effects on cardiac physiology and pathology. Compr Physiol. 5:513-30.

123. Zhang Y, Tocchetti CG, Krieg T, Moens AL (2012) Oxidative and nitrosative stress in the maintenance of myocardial function. Free Radic Biol Med. 53:1531-40.

124. Zhang Y, Martin SG (2014) Redox proteins and radiotherapy. Clin Oncol ( $R$ Coll Radiol). 26:289-300. 
125. Zima AV, Blatter LA (2006) Redox regulation of cardiac calcium channels and transporters. Cardiovasc Res. 71:310-21.

126. Zimmermann WH, Fink C, Kralisch D, Remmers U, Weil J, Eschenhagen $T$ (2000) Three-dimensional engineered heart tissue from neonatal rat cardiac myocytes. Biotechnology and bioengineering. 68:106-14.

127. Zimmermann $\mathrm{WH}$, Schneiderbanger $\mathrm{K}$, Schubert $\mathrm{P}$, Didie M, Munzel F, Heubach JF, Kostin S, Neuhuber WL, Eschenhagen T (2002) Tissue engineering of a differentiated cardiac muscle construct. Circ Res. 90:223-30. 